\title{
Test Plan for BWID Phase 2 Electric Arc Melter Vitrification Tests
}

\author{
N. R. Soelberg \\ P. C. Turner \\ L. L. Oden \\ G. L. Anderson
}

Published October 1994

Idaho National Engineering Laboratory Lockheed Idaho Technologies Company

Idaho Falls, Idaho 83415 


\section{DISCLAIMER}

This report was prepared as an account of work sponsored by an agency of the United States Government. Neither the United States Government nor any agency thereof, nor any of their employees, make any warranty, express or implied, or assumes any legal liability or responsibility for the accuracy, completeness, or usefulness of any information, apparatus, product, or process disclosed, or represents that its use would not infringe privately owned rights. Reference herein to any specific commercial product, process, or service by trade name, trademark, manufacturer, or otherwise does not necessarily constitute or imply its endorsement, recommendation, or favoring by the United States Government or any agency thereof. The views and opinions of authors expressed herein do not necessarily state or reflect those of the United States Government or any agency thereof. 


\section{DISCLAIMER}

Portions of this document may be illegible in electronic image products. Images are produced from the best available original document. 


\section{Test Plan for BWID Phase 2 Electric Arc Melter Vitrification Tests}

INEL-94/0004

Prepared by

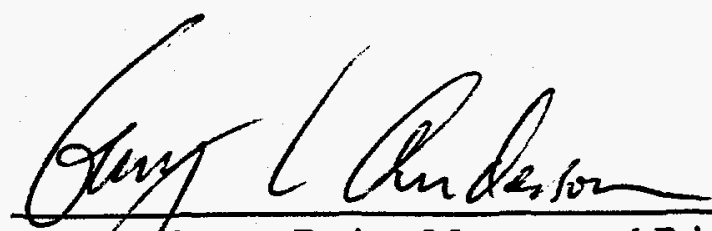

G. L. Axderson, Project Manager and Principal Investigator

Waśte/Technology Development

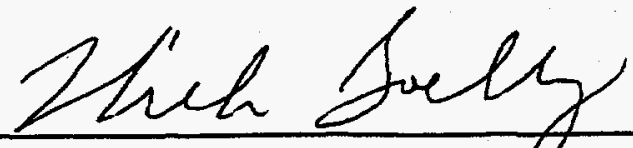

N. R. Soelberg, Co-Principal Investigator

Chemical and Environmental Engineering

Reviewed and approved by

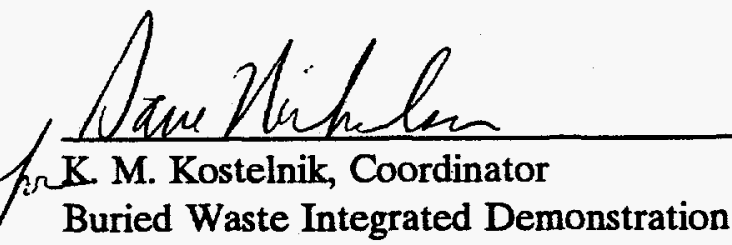




\section{ABSTRACT}

This test plan describes the Buried Waste Integrated Demonstration (BWID), Phase 2, electric arc melter, waste treatment evaluation tests to be performed at the U.S. Bureau of Mines (USBM) Albany Research Center. The BWID Arc Melter Vitrification Project is being conducted to evaluate and demonstrate existing industrial arc melter technology for thermally treating mixed transuranic-contaminated wastes and soils. Phase 1 baseline tests, performed during fiscal year 1993 at the USBM, were conducted on waste feeds representing incinerated buried mixed wastes and soils. In Phase 2, surrogate feeds will be processed that represent actual as-retrieved buried wastes from the Idaho National Engineering Laboratory's Subsurface Disposal Area at the Radioactive Waste Management Complex. 


\section{SUMMARY}

This test plan describes the Buried Waste Integrated Demonstration (BWID) Phase 2 electric arc melter tests to be performed at the U.S. Bureau of Mines (USBM) Albany Research Center (ALRC). This Arc Melter Vitrification Project is being conducted to evaluate and demonstrate application of industrial arc melter technology for thermally treating mixed transuranic (TRU) contaminated wastes and soils. Phase 1 (baseline) tests were performed during fiscal year (FY) 1993. The Phase 1 treatment processing tests were successfully performed using the existing pilot-scale USBM arc melter waste treatment system on surrogate feed mixtures prepared for "as-incinerated" buried wastes mixed with soil. Surrogates of as-incinerated wastes mixed with soil were used under the premise that in actual treatment practice, an arc melter may be used to vitrify ash generated from previous incineration of the buried waste.

In Phase 2, surrogate feeds will be processed that represent actual as-retrieved buried wastes from the Idaho National Engineering Laboratory (INEL) Subsurface Disposal Area (SDA) at the Radioactive Waste Management Complex (RWMC). These "as retrieved" buried waste feed materials contain substantial fractions of combustible organics. Phase 2 testing will, therefore, evaluate the premise that the arc melter can be used directly for waste treatment without a prior incineration step. Testing of actual bottom ash from a utility refuse-derived furnace (RDF) will also be done during the Phase 2 test series, on behalf of Southern California Edison (SCE). The RDF bottom ash is being provided by SCE. Objectives of the Phase 2 tests include

1. Determine equipment capability to process (a) untreated buried wastestreams, with the ultimate objective to treat the BWID Nominal Standard Wastestream (60 wt\% RWMC soil, $15 \%$ metal, $10 \%$ solid combustibles, $9 \%$ absorbed organics, $4 \%$ hydroxide sludges, and $2 \%$ nitrate sludges) and (b) RDF bottom ash.

2. Evaluate the extent of thermal breakdown and partial oxidation (pyrolysis) in the melter of solid carbonaceous materials and absorbed liquid hydrocarbons in furnace feeds.

3. Determine the effects of and potential for oxidation control in the furnace using air injection through the feed tubes, addition of solid oxidizers in the feed, and/or injection of oxidizer by lancing.

4. Demonstrate complete thermal oxidation of arc furnace offgases and entrained soot in a close-coupled thermal oxidizer.

5. Demonstrate continuous feeding, slag tapping, and as-needed metals tapping capability.

6. Determine composition, homogeneity, and properties of the slag, offgas, and metal products.

7. Evaluate partitioning of a TRU surrogate (Ce), toxic metals, and other elements such as $\mathrm{C}, \mathrm{Cl}$, and $\mathrm{S}$ among the slag, metal, air pollution control system (APCS) solids, and offgas phases. 
8. Determine the effect of cold top (unmelted burden of feed material within the furnace) on furnace process operations, offgas composition, vaporization of metals in the feed, particulate carryover, and furnace performance parameters, with emphasis on reducing metals volatilization, minimizing particulate entrainment, minimizing offgas temperature, and maximizing thermal breakdown of hydrocarbons to simple, noncondensible species.

The main features of the Phase 2 test program include

- Modifications to the existing APCS to enable control of pyrolysis and combustion products and acid gases along with the existing particulate control.

- Preliminary shakedown tests designed to evaluate the furnace and new APCS performance.

- Four days of 24-hour continuous demonstration testing, including 1 day of processing the RDF bottom ash, and 3 days of processing buried waste surrogate mixtures.

- Measurements and sample collection will be performed during the demonstration tests to ensure safe operation, characterize operation of the main system components (feed system, furnace, and APCS) and conduct mass balances to evaluate the fate of feed components such as toxic metals, structural metals, and a TRU surrogate. 


\section{CONTENTS}

ABSTRACT $\ldots \ldots \ldots \ldots \ldots \ldots \ldots \ldots \ldots \ldots \ldots \ldots \ldots \ldots \ldots \ldots \ldots \ldots \ldots \ldots \ldots \ldots$, iii

SUMMARY $\ldots \ldots \ldots \ldots \ldots \ldots \ldots \ldots \ldots \ldots \ldots \ldots \ldots \ldots \ldots \ldots \ldots \ldots \ldots \ldots \ldots \ldots, \quad v$

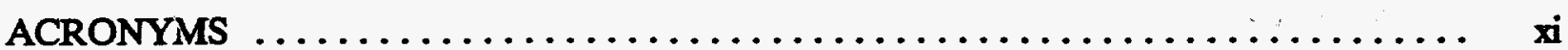

1. INTRODUCTION $\ldots \ldots \ldots \ldots \ldots \ldots \ldots \ldots \ldots \ldots \ldots \ldots \ldots \ldots \ldots \ldots \ldots \ldots \ldots, 1-1$

1.1 Technology Description and Background $\ldots \ldots \ldots \ldots \ldots \ldots \ldots \ldots \ldots, 1-1$

1.2 Scope $\ldots \ldots \ldots \ldots \ldots \ldots \ldots \ldots \ldots \ldots \ldots \ldots \ldots \ldots \ldots \ldots \ldots, 1-2$

1.3 Objectives $\ldots \ldots \ldots \ldots \ldots \ldots \ldots \ldots \ldots \ldots \ldots \ldots \ldots \ldots \ldots \ldots \ldots \ldots \ldots \ldots \ldots, 1-2$

1.4 Technology Agreement $\ldots \ldots \ldots \ldots \ldots \ldots \ldots \ldots \ldots \ldots \ldots \ldots \ldots, \quad 1-3$

2. ORGANIZATION AND RESPONSIBILITIES $\ldots \ldots \ldots \ldots \ldots \ldots \ldots \ldots \ldots .2-1$

2.1 Personnel Support Requirements, Qualifications, and Training $\ldots \ldots \ldots \ldots$ 2-1

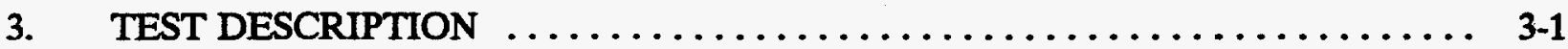

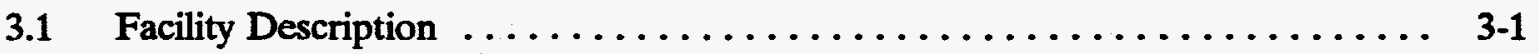

3.1.1 Furnace, Feed System, and Power Supply $\ldots \ldots \ldots \ldots \ldots \ldots \ldots$ 3-1



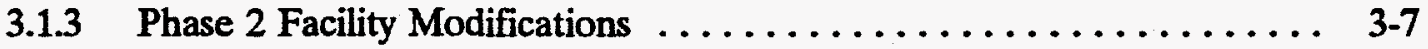

3.1.4 Sample Locations and Sample Ports .................. 3-7

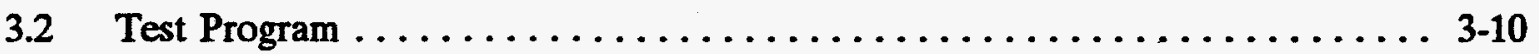

3.2.1 Feed Mixtures $\ldots \ldots \ldots \ldots \ldots \ldots \ldots \ldots \ldots \ldots \ldots \ldots \ldots \ldots \ldots, 14$

3.2.2 Test Uncertainties $\ldots \ldots \ldots \ldots \ldots \ldots \ldots \ldots \ldots \ldots \ldots \ldots \ldots \ldots \ldots \ldots \ldots, 14$

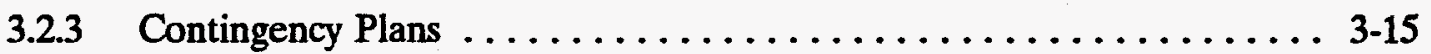

4. SEQUENCE OF ACTIVITIES $\ldots \ldots \ldots \ldots \ldots \ldots \ldots \ldots \ldots \ldots \ldots \ldots \ldots \ldots \ldots \ldots \ldots$ 4-1

5. MEASUREMENTS AND SAMPLE COLLECTION $\ldots \ldots \ldots \ldots \ldots \ldots \ldots$. 5 -1

5.1 Melter Furnace Process Continuous Monitoring $\ldots \ldots \ldots \ldots \ldots \ldots \ldots$ 5-1

5.2 Process Sample Collection $\ldots \ldots \ldots \ldots \ldots \ldots \ldots \ldots \ldots \ldots \ldots \ldots$ 5-1

5.3 Gaseous and Particulate Measurements and Sample Collection ......... 5-1 
5.3.1 High-temperature Measurements . .................. 5-9

5.3.2 Continuous Gaseous Emissions Monitoring Procedures $\ldots \ldots \ldots \ldots$ 5-13

5.3.3 Gas Chromatography Procedures ................... 5-14

5.3.4 Metals and Particulate Sampling Procedures ............... 5-14

5.3.5 Offgas Sampling Team $\ldots \ldots \ldots \ldots \ldots \ldots \ldots \ldots \ldots \ldots \ldots, 5-15$

6. DOCUMENT CONTROL $\ldots \ldots \ldots \ldots \ldots \ldots \ldots \ldots \ldots \ldots \ldots \ldots \ldots \ldots \ldots \ldots \ldots$

6.1 Data Documentation, Control, and Storage $\ldots \ldots \ldots \ldots \ldots \ldots \ldots \ldots$ 6-1

6.2 Test Plan Change Control $\ldots \ldots \ldots \ldots \ldots \ldots \ldots \ldots \ldots \ldots \ldots \ldots, 6,1$

6.3 Document Control for Other Documents $\ldots \ldots \ldots \ldots \ldots \ldots \ldots \ldots \ldots, 6-2$

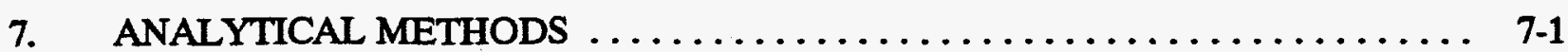

8. DATA REDUCTION, VALIDATION, AND VERIFICATION $\ldots \ldots \ldots \ldots \ldots \ldots$ 8-1

$8.1 \quad$ Data Reduction Scheme $\ldots \ldots \ldots \ldots \ldots \ldots \ldots \ldots \ldots \ldots \ldots \ldots, 8,1$

8.2 Data Validation $\ldots \ldots \ldots \ldots \ldots \ldots \ldots \ldots \ldots \ldots \ldots \ldots \ldots \ldots, \quad 8-2$

8.3 Data Validation Responsibilities $\ldots \ldots \ldots \ldots \ldots \ldots \ldots \ldots \ldots \ldots \ldots, 8.3$

8.4 Acceptance Criteria for Test Data $\ldots \ldots \ldots \ldots \ldots \ldots \ldots \ldots \ldots \ldots$ 8-3

9. QUALITY ASSURANCE $\ldots \ldots \ldots \ldots \ldots \ldots \ldots \ldots \ldots \ldots \ldots \ldots \ldots \ldots \ldots \ldots \ldots \ldots$

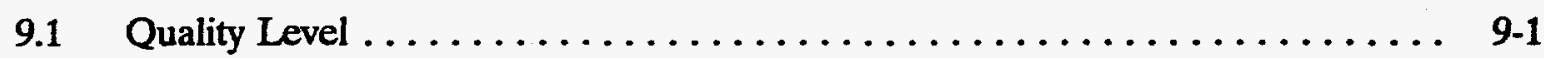

9.1.1 Data Quality Objectives ..................... 9-2

9.2 Quality Control Methods $\ldots \ldots \ldots \ldots \ldots \ldots \ldots \ldots \ldots \ldots \ldots$ 9.4

9.3 Routine Procedures $\ldots \ldots \ldots \ldots \ldots \ldots \ldots \ldots \ldots \ldots \ldots \ldots \ldots \ldots .9 .7$

9.4 Internal Audit System $\ldots \ldots \ldots \ldots \ldots \ldots \ldots \ldots \ldots \ldots \ldots \ldots \ldots \ldots$

10. EQUIPMENT AND INSTRUMENTS $\ldots \ldots \ldots \ldots \ldots \ldots \ldots \ldots \ldots \ldots \ldots \ldots \ldots \ldots$

10.1 Range, Accuracy, and Tolerance Levels $\ldots \ldots \ldots \ldots \ldots \ldots \ldots \ldots \ldots \ldots \ldots \ldots \ldots$

10.2 Calibration and Standardization Procedures $\ldots \ldots \ldots \ldots \ldots \ldots \ldots \ldots \ldots \ldots \ldots \ldots$

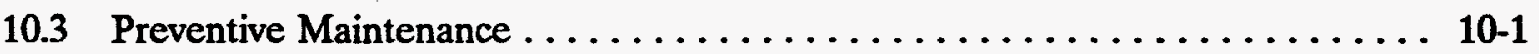

11. SUPPLIES, UTILITIES, AND FACILITIES $\ldots \ldots \ldots \ldots \ldots \ldots \ldots \ldots \ldots \ldots \ldots$ 
13. RESIDUALS MANAGEMENT

14. REFERENCES .

Appendix A-BWID Nominal Standard Waste Mixture Tables

Appendix B-Calculated Proximate Analysis for the BWID Phase 2 Feed Mixtures

Appendix C-Estimated Offgas Compositions and Flowrates

Appendix D-Estimated Elemental Compositions of the Surrogate Mixture Components . .

Appendix E-Reconciliation of the Test Plan to the BWID Technology Test Plan Guidance Document

\section{FIGURES}

3-1. Schematic of the feed system, furnace, and thermal oxidizer $\ldots \ldots \ldots \ldots \ldots \ldots$

3-2. Schematic of the offgas system from the furnace to the baghouse $\ldots \ldots \ldots \ldots$

3-3. Schematic of the offgas system downstream of the baghouse $\ldots \ldots \ldots \ldots \ldots$

3-4. Schematic of the arc melter furnace $\ldots \ldots \ldots \ldots \ldots \ldots \ldots \ldots \ldots \ldots$

5-1. Seal for leak-tight ports during sampling $\ldots \ldots \ldots \ldots \ldots \ldots \ldots \ldots \ldots \ldots \ldots$

5-2. Schematic of typical water-cooled suction pyrometer probe $\ldots \ldots \ldots \ldots \ldots \ldots \ldots$

5-3. CEMS bypass for on the pyrometer suction system $\ldots \ldots \ldots \ldots \ldots \ldots \ldots \ldots$

\section{TABLES}

3-1. Furnace and APCS sample locations and ports $\ldots \ldots \ldots \ldots \ldots \ldots \ldots \ldots$

3-2. Test matrix for the demonstration tests $\ldots \ldots \ldots \ldots \ldots \ldots \ldots \ldots \ldots \ldots \ldots \ldots$

3-3. Sample collection and measurement matrix for the demonstration tests $\ldots \ldots \ldots . .3$ 3-12

5-1. ALRC melter process monitoring summary $\ldots \ldots \ldots \ldots \ldots \ldots \ldots \ldots \ldots \ldots$

5-2. Sample collection summary $\ldots \ldots \ldots \ldots \ldots \ldots \ldots \ldots \ldots \ldots \ldots \ldots \ldots$

5-3. Process sample handling summary $\ldots \ldots \ldots \ldots \ldots \ldots \ldots \ldots \ldots \ldots \ldots$ 
5-4. Gaseous and particulate measurement and sampling procedures

7-1. Sample analysis $\operatorname{matrix} \ldots \ldots \ldots \ldots \ldots \ldots \ldots \ldots \ldots \ldots \ldots \ldots \ldots \ldots \ldots \ldots$

7-2. Sample analysis procedures $\ldots \ldots \ldots \ldots \ldots \ldots \ldots \ldots \ldots \ldots \ldots \ldots \ldots \ldots$

8-1. Offgas equipment calibrations $\ldots \ldots \ldots \ldots \ldots \ldots \ldots \ldots \ldots \ldots \ldots \ldots \ldots \ldots \ldots$

8-2. Summary of acceptance criteria for offgas sampling $\ldots \ldots \ldots \ldots \ldots \ldots \ldots \ldots$

8-3. Acceptance criteria for offgas continuous monitoring equipment $\ldots \ldots \ldots \ldots$

9-1. Data quality objectives for critical measurements $\ldots \ldots \ldots \ldots \ldots \ldots \ldots \ldots$

9-2. Internal QC checks $\ldots \ldots \ldots \ldots \ldots \ldots \ldots \ldots \ldots \ldots \ldots \ldots \ldots \ldots \ldots \ldots$

9-3. Internal QC sample matrix for particulate and metals measurements for the MMT samples and process samples $\ldots \ldots \ldots \ldots \ldots \ldots \ldots \ldots$

9-4. Internal audits and QA checks $\ldots \ldots \ldots \ldots \ldots \ldots \ldots \ldots \ldots \ldots \ldots \ldots$ 


\section{ACRONYMS}

\begin{tabular}{|c|c|}
\hline $\mathrm{AC}$ & Alternating current \\
\hline ALRC & Albany Research Center \\
\hline APCS & Air Pollution Control System \\
\hline ASME & American Society of Mechanical Engineers \\
\hline BWID & Buried Waste Integrated Demonstration \\
\hline CEMS & Continuous emissions monitoring system \\
\hline CVAAS & Cold-vapor atomic absorption spectroscopy \\
\hline DAS & Data Acquisition System \\
\hline DC & Direct current \\
\hline EPA & Environmental Protection Agency \\
\hline FY & Fiscal year \\
\hline GFAAS & Graphite furnace atomic absorption spectroscopy \\
\hline HEPA & High efficiency particulate air \\
\hline ICAP & Ion-coupled argon plasma spectroscopy \\
\hline ID & Inside diameter \\
\hline IEB & Iron-enriched basalt \\
\hline INEL & Idaho National Engineering Laboratory \\
\hline LITCO & Lockheed Idaho Technologies Company \\
\hline MMT & Multiple metal train \\
\hline PPE & Personnel protective equipment \\
\hline QA & Quality assurance \\
\hline QC & Quality control \\
\hline RDF & Refuse Derived Furnace \\
\hline
\end{tabular}


RWMC Radioactive Waste Management Complex

SCE Southern California Edison

SDA Subsurface Disposal Area

SOP Standard Operating Procedure

TCLP Toxicity characteristic leachability procedure

TRU Transuranic

TTP Technical Task Plan

USBM U.S. Bureau of Mines 


\section{Test Plan for BWID Phase 2 Electric Arc Melter Vitrification Tests}

\section{INTRODUCTION}

Substantial quantities (approximately 2.3 million cubic feet) of low-level, transuranic (TRU) and mixed wastes are buried at the Idaho National Engineering Laboratory's (INEL's) Radioactive Waste Management Complex (RWMC) Subsurface Disposal Area (SDA) ${ }^{1}$ The amount of associated, potentially contaminated soil is estimated at 8 million cubic feet. By volume, combustible materials (wood, paper, plastics, cloth, rags, rubber, etc.) are the largest single category of waste, but there are also large amounts of cemented and uncemented organic and inorganic sludges, nitrate salts, metals, and other noncombustible, nonmetal materials (insulation, glass, soil, concrete, etc.). The wastes are not well characterized or segregated. These wastes and contaminated soils are expected to require treatment to remove or destroy hazardous organics and stabilize the radioactive and hazardous metal constituents. Treatment may also significantly homogenize the wastes and reduce the final disposal volume.

Various treatment technologies are being evaluated and demonstrated for potential application to buried wastes. $2,3,4$ The most desirable approach for long-term stabilization of the TRU elements is believed to be high-temperature melting of the waste materials to (a) destroy hazardous organic compounds and (b) dissolve the TRU elements and toxic metals, as oxides, into a slag. 5,6 Upon cooling, the slag will solidify into a durable amorphous glass or crystalline rock-like matrix, depending on composition and cooling rates. The slag can immobilize both the radionuclides and toxic metals in a homogeneous, long-lived waste form. Depending on the physical form of the feed materials, there can also be considerable volume reduction.

This test plan was prepared in accordance with the BWID technology test plan guidance document, ${ }^{7}$ but with some deviations which were pertinent to this specific test program. Several of the sections specified in the guidance document not relevant to this program and were excluded. Some other sections have been reorganized so the test plan is more directly focused on the technical test program.

\subsection{Technology Description and Background}

Industrial arc melter technology is well developed for high-temperature pyrometallurgical field applications $s^{6,7,8}$ that routinely process large volumes of heterogeneous ore materials similar to buried wastes and soils. Electric arc furnaces are available in a multitude of design configurations for various applications including three-phase alternating current $(\mathrm{AC})$ with three or more electrodes, singlephase $A C$ with two or more electrodes, and direct current (DC) with one electrode at the center and a return under the melt. Power levels up to $105 \mathrm{MW}$ have been built that are capable of processing up to $\mathbf{1 3 0}$ tons/h of ore material. Units such as those built for the processing of phosphorous have been well sealed to prevent influx of undesirable, oxidizing air and/or fugitive emissions. High processing temperatures are possible in the arc furnace. For example, melt processing of refractory oxides, such as magnesia, can achieve temperatures of $2,800^{\circ} \mathrm{C}$. 
Among arc melter processes, three-phase AC electric arc furnaces have been the most widely used for industrial applications and represent a reliable baseline arc melter technology. Most recently, DC powered configurations have re-emerged in the steel industry as an alternative configuration.

A small, industrial-scale, three-phase $\mathrm{AC}$ graphite electrode electric arc furnace located at the Albany Research Center (ALRC) of the United States Bureau of Mines (USBM) is being used for the demonstration tests. This furnace was originally constructed for experimental vitrification of municipal incinerator ash by a consortium organized by the American Society of Mechanical Engineers (ASME). ${ }^{8}$ After completion of the ASME tests, the INEL entered into an agreement with the USBM to modify the furnace and use it for testing and developing an arc melter process for vitrification of buried mixed wastes.

\subsection{Scope}

Unique features of this program include the demonstration of arc melter technology commonly used in industry, using a test facility that is amenable to process and design modifications and performing diagnostic and instrumental measurements. This work, funded by the U.S. Department of Energy's Office of Technology Development through the Buried Waste Integrated Demonstration (BWID), has been planned to proceed in phases. Phase 1, conducted in fiscal year (FY) 1993, was a series of baseline tests on existing equipment with surrogate wastestreams representing previously incinerated waste. In Phase 2, the processing of complex raw feeds materials, including mixtures containing combustible solids and organic solvents, reactive nitrates, and other types of wastes will be evaluated.

In Phase 3, proposed to be conducted based upon the results from Phase 2, more specific evaluation and process optimization will be performed. These technology evaluation tests will provide data needed for designing, building, and operating pilot-scale facilities for radioactive waste treatment testing by FY-97.

\subsection{Objectives}

The purpose of the BWID Arc Melter Vitrification Project is to evaluate and demonstrate the application of existing industrial arc melter processing technology to the treatment of buried/mixed wastes and soils. Top level objectives for all experimental phases include

- Evaluate and demonstrate application of industrial arc melter processing technology for thermal treatment of mixed TRU contaminated wastes and soils

- Obtain performance data for Environmental Restoration and Waste Management treatment systems design/selection

- Provide data on a large scale for use in developing/verifying analytical tools, models, and scaling relationships for melter system and process design evaluations 
- Identify technology gaps and development needs that require closure for deployment of the technology in a production process environment

- Define and evaluate potential process, equipment, and operations improvement modifications.

To meet these overall demonstration program objectives, the objectives of the Phase 2 test include

1. Determine equipment capability to process (a) untreated buried wastestreams, with the ultimate objective to treat the BWID Nominal Standard Wastestream (60 wt\% RWMC soil, $15 \%$ metal, $10 \%$ solid combustibles, $9 \%$ absorbed organics, $4 \%$ hydroxide sludges, and 2\% nitrate sludges) and (b) Refuse Derived Furnace (RDF) bottom ash

2. Evaluate the extent of thermal breakdown and partial oxidation (pyrolysis) in the arc furnace of solid carbonaceous materials and absorbed liquid hydrocarbons in furnace feeds

3. Determine the effects of and potential for oxidation control in the furnace utilizing air injection through the feed tubes, addition of solid oxidizers in the feed, and/or injection of oxidizer by lancing

4. Demonstrate complete thermal oxidation of furnace offgases and entrained soot in a closecoupled thermal oxidizer

5. Demonstrate continuous slag tapping and as-needed metals tapping capability

6. Determine composition, homogeneity, and properties of the slag, offgas, and metal products

7. Evaluate partitioning of a TRU surrogate (Ce), toxic metals, and other elements such as $\mathrm{C}, \mathrm{Cl}$, and $\mathrm{S}$ among the slag, metal, air pollution control system (APCS) solids, and offgas phases

8. Determine the effect of cold top (unmelted burden of feed material within the furnace) on furnace process operations, offgas composition, vaporization of metals in the feed, particulate carryover, and furnace performance parameters, with emphasis on reducing metals volatilization, minimizing particulate entrainment, minimizing offgas temperature, and maximizing thermal breakdown of hydrocarbons to simple, noncondensible species.

\subsection{Technology Agreement}

This test plan defines the Phase 2 test series of the BWID Arc Melter Vitrification Project in accordance with the scope of the Technical Task Plan (TTP) No. ID 132010, Revision 0. The technology agreement is based on TTP ID 132011. 


\section{ORGANIZATION AND RESPONSIBILITIES}

The testing will be performed by USBM ALRC with support from Lockheed Idaho Technologies Company (LITCO) consultants, and subcontractors. G. L. Anderson is the Arc Melter Vitrification Project Manager and a co-Principal Investigator. N. R. Soelberg is co-Principal Investigator and will also supervise the Offgas Sampling and Analysis Team during the demonstration tests. Dr. P. C. Turner is the Melter Facility Project Manager, and Dr. L. L. Oden is the Melter Operations Director. Activities and organizational responsibilities include

- $\quad$ APCS modifications: Designs-Enercon, ALRC, and LITCO; vendor equipment purchase (if needed)-LITCO; fabrication and installation-ALRC

- Sample port design and specification-LITCO, with approval and installation by ALRC

- Surrogate waste preparation-ALRC [40,000-1b RWMC soil shipped to ALRC by LITCO and acquisition of additives by ALRC, and shipment of RDF bottom ash to ALRC by Southern California Edison (SCE)]

- Melter test system and facility preparation and operation, process monitoring, process sample collection, and sample analysis-ALRC

- Offgas sampling and analysis, determination of sample analysis requirements, and quality assurance (QA)-ALRC, LITCO with subcontracted sampling team.

\subsection{Personnel Support Requirements, Qualifications, and Training}

ALRC personnel will direct the test operations and will perform the melter operation, process monitoring, and process sample collection during the planned tests. LTTCO personnel will assist the ALRC in test coordination and will direct offgas sampling and analysis. The offgas sampling and analysis will be conducted by ALRC, LITCO, and subcontracted stack testing personnel and equipment. Samples collected will be submitted to ALRC for sample analysis. Laboratory analysis will be performed by USBM at the ALRC laboratories and/or other appropriate laboratories.

ALRC has an experienced team of personnel to run the tests. The ALRC has extensive experience in arc furnace and metallurgical operations, including the design, construction, and operation of the melter facility. The same personnel who operated the melter system for the ASME municipal incinerator ash tests and the FY-93 BWID arc melter demonstration tests will operate the system for the Phase 2 tests. The ALRC analytical laboratory has experienced technicians who have used or pioneered all of the analytical procedures specified for use at the ALRC laboratory. Any analytical procedures that cannot be performed at the ALRC laboratory will be subcontracted to an outside laboratory experienced with the specified procedures and sample matrices. The subcontracted stack testing company will be required contractually to provide engineers and technicians for onsite sampling and analytical support who are experienced in the specific duties. 


\section{TEST DESCRIPTION}

\subsection{Facility Description}

The melter facility is the same system that was used in the Phase 1 tests, with a completely modified APCS. The APCS had to be revised to enable feed materials with significant amounts of organic material and halogens to be processed. These modifications, sample port installations, and a general description of the modified facility are included in this section. More detailed descriptions of the melter system are provided in the Phase 1 test plan ${ }^{9}$ and the Phase 1 data report. ${ }^{10}$

The melter system consists of a continuous feed system, furnace, APCS, and facility power, water, instrumentation, and controls. The feed system, furnace, and thermal oxidizer are shown in Figure 3-1. The APCS is shown in Figures 3-2 and 3-3.

\subsubsection{Furnace, Feed System, and Power Supply}

The furnace (Figure 3-4) is a stationary, refractory-lined, small industrial-scale, electric arc melting furnace that is sealed for atmosphere control. The furnace has three moveable 4-in. diameter solid graphite electrodes, automatic power, and electrode vertical positioning control. Power is supplied by a fully instrumented $800 \mathrm{kVA}$ three-phase supply. The electrodes are positioned at the apexes of an equilateral triangle $\mathbf{1 1 . 2 5}$ in. from electrode center to center. The electrodes and four water-cooled feed tubes extend through ports the furnace roof. The feed tubes, furnace roof and sidewalls, and copper slag tapping fixture are water cooled. Slag can be continuously tapped from the furnace; metal is tapped (and the hearth is emptied) through a hole in the bottom center of the hearth.

The furnace shell bottom is lined with 5 in. ( 2 courses) of chromic oxide-alumina bonded, 90 pct alumina super duty straight firebricks (Ruby) from Harbison-Walker Refractories. Five courses of ruby key bricks shape the hearth and form the sidewall up to the steel shelf that is $28 \mathrm{in}$. above the bottom and approximately 7 in. above the slag tap hole. One inch of dry phosphate bonds the silicon carbide ( $\mathrm{SiC}$ ) ramming mix (Norton Company) that was placed between the steel bottom and first course of ruby straights to improve heat transfer to the bottom. One inch of the ramming mix is also placed between the ruby keys and sidewall for heat transfer improvement. The upper sidewall is lined with 4.5-in. GM $70 \mathrm{DE}$ (70\% alumina) insulating fire brick from North American Refractories. Fiberfrax batting (Carborundum Company) 1 in. thick is placed between the sidewall and arch brick as added insulation. The hearth is rammed to 6 in. deep at the center with Ruby ramming mix from Harbison-Walker. The roof is lined with 4 in. of Narphos $85 P$ plastic refractory ( $85 \%$ alumina) from North American Refractories. The finished inside diameter of the furnace is about 46 in. at midheight. The capacity of the hearth is approximately $5 \mathrm{ft}^{3}$.

The metal tap hole is 1.5 in. in diameter. The copper, water-jacketed tapping fixture is 6 in. outside diameter and $7.5 \mathrm{in.}$ long, with a tap hole diameter of 5/8 in.

The carbon steel shell of the furnace is $65 \mathrm{in}$. high and tapers from $60 \mathrm{in}$. inside diameter (ID) at the bottom to 56-in. ID at the top. Three carbon steel straps ( 2 in. wide $\times 0.060$ in. thick) were welded to the inside bottom of the shell in a triangular array before placing the refractory lining in 


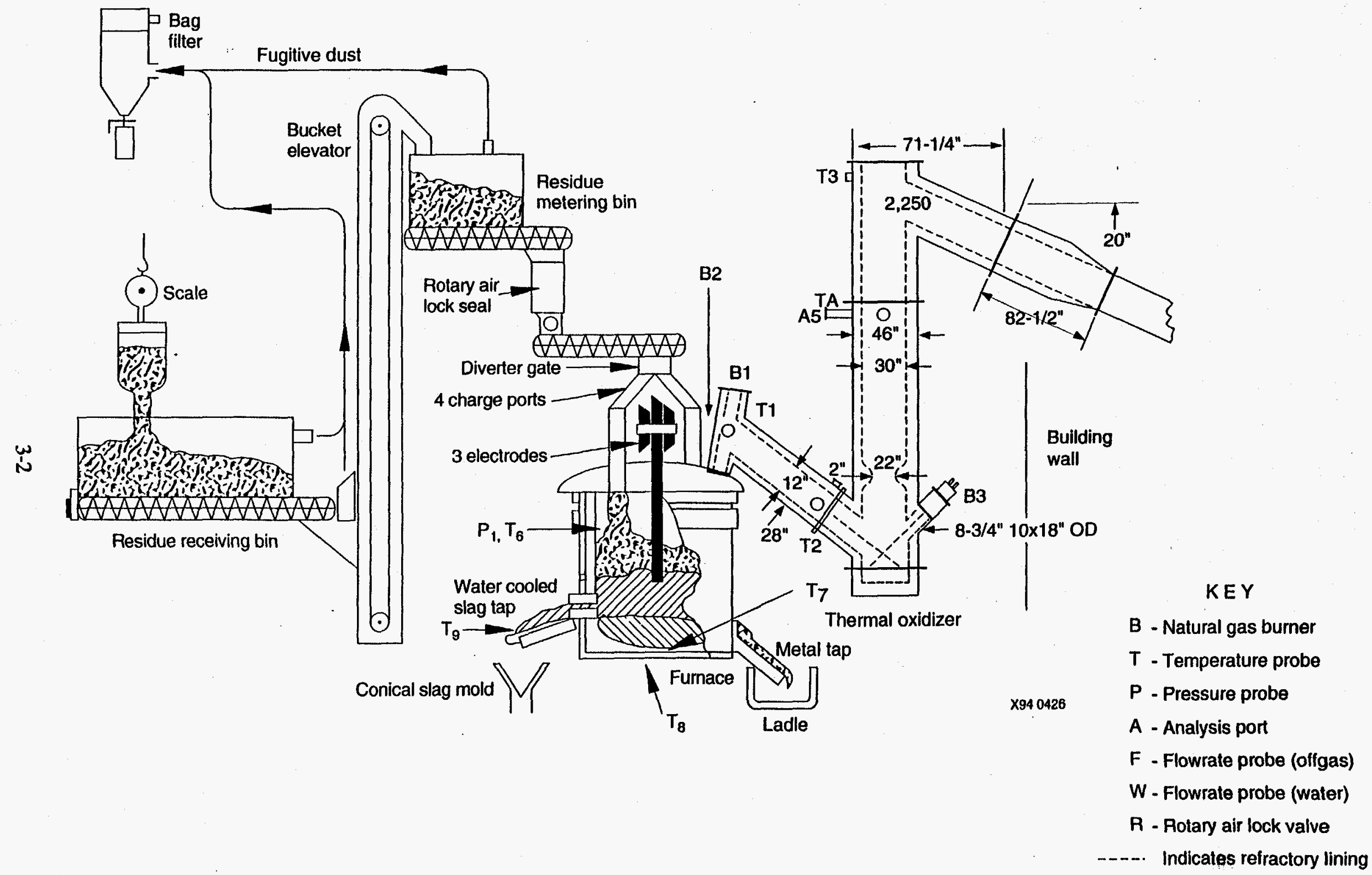

Figure 3-1. Schematic of the feed system, furnace, and thermal oxidizer. 


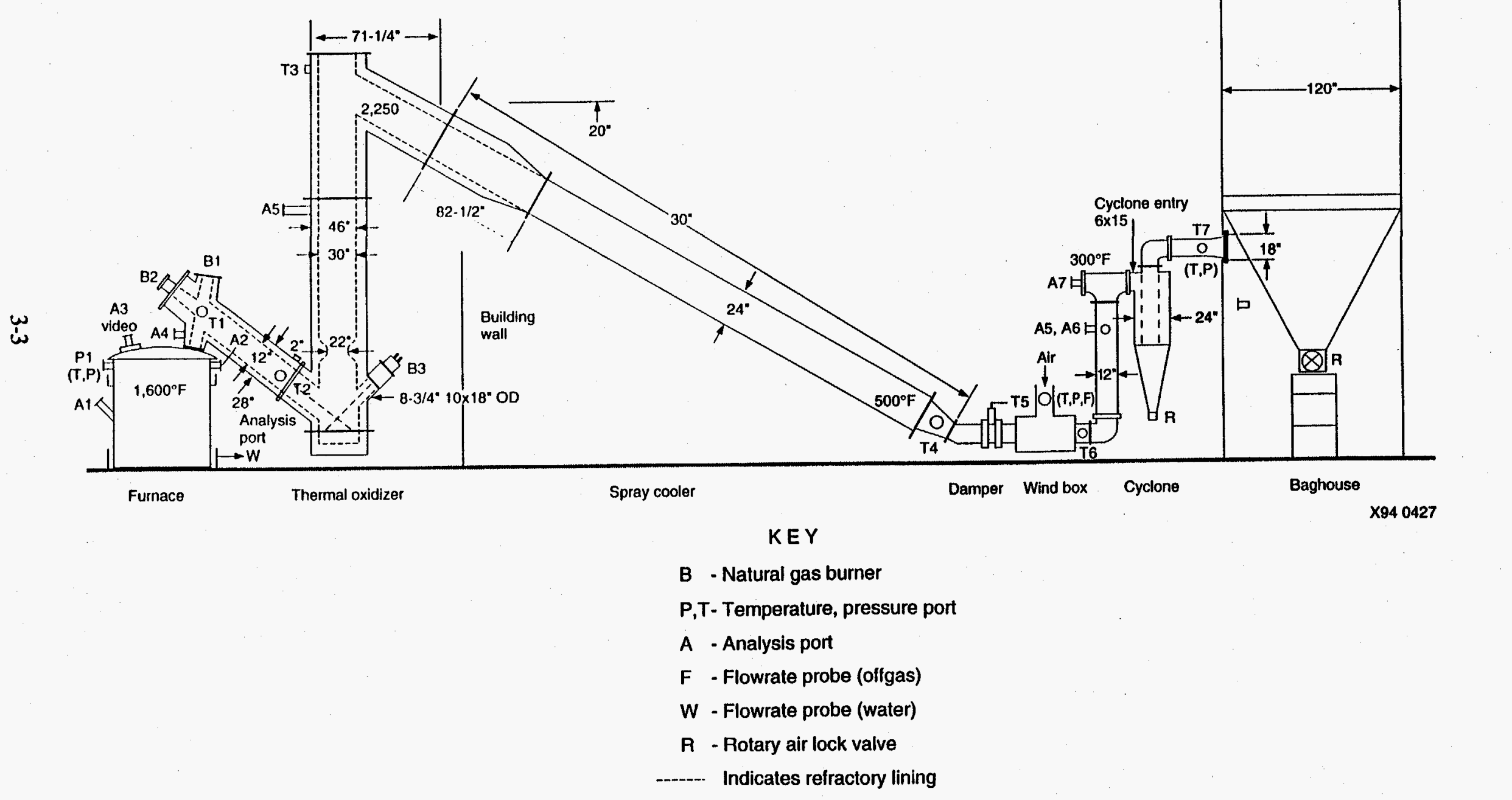

Figure 3-2. Schematic of the offgas system from the furnace to the baghouse. 




KEY

T - Temperature probe

P - Pressure probe

A - Analysis port

F - Flowrate probe (offgas)

W - Cooling water flowrate

CW - Cooling water

Figure 3-3. Schematic of the offgas system downstream of the baghouse. 


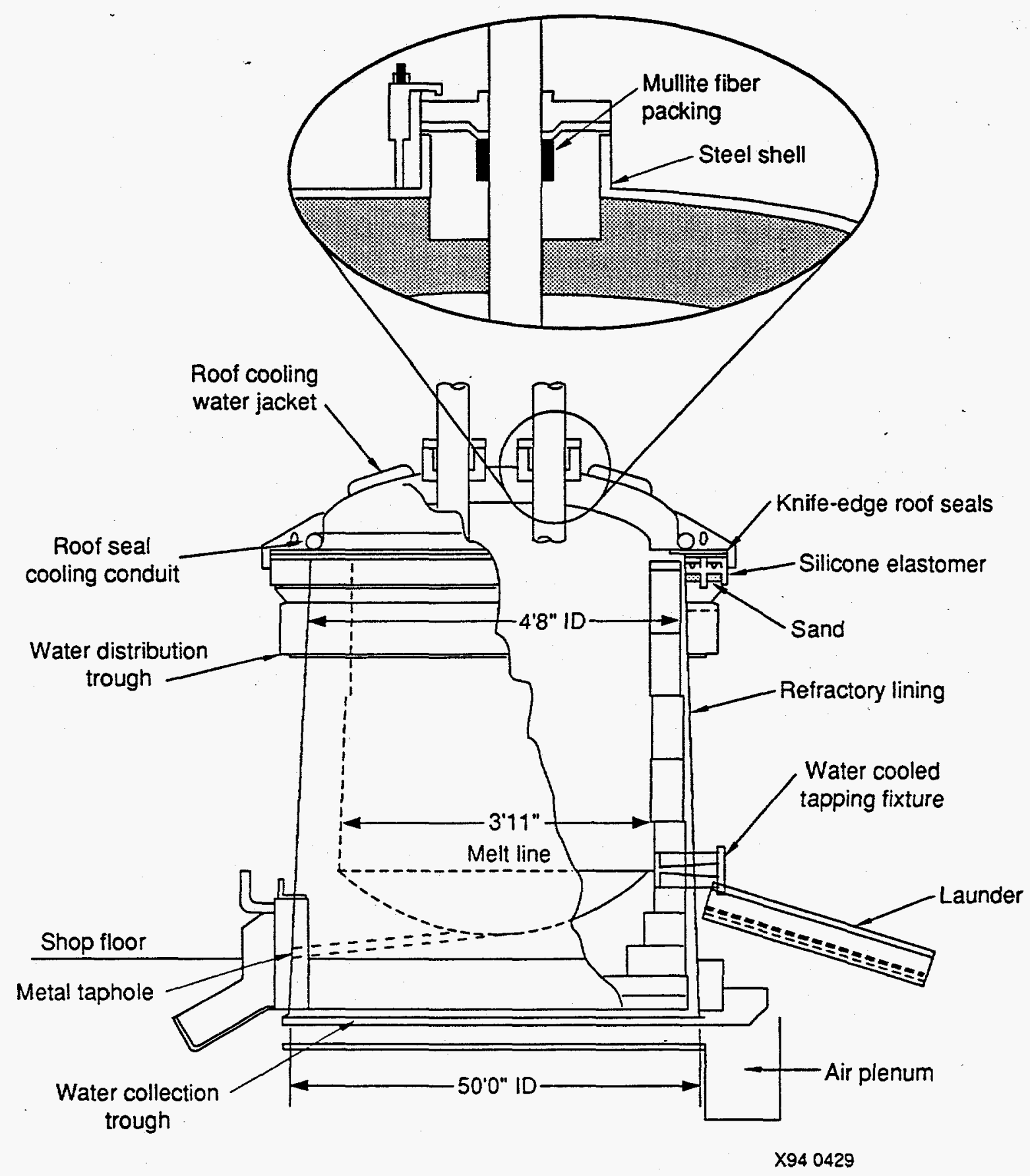

Figure 3-4. Schematic of the arc melter furnace. 
the shell. These straps were worked between the bricks and through the refractory ramming mix during construction of the hearth to provide an electrical circuit to ground between each pair of electrodes. The furnace shell was then securely grounded on the outside.

The base of the furnace is cooled by air flowing at about $1,300 \mathrm{cfm}$ through a plenum formed by six, 4-in. high "I" beams on which the furnace rests. The furnace shell, roof, slag tap hole, slag launder, metal tap hole collar, power supply transformer, and electrode arms, cables, and clamps are all water cooled. Cooling water is pumped to the furnace from a 1,500 gal, cool water sump by a $15 \mathrm{hp}$ centrifugal pump. The furnace shell is cooled by a curtain of water (50 gpm) cascading down the exterior wall from the annular distribution trough near the shell top. All cooling water is collected at the base of the furnace in a trough and is returned by gravity to a 1,500 gal, warm water sump through a 6-in. polyvinyl chloride line. Water is pumped from the warm water sump to a Marley Model NC-111 cooling tower with a cross-flow induced draft design. The cooling tower supplies up to $150 \mathrm{gpm}$ of water at $74^{\circ} \mathrm{F}$ with $142^{\circ} \mathrm{F}$ maximum return temperature and $70^{\circ} \mathrm{F}$ wet bulb ambient air temperature.

City water is available for emergency cooling in case of general power failure or pump malfunction. The most critical component is the copper, water-cooled slag tap hole (cinder monkey), which is in direct contact with molten slag while the furnace is operating. All other components should not be damaged by loss of water if furnace power is shut off. A flowmeter in the water circuit to the cinder monkey de-energizes and opens the automatic valve in the city water line if power fails or the flowrate from the cooling tower decreases below $\mathbf{2 0} \mathbf{g p m}$ in that circuit.

\subsubsection{Air Pollution Control System}

The new APCS is designed to completely oxidize the furnace offgases and control particulate, toxic metals, and acid gas emissions. The APCS will include

- A thermal oxidizer, close-coupled to the melter, designed for a maximum temperature of $2,400^{\circ} \mathrm{F}$. Two natural gas burners are located between the outlet of the furnace, and a third burner is located near the inlet of the thermal oxidizer.

- A water spray cooler for initial temperature quenching to $500^{\circ} \mathrm{F}$, followed by an airdilution temperature quench section for cooling to $300^{\circ} \mathrm{F}$.

- A cyclone for removing coarse particulate upstream of the baghouse.

- A pulse-jet baghouse for particulate removal.

- A wet scrubber for acid gas (primarily $\mathrm{HCl}$ and $\mathrm{SO}_{2}$ ) removal, with a design gas outlet temperature of around $153^{\circ} \mathrm{F}$.

- A cooler/condenser for further offgas cooling and removing condensed water droplets, with a design outlet temperature of $90^{\circ} \mathrm{F}$. 
- A resistance-heated reheater to raise the offgas above the dewpoint, with a design gas outlet temperature of $105^{\circ} \mathrm{F}$. The reheater is necessary to prevent moisture condensation in the downstream equipment and to minimize the steam plume at the stack outlet.

- A high efficiency particulate air (HEPA) filter

- An activated carbon filter for controlling $\mathrm{Hg}$ and other gaseous toxic metals and contaminants.

The baghouse for this system is the same existing baghouse used in the Phase 1 tests. The baghouse was designed for operation in the temperature range of 250 to $400^{\circ} \mathrm{F}$. There are 47 Gortex-membrane Teflon-coated fiberglass bags, 4.5 in. diameter $\times 10 \mathrm{ft}$ long. These bags are rated to remove $99.98 \%$ of particulate greater than $0.3 \mu \mathrm{m}$. Bags are cleaned with a back pulse-jet of air at 100 psig. This baghouse is rated for a maximum gas flowrate of $2,000 \mathrm{acfm}$. Additional particulate filtration is provided downstream of the baghouse by a HEPA filter unit, also rated to remove $99.98 \%$ of particulate greater than $0.3 \mu \mathrm{m}$.

\subsubsection{Phase 2 Facility Modifications}

To enable the processing of surrogate waste mixtures containing combustibles, metals, nitrates, hydroxides, and solvents, a number of modifications are being made before the shakedown and demonstration tests. These are summarized as follows:

- The length of the receiving bin will be decreased to $6 \mathrm{ft}$ to diminish classification and segregation of feed materials by the feed screws.

- A sloping deck screen will be added between the receiving bin and bucket elevator to remove objects with large aspect ratios. Screw conveyors are prone to fouling if the major diameter of particles within the feed mixture exceeds the clearance between screw and housing. The ash residues from SCE may contain objects with large aspect ratio.

- The four water-cooled feed tubes will be connected to a single-metered air supply. Combustion air will be injected through the feed tubes to provide oxidizing agent uniformly near the surface of the molten slag.

- The furnace hearth will be relined with Ruby ramming mix. Sidewall and roof refractory are in satisfactory condition.

- The APCS described in Section 3.1.2 is an extensive modification of the prior APCS used in the FY-93 tests, with an added thermal oxidizer, spray quench section, cyclone, larger baghouse, wet scrubbing, reheating, and charcoal filtration.

\subsubsection{Sample Locations and Sample Ports}

The offgas monitoring and sample collection program for the demonstration tests will be extensive to characterize furnace emissions and APCS performance. Penetrations are planned for sample ports at the locations specified in Table 3-1. Sample ports upstream of the ID fan will be 
Table 3-1. Furnace and APCS sample locations and ports.

\section{Location}

Existing port in furnace sidewall

Furnace upper sidewall above water trough

Furnace upper sidewall above water trough

On the existing door on furnace roof

In furnace outlet duct

$\underset{\infty}{\infty}$ At crossover

In wall of thermal oxidizer inlet duct

In wall of thermal oxidizer near outlet

Thermal oxidizer outlet (spray cooler inlet)

Spray cooler outlet (dilution quench inlet)

Dilution air inlet duct

Dilution quench outlet (cyclone inlet)

Dilution quench outlet (cyclone inlet)
Port no. Port specification

1.5-in. nom. pipe w/ball valve and cap

A2 1.5-in. nom. pipe w/ball valve and cap

-

A3

4-in. nom. flanged port with pipe w/ball valve and cap

A4 1.5-in. nom. pipe w/ball valve and cap

T1 -

$\mathrm{T} 2 \quad-$

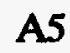

2-in. nom. pipe w/ball valve and cap

\section{T3}

T4

T5

T6

A5, A6 3-in. nom. pipe w/ball valve and cap; location per EPA specifications
Possible kinds of meastirements

Bath temperatures, gas temperatures, $\mathrm{O}_{2}, \mathrm{CO}_{2}, \mathrm{CO}$, THC, slag/metal samples

Gas temperatures, $\mathrm{O}_{2}, \mathrm{CO}_{2}, \mathrm{CO}, \mathrm{THC}$, particulate, metals, particle size

Continuous furnace pressure, temperature

Visual observations with video camera

Gas temperatures, $\mathrm{O}_{2}, \mathrm{CO}_{2}, \mathrm{CO}$, THC, particulate, metals, particle size

Continuous gas temperature - pilot burner control thermocouple tied to pilot burner (B1)

Continuous gas temperature - control thermocouple for crossover duct burner (B2)

Gas temperatures, $\mathrm{O}_{2}, \mathrm{CO}_{2}, \mathrm{CO}, \mathrm{THC}$, particulate, metals, particle size

\section{Continuous gas temperature}

Continuous gas temperature

Continuous dilution air pressure, temperature, flowrate

Continuous offgas pressure, temperature

Gas temperatures, $\mathrm{O}_{2}, \mathrm{CO}_{2}, \mathrm{CO}, \mathrm{THC}$, particulate, metals, particle size 
Table 3-1. (continued).

\begin{tabular}{|c|c|c|c|}
\hline Location & Port no. & Port specification & Possible kinds of measurements \\
\hline Flanged $\mathrm{T}$ at cyclone inlet (cyclone inlet) & A7 & $\begin{array}{l}\text { 2-in. nom. pipe } \\
\text { w/ball valve and cap }\end{array}$ & Gas composition \\
\hline Cyclone outlet (baghouse inlet) & $T 7$ & - & Continuous gas pressure, temperature \\
\hline Baghouse outlet (scrubber inlet) & $\mathrm{T} 8$ & - & Continuous gas pressure, temperature, flowrate \\
\hline Baghouse outlet (scrubber inlet) & A8 & $\begin{array}{l}\text { 2-in. nom. pipe } \\
\text { w/ball valve and cap }\end{array}$ & Gas composition \\
\hline Scrubber outlet (cooler/condenser inlet) & T9 & - & Continuous gas pressure, temperature \\
\hline Scrubber outlet (cooler/condenser inlet) & A9 & $\begin{array}{l}\text { 2-in. nom. pipe } \\
\text { w/ball valve and cap }\end{array}$ & Gas composition \\
\hline Cooler/condenser outlet (reheater inlet) & $\mathrm{T} 10$ & - & Continuous gas pressure, temperature \\
\hline Reheater outlet (ID fan inlet) & $\mathrm{T} 11$ & - & Continuous gas pressure, temperature \\
\hline HEPA filter inlet & $\mathrm{T} 12$ & - & Continuous gas pressure, temperature \\
\hline Stack & T13 & - & Continuous gas pressure, temperature, flowrate \\
\hline Stack & $\begin{array}{l}\text { A10, } \\
\text { A11 }\end{array}$ & $\begin{array}{l}\text { 3-in. nom. pipe } \\
\text { w/cap; location per } \\
\text { EPA specifications }\end{array}$ & Particulate, metals \\
\hline Stack & A12 & $\begin{array}{l}\text { 2-in. nom. pipe } \\
\text { w/ball valve and cap }\end{array}$ & Gas composition \\
\hline
\end{tabular}


designed to minimize air inleakage due at the design negative pressure of around 0.5 in. in the furnace up to around $10 \mathrm{in}$. at the inlet of the $\mathrm{W}$ fan. Minimizing air inleakage will reduce the amount of pressure and flowrate excursions in the furnace and APCS and improve worker safety.

\subsection{Test Program}

During the field test, instrumentation will be monitored and samples will be collected to characterize the performance of the melter and offgas systems. The test matrix is shown in Table 3-2. Table 3-3 shows the types of measurements and samples that will be collected for each of the test conditions. Operating parameters that will be varied during the test program are

- $\quad$ Feed mixture [RDF ash with and without an asbestos surrogate and BWID nominal waste surrogate mixture with varying (decreasing) amounts of added soil]

- Cold top (with and without)

- Oxidant addition [solid (nitrates and mill scale) in feed, and air lanced in through the feed tubes or lance-off/on and also rate]

- Power density.

These parameters are related to the ability of the furnace to process real wastes that contain various metals that may be volatile and organics that include both volatile organic and fixed carbon components. Varying the cold top and power density can affect the volatilization of metals, while oxidant addition will affect the oxidation of the fixed carbon. Volatile organics will evolve with or without oxidant addition.

The ability to form a ceramic waste will also be evaluated by using 2 TC-instrumented drums for slow-cooling the slag while monitoring the temperatures and cooling rates at different locations in the drums. Analyses of these drums will be used to evaluate the formation of crystalline structures at different cooling rates.

Operating parameters that will not be purposely varied (but they may vary during the tests) and will be monitored include

- Afterburner temperature

- Baghouse $\mathrm{dP}$, cleaning cycle, temperature, and cloth/gas ratio

- Scrubber parameters [recycle rate, make-up rate, caustic/ $\left(\mathrm{SO}_{2}+\mathrm{HCl}\right)$ ratio, gas/liquid ratio]

- Carbon absorber parameters (gas flowrate/surface area ratio, hours operated on same charcoal, and temperature)

- Redox conditions in the slag and plenum (excess $\mathrm{O}_{2}$ in the plenum just above the melt; slag and metal $\mathrm{C}$ content and $\mathrm{Fe}^{+2} / \mathrm{Fe}^{+3}$ ratio) 
Table 3-2. Test matrix for the demonstration tests.

\begin{tabular}{|c|c|c|c|c|c|c|c|}
\hline Day & $\begin{array}{l}\text { Test } \\
\text { duration } \\
\text { (hours) }\end{array}$ & $\begin{array}{l}\text { Test } \\
\text { start/stop }\end{array}$ & $\begin{array}{l}\text { Test } \\
\text { no. }\end{array}$ & Test objective & Feed mixture & $\begin{array}{l}\text { Cold } \\
\text { top }\end{array}$ & $\begin{array}{l}\text { Oxidant } \\
\text { addition }\end{array}$ \\
\hline 1 & 10 & $\begin{array}{l}1000 / 1800 \\
\text { tap metal } \\
\text { hole }\end{array}$ & 1 & $\begin{array}{l}\text { RDF bottom ash } \\
\text { demonstration }\end{array}$ & $\begin{array}{l}\text { RDF-0, bottom ash } \\
\text { with fluidizer }\end{array}$ & $\mathbf{Y}$ & $\mathbf{N}$ \\
\hline 1 & 10 & $\begin{array}{l}2000 / 0400 \\
\text { tap metal } \\
\text { hole }\end{array}$ & 1 & $\begin{array}{l}\text { RDF bottom ash } \\
\text { w/asbestos surrogate }\end{array}$ & $\begin{array}{l}\text { RDF-10A, bottom ash } \\
\text { spiked with serpentine }\end{array}$ & $\mathbf{Y}$ & $\mathbf{N}$ \\
\hline 2 & 6 & $\begin{array}{l}0600 / 1000 \\
\text { end melt }\end{array}$ & 2 & $\begin{array}{l}\text { Stepwise increase of } \\
\text { nominal waste } \\
\text { mixture in soil }\end{array}$ & $\begin{array}{l}\text { Nom-90, } 90 \% \text { soil, } \\
10 \% \text { nominal waste } \\
\text { surrogate }\end{array}$ & $\mathbf{Y}$ & $\begin{array}{l}\text { Stoic air } \\
\text { through feed } \\
\text { tubes for CO }\end{array}$ \\
\hline 2 & 6 & $\begin{array}{l}1200 / 1600 \\
\text { end melt }\end{array}$ & 2 & $\begin{array}{l}\text { Stepwise increase of } \\
\text { nominal waste } \\
\text { mixture in soil }\end{array}$ & $\begin{array}{l}\text { Nom- } 80-1,80 \% \text { soil, } \\
20 \% \text { nominal waste } \\
\text { surrogate }\end{array}$ & $\mathbf{Y}$ & $\begin{array}{l}\text { Stoic air } \\
\text { through feed } \\
\text { tubes for CO }\end{array}$ \\
\hline 2 & 6 & $\begin{array}{l}1800 / 2200 \\
\text { tap metal } \\
\text { hole }\end{array}$ & 2 & $\begin{array}{l}\text { System performance } \\
\text { evaluation }\end{array}$ & $\begin{array}{l}\text { Nom- } 80-2,80 \% \text { soil, } \\
20 \% \text { nominal waste } \\
\text { surrogate }\end{array}$ & $\mathbf{Y}$ & $\begin{array}{l}\text { Stoic air } \\
\text { through feed } \\
\text { tubes for CO }\end{array}$ \\
\hline 3 & 6 & $\begin{array}{l}0000 / 0400 \\
\text { tap metal } \\
\text { hole }\end{array}$ & 2 & $\begin{array}{l}\text { Stepwise increase of } \\
\text { nominal waste } \\
\text { mixture in soil }\end{array}$ & $\begin{array}{l}\text { Nom- } 70-1,70 \% \text { soil, } \\
30 \% \text { nominal waste } \\
\text { surrogate }\end{array}$ & $\mathbf{Y}$ & $\begin{array}{l}\text { Stoic air } \\
\text { through feed } \\
\text { tubes for CO }\end{array}$ \\
\hline 3 & 6 & $\begin{array}{l}0600 / 1000 \\
\text { end melt }\end{array}$ & 2 & $\begin{array}{l}\text { Stepwise increase of } \\
\text { nominal waste } \\
\text { mixture in soil, } \\
\text { lower power density } \\
\text { and feedrate }\end{array}$ & $\begin{array}{l}\text { Nom- } 60-1,70 \% \text { soil, } \\
30 \% \text { nominal waste } \\
\text { surrogate with added } \\
\text { mill scale }\end{array}$ & $\mathbf{Y}$ & $\begin{array}{l}\text { Stoic air } \\
\text { through feed } \\
\text { tubes for CO }\end{array}$ \\
\hline 3 & 6 & $\begin{array}{l}1200 / 1600 \\
\text { tap metal } \\
\text { hole }\end{array}$ & 3 & $\begin{array}{l}\text { Added solid oxidant } \\
\text { in feed }\end{array}$ & $\begin{array}{l}\text { Nom-60-2, } 70 \% \text { soil, } \\
30 \% \text { nominal waste } \\
\text { surrogate }\end{array}$ & $\mathbf{Y}$ & $\begin{array}{l}\text { Substoic air } \\
\text { through feed } \\
\text { tubes for CO }\end{array}$ \\
\hline 3 & 6 & $\begin{array}{l}1800 / 2200 \\
\text { tap metal } \\
\text { hole }\end{array}$ & 3 & $\begin{array}{l}\text { Added air through } \\
\text { lance for oxidizing } \\
\text { metal and fixed } \\
\text { carbon }\end{array}$ & $\begin{array}{l}\text { Nom-60-3, } 60 \% \text { soil, } \\
40 \% \text { nominal waste } \\
\text { surrogate }\end{array}$ & $\mathbf{Y}$ & $\begin{array}{l}\text { Substoic air } \\
\text { through feed } \\
\text { tubes for CO }\end{array}$ \\
\hline 4 & 6 & $\begin{array}{l}0000 / 0400 \\
\text { tap metal } \\
\text { hole }\end{array}$ & 3 & $\begin{array}{l}\text { Operate without } \\
\text { cold top }\end{array}$ & $\begin{array}{l}\text { Nom- } 60-4,60 \% \text { soil, } \\
40 \% \text { nominal waste } \\
\text { surrogate with added } \\
\text { mill scale }\end{array}$ & $\mathbf{N}$ & $\begin{array}{l}\text { Stoic air } \\
\text { through feed } \\
\text { tubes for CO }\end{array}$ \\
\hline
\end{tabular}

Note: Depending on results of the shakedown tests and from days 1-3 of the demonstration tests, the test conditions may be modified by more rapidly decreasing the proportion of soil and additives in the feed, to obtain test results at soil proportions less than $60 \%$. 
Table 3-3. Sample collection and measurement matrix for the demonstration tests.

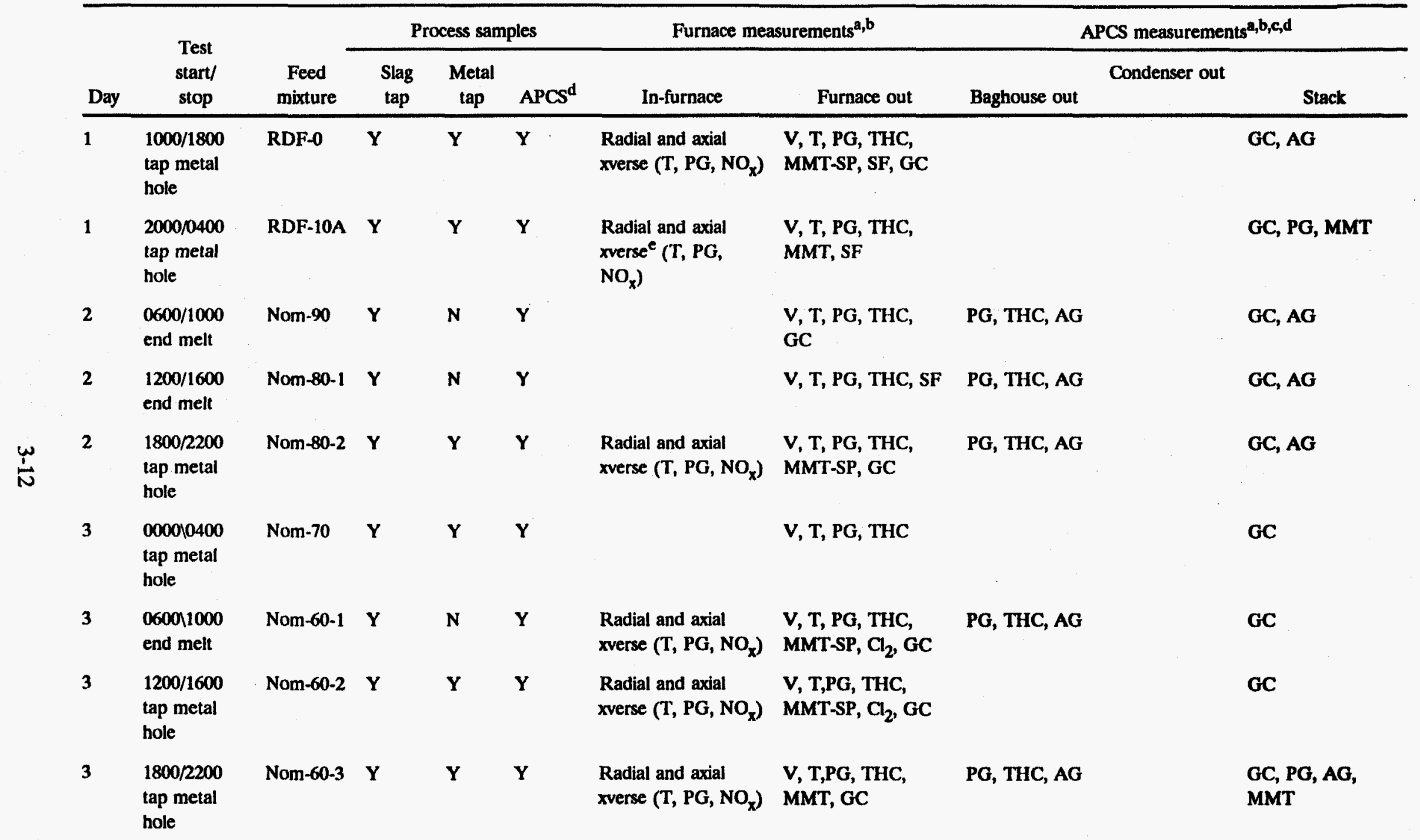


Table 3-3. (continued).

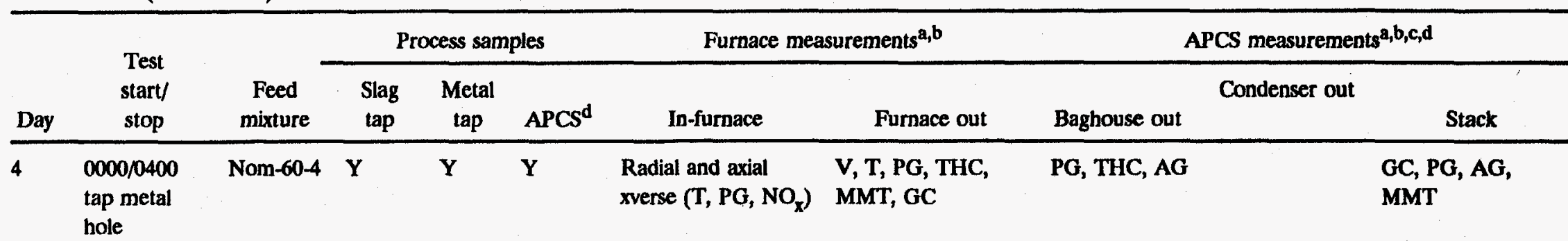

a. If possible, additional measurements such as in-situ particle sizing and FTIR gaseous analysis will also be performed at selected locations. Inclusion of additional analysis may prevent the performance of some analyses such as the $\mathrm{Cl}_{2}$ analysis. Also, the number and types of furnace and APCS measurements may change due to (a) operating information obtained during shakedown tests, (b) changes in the test matrix, or (c) limited BWID funding.

b. Measurements include V (velocity and flowrate), $\mathrm{T}$ (temperature), $\mathrm{SF}=\mathrm{Slagging} /$ fouling potential, $\mathrm{PG}$ (permanent gases $\mathrm{O}_{2}, \mathrm{CO}, \mathrm{CO}$ ), $\mathrm{AG}$ (acid gases $\mathrm{NO} \mathrm{SO}_{2}, \mathrm{SO}_{2}$ $\mathrm{HCl}$ ), THC (total hydrocarbons), $\mathrm{GC}$ (gas chromatograph analysis for $\mathrm{O}_{2}, \mathrm{CO}_{2}, \mathrm{CO}, \mathrm{Ar}, \mathrm{N}_{2}, \mathrm{H}_{2}, \mathrm{C}_{1}-\mathrm{C}_{4}, \mathrm{CCl}$, and TCE), MMT (multiple metals train for particulate and metals), MMT-SP (MMT modified for single point, abbreviated sampling to obtain grab samples of solids and vaporized materials).

$\underset{\omega}{\omega}$ c. Continuous $\mathrm{O}_{2}$ measurement is made at the outlet of the spray quench unit in addition to the above listed measurements for thermal oxidizer control.

d. APCS samples = Solid samples collected from all collection points in system (particularly from the thermal oxidizer, spray quench and baghouse).

e. xverse $=$ traverse across a diameter or radius for sample collection or measurements at discrete sample points. 
- Feedrate and power levels

- Furnace offgas flowrate, temperature, composition

- Slag temperature, viscosity, basicity

- Entrained and volatilized particulate.

\subsubsection{Feed Mixtures}

The feed mixtures will be prepared to test the operation of the melter system with bottom ash from the SCE RDF and mixtures of surrogate buried wastes and soil at the INEL RWMC. The RDF bottom ash will be tested with and without the addition of $10 \mathrm{wt} \%$ serpentine as a surrogate for asbestos. The composition of the RDF bottom ash mixtures will be determined by analysis at the ALRC.

The two mixtures of RDF bottom ash will be tested in 2 test days, 1 day for each mixture. The bottom ash will likely require pelletization before to melting for better dust control during feeding and handling. Pelletization will likely require moisture addition but may not require the addition of a binder. A drying step must follow pelletization. The decision to pelletize will be made following examination of the ash.

The surrogate buried waste mixtures will simulate the BWID nominal waste mixture mixed with soil. The target proportion of soil in the mixture is $60 \%$, but the actual maximum proportion used for the demonstration tests will be determined during the shakedown test and during the initial buried waste demonstration tests. For the demonstration tests, the actual feed mixture will be varied slightly to evaluate melter system operation with and without solid nitrate oxidants. The surrogate buried waste mixtures will also be buffered with lime $(\mathrm{CaO})$ additive to a molar basicity of 0.7 .

The composition of the buried waste materials is shown in Table A-1 in Appendix A. These waste materials include metals, solid combustibles, silicated organics, nitrate salts, and metal hydroxide sludges. The BWID nominal composition is a weighted average blend of these materials. The surrogate buried waste feed mixtures will be prepared by ALRC from representative materials blended with RWMC soil and pebble lime. The target compositions of the nominal buried waste in mixtures with 60 to $90 \%$ soil (denoted Nom-60 to Nom-90) are shown in Table A-2. The target amounts of volatile and combustible materials and elemental metals in these mixtures are summarized in Table A-3. The estimated amounts and composition of the offgases generated from melting the surrogate waste mixtures are shown in Appendix B. The estimated compositions of the surrogate additives used to prepare the surrogate waste mixtures are shown in Appendix $C$.

\subsubsection{Test Uncertainties}

The greatest uncertainties for this test program are the (a) meiter system performance characteristics while processing the surrogate INEL waste feedstreams, (b) ability to perform some of the planned diagnostics and sample collection, and (c) available funding. These uncertainties specifically include 
- Feeding the mixtures containing a variety of materials of different sizes (less than $3 / 8$ in.), shapes, and densities. Challenges include minimizing stratification, controlling dust evolution from the feed system, controlling and monitoring the feedrate, and blockage at auger locations, feed tubes, and other points.

- Performance of the melter when processing feed mixtures containing large amounts of volatile organics, fixed carbon, and nitrates. Challenges include pressure fluctuation control, volatilization of volatile materials, oxidation of fixed carbon, minimization of soot deposition in the furnace (that may interfere with arc performance), and deposition of other materials.

- Performance of the air pollution control system. Challenges include minimizing fouling deposits throughout the system, achieving efficient oxidation of organics in the thermal oxidizer, sufficient offgas cooling to protect the baghouse without excessive cooling and resultant moisture condensation, and efficient particulate and acid gas control.

- Performance of monitoring and sample collection equipment. Measurements and sampling are planned for many different locations in the melter system including the furnace and selected locations in the APCS. Due to high temperatures and high levels of entrained particulate at these locations, the success of some of the measurement and sampling methods will need to be demonstrated during the shakedown test and demonstration tests.

- Ability to perform measurements at locations between the furnace outlet and thermal oxidizer outlet. The presence of burner flames will impact the ability and interpretation of measurements, so burners will be turned off if having the burners off is considered a safe practice.

The test program and QA program have been developed to specifically address these uncertainties. As much as is reasonably possible, melter system performance and configuration of the offgas sampling and analytical equipment and procedures will be evaluated in the shakedown tests. These evaluations will include measuring temperatures, velocities, gaseous composition, and particulate levels in the furnace and at the furnace outlet, baghouse outlet, and stack. Samples of slag and metal (if produced) will be collected during tapping and, if necessary, through ports in the furnace. Samples of APCS solids will be collected at all collection points in the APCS. Following the shakedown test, the melter system will be inspected for indicators of feed plugging, fouling, erosion, corrosion, and any other visible conditions.

There may be insufficient funding to perform the full scope of test measurements and sample collection described in Section 3.2. Engineering judgement and results obtained in the shakedown tests and initial demonstration tests will be used to limit the scope of testing to available funding.

\subsubsection{Contingency Plans}

Contingency plans for potential undesired occurrences include identifying (to the extent possible) the cause of the occurrence and taking steps to prevent recurrence. Depending on the circumstance, resolution may include equipment or operational changes to correct the situation or revising the test plan to avoid the occurrence. Test plan revisions may be done by and with the approval of the onsite 
test team leaders, and documented in test log notes. Such revisions will be made only when needed to ensure safe operations and maximize the amount and quality of the test results.

Feed system problems must generally be resolved for continued testing by more direct manual interaction, equipment modifications, or changing the feed mixture. Furnace problems can vary widely but can often be remedied by adjusting the feedrate or power levels, electrode control (rheostat), or feed mixture composition. Adjustments are also available for all components of the APCS to optimize the performance of the thermal oxidizer, cooler, baghouse, scrubber, charcoal filter, and HEPA filter.

Furnace plenum and APCS sample collection and monitoring procedures may require modification for better suitability for the sample locations, available sampling time, and offgas conditions. It may be necessary to significantly modify some of the in-furnace and furnace outlet sampling and analysis procedures because of temperature and particulate conditions in the offgas and to avoid, or account for, the three gas burners in the system. Such modifications, if necessary, will be made to meet the goals of the test program within limitations of those procedures. 


\section{SEQUENCE OF ACTIVITIES}

The success of the baseline tests depends on the performance of many activities by the USBM ALRC and INEL. The demonstration tests cannot be performed until completion of several activities. These activities include

- APCS modifications: (a) designing overall system, (b) designing individual components, (c) purchasing or fabricating APCS components, (d) installation, (e) fabricating and installing sample ports, and (f) operational testing

- Feed system modifications: (a) shortening the receiving bin length, (b) fabricating and installing the sloping deck screen, (c) fabricating and installing the feed tube air injection system, and (d) designing and fabricating the air lance

- Furnace preparations: (a) refractory casting, (b) added instrumentation, (c) final assembly, and (d) operational checkout

- Feed mixture preparations: (a) shipping RDF bottom ash, (b) acquisition of buried waste additives, (c) sample analysis of RDF bottom ash, soil and feed additives, and (d) mixing the feed materials as determined prior to the demonstration tests

- Screening tests in the " $\mathrm{W}$ " furnace for evaluating refractory performance, melting temperatures, offgas emissions, and safety

- Shakedown test

- Review and revisions following shakedown test

- Demonstration tests

- Post-test activities: (a) equipment inspections, (b) data management, and (c) sample handling and analysis

- Field data report and draft and final data evaluation reports.

This document is not a controlling document for project test schedule. For information purposes, the estimated sequence of events is as follows: 
Overall APCS system design-Completed

Milestone: APCS design meeting, Denver, CO_Completed in October 1993

Design of individual APCS components-Completed

Draft of test plan-Completed

Placing on order all APCS components-Completed

Feed mixture preparations

Feed system modifications

Furnace preparations

"W" furnace screening tests

Receiving all APCS components

Installation of furnace and APCS modifications

Final test plan

Initial shakedown test

Final shakedown test

Demonstration tests

Post-test sample and data handling

Quick look field data test report

Laboratory sample analyses

Test results report 


\section{MEASUREMENTS AND SAMPLE COLLECTION}

The sampling and analysis plan is provided in this test plan rather than in the technology test plan. There is currently insufficient BWID funding for the full scope of sample collection and analysis described here. Furthermore, some of the measurements described here may be deleted based on results of the shakedown tests, while other measurements may be added. Sound engineering judgement will be used to perform the testing within the funding available at the time of the demonstration tests and meet the test objectives.

\subsection{Melter Furnace Process Continuous Monitoring}

Facility process monitoring and data acquisition will be performed using a computer data acquisition system (DAS) and by using manually recorded logs and data sheets. Printouts of the DAS-recorded data will be automatically made every 2 minutes. The monitored parameters are summarized in Table 5-1.

\subsection{Process Sample Collection}

Process material samples that will be collected during or after each test are summarized in Table 5-2. Analytical results of these samples will be used to (a) perform process material mass balances, (b) determine product phase partitioning and fate of metal and surrogate TRU elements in the feed, (c) determine if the secondary product (APCS) streams are hazardous, and (d) evaluate the physical and chemical properties of the vitrified slag and metal phases. Process sample collection and handling procedures are summarized in Table 5-3.

\subsection{Gaseous and Particulate Measurements and Sample Collection}

Gaseous and particulate measurements and sample collection, in addition to the process monitoring and sample collection described in Sections 5.1 and 5.2, will be conducted at selected locations in the furnace and the APCS. Gaseous measurements will include velocity, temperature, and composition measurements. Particulate measurements will include slagging/fouling potential, total particulate, and metals. The total particulate and metals determinations will be done using the draft EPA Method 29, or MMT, modified to include measurement of total particulate in addition to metals. All measurements in the high-temperature areas upstream of the spray cooler will be done using water or air-cooled sample probes. Gaseous and particulate measurement and sample collection procedures are summarized for the different sample locations in Table 5-4. Sampling periods of up to several hours for each test condition may be required to complete the planned scope of sample collection and offgas measurements.

Sample collection and measurements using probes inserted through sample ports upstream of the ID fan can affect the-furnace and APCS operation by allowing ambient air leakage into the system if the sample ports are not well sealed around the sample probes. Negative pressures (draft) of around 1-in. or more are necessary in the furnace to prevent hot, noxious plenum gases into the work area. The draft will increase downstream in the APCS and will probably exceed $10 \mathrm{in}$. at the inlet of the ID fan. Sealing around sampling probes in the areas of the baghouse, the scrubber and cooler/condenser will be especially important due to the high draft. Sample probes will be 
Table 5-1. ALRC melter process monitoring summary.

\begin{tabular}{|c|c|c|c|c|}
\hline \multirow[b]{2}{*}{ Parameter } & \multirow[b]{2}{*}{ Units } & \multicolumn{2}{|c|}{ Recording method } & \multirow[b]{2}{*}{ Frequency } \\
\hline & & $\begin{array}{l}\text { Continuous } \\
\text { data logger } \\
\text { (automatic) }\end{array}$ & Manual & \\
\hline \multicolumn{5}{|l|}{ Melter } \\
\hline Electrode phase to phase voltage & $\mathbf{V}$ & & $\mathbf{x}$ & once/hr \\
\hline Electrode phase to neutral voltage & $\mathbf{V}$ & $\mathbf{x}$ & & C \\
\hline Electrode current & $\mathbf{A}$ & $\mathbf{x}$ & & $\mathrm{C}$ \\
\hline Power factor & - & $\mathbf{x}$ & & $\mathrm{C}$ \\
\hline Active power & $\mathbf{k W}$ & $\mathbf{x}$ & & C \\
\hline Conductance factor & $\mathrm{I}^{2} / \mathrm{kW}$ & & $\mathbf{x}$ & once/hr \\
\hline Material feedrate & $\mathbf{l b} / \mathbf{h}$ & $\mathbf{x}$ & $\mathbf{x}$ & once/15 min. \\
\hline Total material feed & lb/test & & $\mathbf{x}$ & once/test \\
\hline Energy efficiency & $\mathbf{k W h} / \mathbf{l b}$ & & $\mathbf{x}$ & once/hr \\
\hline Cold top depth & in. & & $\mathbf{x}$ & $\mathrm{C}$, once $/ 10 \mathrm{~min}$. \\
\hline Slag tap temperature, $T_{9}$ & ${ }^{\circ} \mathrm{C}$ & $\mathbf{x}$ & & $\mathbf{C}$ \\
\hline Slag tap rate & $\mathrm{lb} / \mathrm{h}$ & & $\mathbf{x}$ & Every slag pot \\
\hline Electrode stroke position & in. & & $\mathbf{x}$ & once/15 min. \\
\hline Bath temperature & ${ }^{\circ} \mathrm{C}$ & & $\mathbf{x}$ & once/test \\
\hline Slag viscosity (estimate) & -- & & $\mathbf{x}$ & $\begin{array}{l}30 \text { minutes or } \\
\text { during taps }\end{array}$ \\
\hline Metal tap temperature & ${ }^{\circ} \mathrm{C}$ & & $\mathbf{x}$ & During taps \\
\hline Furnace static pressure & in. $\mathrm{H}_{2} \mathrm{O}$ & $\mathbf{x}$ & $\mathbf{x}$ & C \\
\hline Furnace hearth temperature, $T_{7}$ & ${ }^{\circ} \mathrm{C}$ & $\mathbf{x}$ & & C \\
\hline Furnace upper inside wall $\mathbf{T}$ & ${ }^{\circ} \mathrm{C}$ & $\mathbf{x}$ & & $\mathrm{C}$ \\
\hline Furnace plenum temperature & ${ }^{\circ} \mathrm{C}$ & $\mathbf{x}$ & & $\mathrm{C}$ \\
\hline Furnace roof temperature & ${ }^{\circ} \mathrm{C}$ & $\mathbf{x}$ & & $\mathrm{C}$ \\
\hline Hearth shell temperature, $T_{8}$ & ${ }^{\circ} \mathrm{C}$ & $\mathbf{x}$ & & $\mathrm{C}$ \\
\hline Electrode consumption & $\mathrm{lb} /$ test & & $\mathbf{x}$ & $1 /$ test \\
\hline Oxidizing air flowrate & scfm & $\mathbf{x}$ & & $\mathrm{C}$ \\
\hline Oxidizing air temperature & ${ }^{\circ} \mathrm{C}$ & $\mathbf{x}$ & & $\mathrm{C}$ \\
\hline
\end{tabular}


Table 5-1. (continued).

\begin{tabular}{|c|c|c|c|c|}
\hline \multirow[b]{2}{*}{ Parameter } & \multirow[b]{2}{*}{ Units } & \multicolumn{2}{|c|}{ Recording method } & \multirow[b]{2}{*}{ Frequency } \\
\hline & & $\begin{array}{l}\text { Continuous } \\
\text { data logger } \\
\text { (automatic) }\end{array}$ & Manual & \\
\hline \multicolumn{5}{|l|}{ Air Pollution Control System } \\
\hline Crossover gas temperature & ${ }^{\circ} \mathrm{C}$ & $\mathbf{x}$ & & $\mathbf{C}$ \\
\hline Thermal oxidizer (TO) inlet $T$ & ${ }^{\circ} \mathrm{C}$ & & & \\
\hline TO outlet temperature & ${ }^{\circ} \mathrm{C}$ & $\mathbf{x}$ & $\mathbf{x}$ & C \\
\hline TO combustion air flowrate & scfm & $\mathbf{x}$ & $\mathbf{x}$ & $\mathrm{C}$ \\
\hline TO combustion air temperature & ${ }^{\circ} \mathrm{C}$ & $\mathbf{x}$ & & $\mathrm{C}$ \\
\hline TO fuel flowrate & scfm & & $\mathbf{x}$ & 30 minutes \\
\hline Spray dryer water flowrate & gpm & $\mathbf{x}$ & $\mathbf{x}$ & $\mathrm{C}$ \\
\hline Spray dryer water temperature & ${ }^{\circ} \mathrm{C}$ & $\mathbf{x}$ & & $\mathrm{C}$ \\
\hline Spray dryer outlet temperature & ${ }^{\circ} \mathrm{C}$ & $\mathbf{x}$ & & $\mathrm{C}$ \\
\hline Quench air temperature & ${ }^{\circ} \mathrm{C}$ & $\mathbf{x}$ & & C \\
\hline Quench air pressure & ${ }^{\circ} \mathrm{C}$ & $\mathbf{x}$ & & $\mathrm{C}$ \\
\hline Quench air flowrate & $\operatorname{scfm}$ & $\mathbf{x}$ & & $\mathrm{C}$ \\
\hline Quench outlet temperature & ${ }^{\circ} \mathrm{C}$ & $\mathbf{x}$ & & $\mathrm{C}$ \\
\hline Quench outlet pressure & ${ }^{\circ} \mathrm{C}$ & $\mathbf{x}$ & & $\mathrm{C}$ \\
\hline Baghouse inlet temperature & ${ }^{\circ} \mathrm{C}$ & $\mathbf{x}$ & & $\mathrm{C}$ \\
\hline Baghouse inlet pressure & in. $\mathrm{H}_{2} \mathrm{O}$ & $x$ & & C \\
\hline Baghouse outlet temperature & ${ }^{\circ} \mathrm{C}$ & $\mathbf{x}$ & & $\mathrm{C}$ \\
\hline Baghouse outlet pressure & in. $\mathrm{H}_{2} \mathrm{O}$ & $\mathbf{x}$ & & $\mathrm{C}$ \\
\hline Baghouse outlet flowrate & $\operatorname{scfm}$ & $\mathbf{x}$ & & C \\
\hline Scrubber liquor flowrate & gpm & & $\mathbf{x}$ & once $/ 30 \mathrm{~min}$. \\
\hline Scrubber liquor temperature & ${ }^{\circ} \mathrm{C}$ & $x$ & & $\mathrm{C}$ \\
\hline Scrubber blowdown flowrate & gpm & & $\mathbf{x}$ & once $/ 30 \mathrm{~min}$. \\
\hline Scrubber blowdown temperature & ${ }^{\circ} \mathrm{C}$ & $\mathbf{x}$ & & $\mathrm{C}$ \\
\hline Scrubber liquor $\mathrm{pH}$ & - & & $\mathbf{x}$ & once $/ 30 \mathrm{~min}$. \\
\hline Scrubber outlet gas temperature & ${ }^{\circ} \mathrm{C}$ & $\mathbf{x}$ & & C \\
\hline Scrubber outlet gas pressure & in. $\mathrm{H}_{2} \mathrm{O}$ & $\mathbf{x}$ & & $\mathrm{C}$ \\
\hline Condenser water flowrate & ${ }^{\circ} \mathrm{C}$ & & $\mathbf{x}$ & once $/ 30 \mathrm{~min}$. \\
\hline
\end{tabular}


Table 5-1. (continued).

\begin{tabular}{|c|c|c|c|c|}
\hline \multirow[b]{2}{*}{ Parameter } & \multirow[b]{2}{*}{ Units } & \multicolumn{2}{|c|}{ Recording method } & \multirow[b]{2}{*}{ Frequency ${ }^{3}$} \\
\hline & & $\begin{array}{l}\text { Continuous } \\
\text { data logger } \\
\text { (automatic) }\end{array}$ & Manual & \\
\hline Condenser outlet gas temperature & ${ }^{\circ} \mathrm{C}$ & $\mathbf{x}$ & & $\mathrm{C}$ \\
\hline Condenser outlet gas pressure & in. $\mathrm{H}_{2} \mathrm{O}$ & $\mathbf{x}$ & & $\mathrm{C}$ \\
\hline Reheater power & $\mathbf{k W}$ & & $\mathbf{x}$ & once $/ 30 \mathrm{~min}$. \\
\hline Reheater outlet gas temperature & scfm & & $\mathbf{x}$ & 30 minutes \\
\hline HEPA filter inlet gas temperature & ${ }^{\circ} \mathrm{C}$ & $\mathbf{x}$ & & $\mathrm{C}$ \\
\hline HEPA filter inlet gas pressure & ${ }^{\circ} \mathrm{C}$ & $\mathbf{x}$ & & $\mathrm{C}$ \\
\hline $\begin{array}{l}\text { HEPA/carbon filter differential } \\
\text { pressure } \mathbf{P}\end{array}$ & in. $\mathrm{H}_{2} \mathrm{O}$ & $\mathbf{x}$ & & $\mathrm{C}$ \\
\hline Stack gas temperature & ${ }^{\circ} \mathrm{C}$ & $\mathbf{x}$ & & $\mathrm{C}$ \\
\hline Stack gas flowrate & scfm & $\mathbf{x}$ & & $\mathrm{C}$ \\
\hline TO catch mass & $\mathrm{lb} /$ test & & $\mathbf{x}$ & once/test \\
\hline Cyclone catch mass & lb/test & & $\mathbf{x}$ & once/test \\
\hline Baghouse hopper catch mass & lb/test & & $\mathbf{x}$ & once/test \\
\hline \multicolumn{5}{|l|}{$\begin{array}{l}\text { Cooling Water Flowrates and Outlet } \\
\text { Temperatures }\end{array}$} \\
\hline Inlet water flow & $\mathrm{gpm},{ }^{\circ} \mathrm{C}$ & & $\mathbf{x}$ & once $/ 30 \mathrm{~min}$. \\
\hline Cables, clamps, arms & $\mathrm{gpm}^{\circ} \mathrm{C}$ & & $\mathbf{x}$ & once $/ 30 \mathrm{~min}$. \\
\hline Feed tubes & $\mathrm{gpm},{ }^{\circ} \mathrm{C}$ & & $\mathbf{x}$ & once $/ 30 \mathrm{~min}$. \\
\hline Furnace roof & $\mathrm{gpm},{ }^{\circ} \mathrm{C}$ & & $\mathbf{x}$ & once $/ 30 \mathrm{~min}$. \\
\hline Furnace shell & gpm, ${ }^{\circ} \mathrm{C}$ & & $\mathbf{x}$ & once $/ 30 \mathrm{~min}$. \\
\hline Cinder monkey & $\mathrm{gpm}^{\circ} \mathrm{C}$ & & $\mathbf{x}$ & once $/ 30 \mathrm{~min}$. \\
\hline Exit duct & gpm, ${ }^{\circ} \mathrm{C}$ & & $\mathbf{x}$ & once $30 \mathrm{~min}$. \\
\hline
\end{tabular}

a. $\mathrm{C}=$ continuous, with 2 -minute automatic printout 
Table 5-2. Sample collection summary.

\begin{tabular}{|c|c|c|c|c|c|c|}
\hline \multirow[b]{2}{*}{$\begin{array}{c}\text { Feed } \\
\text { mixture }\end{array}$} & \multirow[b]{2}{*}{ Test objective } & \multicolumn{3}{|c|}{ Process samples collected ${ }^{2}$} & \multicolumn{2}{|c|}{ APCS samples collected ${ }^{\mathrm{a}, \mathrm{b}}$} \\
\hline & & $\begin{array}{l}\text { Slag } \\
\text { tap }\end{array}$ & $\begin{array}{c}\text { Metal } \\
\text { tap }\end{array}$ & $\begin{array}{l}\text { APCS (3 } \\
\text { locations) }\end{array}$ & $\begin{array}{l}\text { MMT front } \\
\text { half }\end{array}$ & $\begin{array}{l}\text { MMT bact } \\
\text { half }\end{array}$ \\
\hline RDF-0 & $\begin{array}{l}\text { RDF bottom ash } \\
\text { demonstration }\end{array}$ & 7 & 2 & 3 & 1 & 1 \\
\hline RDF-10A & $\begin{array}{l}\text { RDF bottom ash } \\
\text { w/asbestos surrogate }\end{array}$ & 7 & 2 & 3 & 2 & 2 \\
\hline Nom-90 & $\begin{array}{l}\text { Stepwise increase of } \\
\text { nominal waste } \\
\text { mixture in soil }\end{array}$ & 4 & & 3 & & \\
\hline Nom-80-1 & $\begin{array}{l}\text { Stepwise increase of } \\
\text { nominal waste } \\
\text { mixture in soil }\end{array}$ & 4 & & 3 & & \\
\hline Nom-80-2 & $\begin{array}{l}\text { System performance } \\
\text { evaluation }\end{array}$ & 4 & 2 & 3 & 1 & 1 \\
\hline Nom-70 & $\begin{array}{l}\text { Stepwise increase of } \\
\text { nominal waste } \\
\text { mixture in soil }\end{array}$ & 4 & 2 & 3 & & \\
\hline Nom-60-1 & $\begin{array}{l}\text { Stepwise increase of } \\
\text { nominal waste } \\
\text { mixture in soil, lower } \\
\text { power density and } \\
\text { feedrate }\end{array}$ & 4 & & 3 & 1 & 1 \\
\hline Nom-60-2 & $\begin{array}{l}\text { Add solid oxidant in } \\
\text { feed }\end{array}$ & 4 & 2 & 3 & 1 & 1 \\
\hline Nom-60-3 & $\begin{array}{l}\text { Added air thru lance } \\
\text { for oxidizing metal }\end{array}$ & 4 & & 3 & 2 & 2 \\
\hline Nom-60-4 & $\begin{array}{l}\text { Operate without } \\
\text { cold top }\end{array}$ & 4 & 2 & 3 & 2 & 2 \\
\hline Total samples & & 46 & 12 & 30 & 10 & 10 \\
\hline $\begin{array}{l}\text { a. The actual } \\
\text { test. }\end{array}$ & nber of samples may v & ased o & actual & rating time & nount of mater & cessed in each \\
\hline
\end{tabular}


Table 5-3. Process sample handling summary.

\begin{tabular}{|c|c|c|c|c|}
\hline $\begin{array}{l}\text { Process } \\
\text { sample }\end{array}$ & $\begin{array}{l}\text { Sample } \\
\text { location }\end{array}$ & $\begin{array}{l}\text { Sample } \\
\text { size }\end{array}$ & $\begin{array}{l}\text { Sample } \\
\text { frequency }\end{array}$ & $\begin{array}{l}\text { Sample } \\
\text { handling }\end{array}$ \\
\hline $\begin{array}{l}\text { Slag } \\
\text { product }\end{array}$ & Slag tap & $\begin{array}{l}\text { Approx. } \\
5 \mathrm{~kg} \\
\text { ladle } \\
\text { sample }\end{array}$ & $\begin{array}{l}\text { Mid-way through each pot, } \\
\text { or when considered } \\
\text { necessary via metal dip rod } \\
\text { when not tapping }\end{array}$ & $\begin{array}{l}\text { Crush each individual sample; split } \\
\text { remaining sample by coning/quartering } \\
\text { as needed to obtain analysis aliquots; } \\
\text { archive remainder }\end{array}$ \\
\hline $\begin{array}{l}\text { Metal } \\
\text { product }\end{array}$ & $\begin{array}{l}\text { Metal tap (or } \\
\text { from dip rod } \\
\text { if not } \\
\text { tapping) }\end{array}$ & $\begin{array}{l}\text { Approx. } \\
5 \mathrm{~kg} \\
\text { ladle } \\
\text { sample }\end{array}$ & $\begin{array}{l}\text { Mid-way through each pot, } \\
\text { or when considered } \\
\text { necessary via metal dip rod } \\
\text { when not tapping }\end{array}$ & Not available \\
\hline $\begin{array}{l}\text { Thermal } \\
\text { oxidizer } \\
\text { catch }\end{array}$ & $\begin{array}{l}\text { Thermal } \\
\text { oxidizer } \\
\text { hopper }\end{array}$ & $\begin{array}{l}\text { Entire } \\
\text { amount }^{a}\end{array}$ & End of each test condition & $\begin{array}{l}\text { Crush each individual sample as } \\
\text { needed; split sample by } \\
\text { coning/quartering as needed to obtain } \\
\text { analysis aliquots; archive remainder }\end{array}$ \\
\hline $\begin{array}{l}\text { Cyclone } \\
\text { catch }\end{array}$ & Cyclone & $\begin{array}{l}\text { Entire } \\
\text { amount }^{\mathrm{a}}\end{array}$ & End of each test condition & $\begin{array}{l}\text { Crush each individual sample as } \\
\text { needed; split sample by } \\
\text { coning/quartering as needed to obtain } \\
\text { analysis aliquots; archive remainder }\end{array}$ \\
\hline $\begin{array}{l}\text { Baghouse } \\
\text { catch }\end{array}$ & $\begin{array}{l}\text { Baghouse } \\
\text { hopper }\end{array}$ & $\begin{array}{l}\text { Entire } \\
\text { amount }^{\mathrm{a}}\end{array}$ & End of each test condition & $\begin{array}{l}\text { Crush each individual sample as } \\
\text { needed; split sample by } \\
\text { coning/quartering as needed to obtain } \\
\text { analysis aliquots; archive remainder }\end{array}$ \\
\hline $\begin{array}{l}\text { Scrubber } \\
\text { feedwater }\end{array}$ & $\begin{array}{l}\text { House water } \\
\text { supply }\end{array}$ & $1 \mathrm{~L}$ & Once during test program & Archive \\
\hline $\begin{array}{l}\text { Scrubber } \\
\text { caustic }\end{array}$ & $\begin{array}{l}\text { House water } \\
\text { supply }\end{array}$ & $1 \mathrm{~L}$ & Once during test program & Archive \\
\hline $\begin{array}{l}\text { Scrubber } \\
\text { blowdown }\end{array}$ & $\begin{array}{l}\text { Scrubber } \\
\text { blowdown }\end{array}$ & $1 \mathrm{~L}$ & $\begin{array}{l}\text { Once midway through each } \\
\text { test condition }\end{array}$ & $\begin{array}{l}\text { Analyze aliquots as needed; archive } \\
\text { remainder }\end{array}$ \\
\hline
\end{tabular}

a. The offgas system residues will be collected as completely as possible from the hoppers and traps in the system. 
Table 5-4. Gaseous and particulate measurement and sampling procedures.

\begin{tabular}{|c|c|c|c|c|c|c|}
\hline $\begin{array}{l}\text { Sample location (port } \\
\text { location) }\end{array}$ & Procedure & Reference & $\begin{array}{c}\text { Sampling/measurement } \\
\text { time }\end{array}$ & Sampling point matrix & Equipment & Comments \\
\hline \multirow[t]{7}{*}{$\begin{array}{l}\text { Sample ports } \\
\text { upstream of } \\
\text { baghouse }\end{array}$} & $\begin{array}{l}\text { Gas temperature by } \\
\text { suction pyrometer }\end{array}$ & EPRI CS.5552 & 20-60 minutes/traverse & $\begin{array}{l}\text { Minimum 3-point traverse } \\
\text { (far wall, near wall, } \\
\text { centerline) and single- } \\
\text { point }\end{array}$ & $\begin{array}{l}\text { Combined and separate } \\
\text { suction pyrometer and } \\
\text { S-type pitot, and gas } \\
\text { sampling probes in air } \\
\text { or water-cooled sheaths. }\end{array}$ & $\begin{array}{l}\text { Low gas velocities of around } 1-10 \mathrm{fts} \text { in } \\
\text { furnace require sensitive manometer or } \\
\text { pressure transducer, calibrated with pitot } \\
\text { at low velocities. The gas sampling } \\
\text { probes may require inertial phase } \\
\text { separation to prevent plugging. } \\
\text { Traversing and long-term sampling in } \\
\text { furnace may be limited to quartz or } \\
\text { alumina probes for safety. }\end{array}$ \\
\hline & Gas velocity & EPA Method 2 & Same as above & Same as above & Same as above & Same as above \\
\hline & $\begin{array}{l}\text { Offgas composition } \\
\text { by specific EPA } \\
\text { procedure }\end{array}$ & $\begin{array}{l}\text { EPA 3A, 6C, 7E, } \\
10,25 A\end{array}$ & Same as above & Same as above & Same as above & $\begin{array}{l}\text { Same as above with the added HCL air- } \\
\text { dilution probe }\end{array}$ \\
\hline & $\begin{array}{l}\text { Offgas composition } \\
\text { by gas chromatograph }\end{array}$ & $\begin{array}{l}\text { MTI operating } \\
\text { manual }\end{array}$ & up to $1 \mathrm{hr}$ & Same as above & Same & $\begin{array}{l}\text { Same as above, with additional gas } \\
\text { scrubbing to remove acid gases, especially } \\
\text { HCl. Samples will be collecied into } \\
\text { Tedlar bags for discrete injection and } \\
\text { protection of GC }\end{array}$ \\
\hline & Gaseous $\mathrm{Cl}_{2}$ & $\begin{array}{l}\text { EPA } 26 \text { modified to } \\
\text { include } \mathrm{Cl}_{2} \text { analysis }\end{array}$ & & & & \\
\hline & $\begin{array}{l}\text { Particle size, } \\
\text { particulate, and } \\
\text { metals }\end{array}$ & $\begin{array}{l}\text { EPA } 201 A(40 \text { CFR } \\
52, \text { App. } M) \mathrm{PM}_{10} \\
\text { procedure }\end{array}$ & Approx. $1 \mathrm{hr}$ & $\begin{array}{l}\text { Per EPA Method } 1 \\
\text { where possible }\end{array}$ & $\begin{array}{l}\mathrm{PM}_{10} \text { sampling train with } \\
\text { cyclones }\end{array}$ & $\begin{array}{l}\text { Isokinetic with the average gas velocity. } \\
\text { SS probes will be used rather than glass } \\
\text { probes for strength }\end{array}$ \\
\hline & $\begin{array}{l}\text { Fume slagging/ } \\
\text { fouling potential }\end{array}$ & EPRI CS-S552 & up to $1 \mathrm{hr}$ & -- & $\begin{array}{l}\text { Water or air-cooled SS } \\
\text { sampling probe }\end{array}$ & $\begin{array}{l}\text { Relative measurement of fume tendency } \\
\text { to adhere to probe }\end{array}$ \\
\hline \multirow[t]{2}{*}{$\begin{array}{l}\text { Sample ports } \\
\text { downstream of } \\
\text { baghouse }\end{array}$} & $\begin{array}{l}\text { Gas velocity, } \\
\text { temperature }\end{array}$ & EPA 1, 2 & $5.30 \mathrm{~min}$ & Per EPA Method 1 & $\begin{array}{l}\text { Sheathed probes as } \\
\text { needed upstream of } \\
\text { ID fan for sealing } \\
\text { in port }\end{array}$ & $\begin{array}{l}\text { Used separately from MMT probe only to } \\
\text { verify installed continuous } V \text { and } T \\
\text { probes }\end{array}$ \\
\hline & Offgas composition & $\begin{array}{l}\text { EPA } 3 A, 6 C, 7 E \\
10,25 A \text {, draft } \\
\text { HCl procedure }\end{array}$ & up to $1 \mathrm{hr}$ traverse & $\begin{array}{l}\text { Minimum } 3 \text { points for } \\
\text { stratification check, then } \\
\text { single-point }\end{array}$ & $\begin{array}{l}\text { Same as above, but the } \\
\mathrm{HCl} \text { probe may be an } \\
\text { air-dilution probe }\end{array}$ & \\
\hline
\end{tabular}


Table 5-4. (continued).

\begin{tabular}{|c|c|c|c|c|c|c|}
\hline $\begin{array}{l}\text { Sample location (port } \\
\text { location) }\end{array}$ & Procedure & Reference & $\begin{array}{c}\text { Sampling/measurement } \\
\text { time }\end{array}$ & Sampling point matrix & Equipment & Comments \\
\hline & $\begin{array}{l}\text { Offgas composition by } \\
\text { gas chromatograph }\end{array}$ & $\begin{array}{l}\text { yMTI operating } \\
\text { manual }\end{array}$ & up to $1 \mathrm{hr}$ & Same as above & Same as above & $\begin{array}{l}\text { Sampling will be continuous at the stack, } \\
\text { and using Tedlar bags for discrete } \\
\text { injection and protection of GC at other } \\
\text { sample locations. Additional gas } \\
\text { scrubbing to remove acid gases, especially } \\
\text { HCl, will be required at sample locations } \\
\text { upstream of the wet scrubber. }\end{array}$ \\
\hline & $\begin{array}{l}\text { Total particulate and } \\
\text { metals }\end{array}$ & $\begin{array}{l}\text { EPA draft Method } \\
29 \text { (MMT) }\end{array}$ & $2-4 \mathrm{hr}$ minimum & Per EPA MMT & & Glass or quartz-lined probe \\
\hline
\end{tabular}


sealed during insertion, sample collection, and removal using a sealing gland around the probe and ball valves on the sample ports similar to the schematic as shown in Figure 5-1.

There may be some cases when using a port momentarily without a tight seal may be necessary. Such cases may include furnace bath depth probing, slag or metal sampling from the bath, bath temperature measurements, and fouling probe removal. At such times the furnace draft will be adjusted to ensure that there will not be gaseous effluents from the furnace through the port.

\subsubsection{High-temperature Measurements}

Accurate gas temperature measurements made in the high temperature furnace plenum, thermal oxidizer inlet and thermal oxidizer outlet locations will require the use of a radiation-shielded suction pyrometer, also called a high velocity temperature (HVT) measuring probe. The general procedure and typical equipment used in these types of measurements is described in EPRI CS-5552. ${ }^{11}$ A schematic of a typical water-cooled suction pyrometer probe is shown in Figure 5-2. The thermocouple tip is shielded from thermal radiation by a metal or ceramic sheath. The gas is drawn through the probe at a sufficiently high velocity so that the convection heat transfer to the thermocouple (TC) is far greater than radiative heat transfer from the sheath or open end of the sheath, and also far greater than conductive heat transfer from the TC to the water-cooled probe. The potential errors from radiative and conductive heat transfer are minimized relative to the convective heat transfer from the gas, enabling the TC to more accurately sense the actual gas temperature.

Velocity measurements in the high temperature regions will be performed using the same procedures specified for velocity measurements in the cooler regions, per EPA Method 2. The high levels of particulate upstream of the baghouse will cause increased plugging that must be monitored and may require frequent removal, especially if the particulate is at or above its melting temperature range. The pitot should be contained in a cooled sheath for sealing in the sample ports and for cooling of the pitot to minimize adhesion of particulate.

Gas composition measurements will require the use of a phase discrimination probe or an in-line cyclone to separate most of the particulate from the sample gas and reduce filter plugging in the sampling system. Phase discrimination (inertial separation of particulate from the sample gas) can be achieved by properly designing a combined suction pyrometer and gas sampling system, so the sample gas for the gas analyzers is a small fraction of the total suction pyrometer flow. An example of such a system is shown in Figure 5-3.

Slagging and fouling in the furnace plenum, thermal oxidizer, and connecting ductwork can occasionally be a significant problem. While fouling was not a problem in the APCS for the FY-93 BWID tests, ${ }^{12}$ there was severe plugging of the furnace outlet duct during the earlier ASME municipal incinerator ash tests. Higher expected offgas temperatures for the planned Phase 2 tests, together with potentially large amounts of entrained soil dust and fume, may result in buildup of accretions in the planned tests under some conditions. The potential for accretions buildup will be evaluated using a stainless steel, cooled fouling probe inserted in the offgas. Using a thermocouple welded to the probe wall, the probe temperature may be set within an approximate range. Accretion buildup on the probe can be monitored over time and compared to other probe and gas temperatures and furnace operating conditions. 


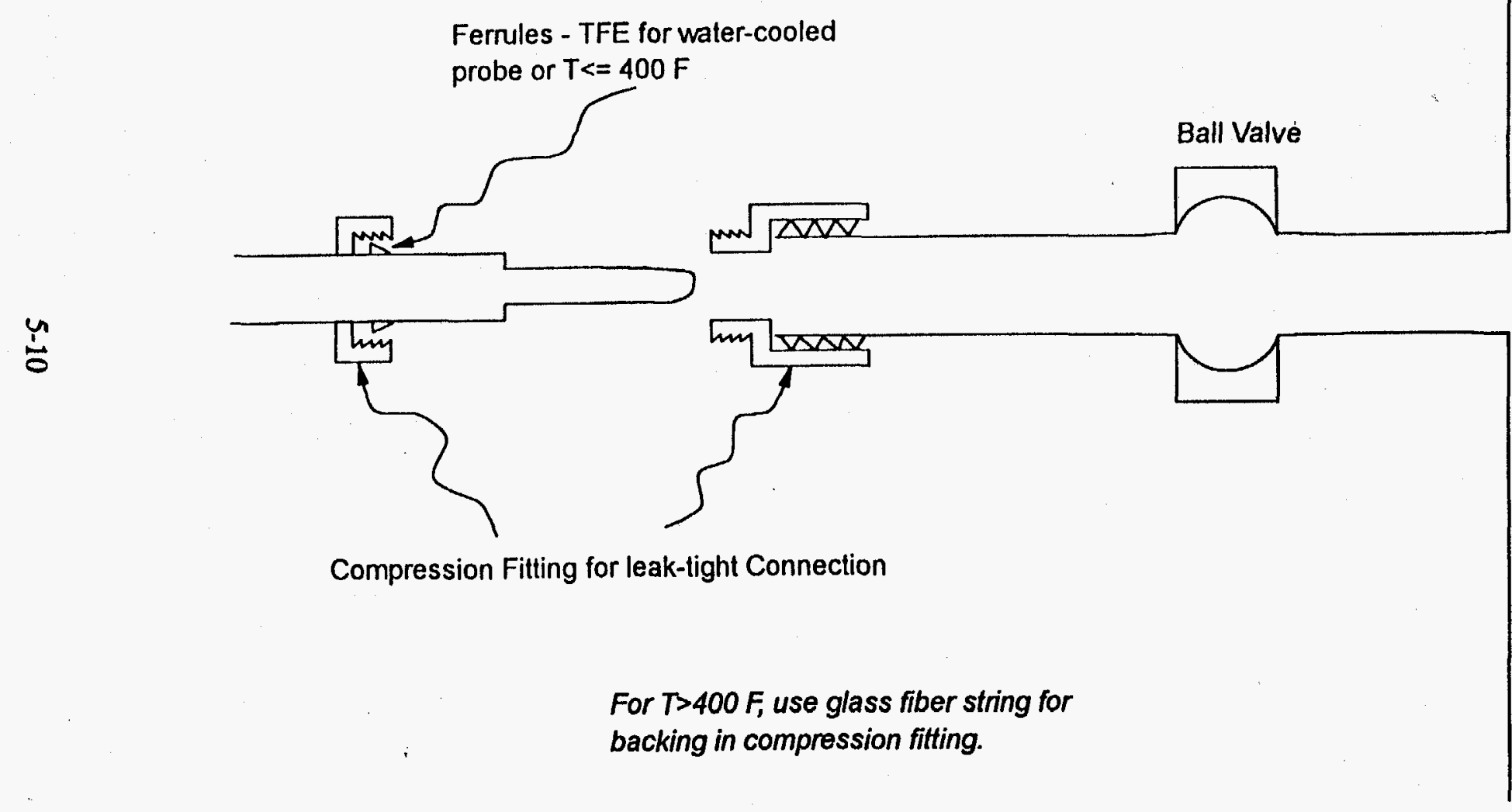

Duct

Figure 5-1. Seal for leak-tight ports during sampling. 


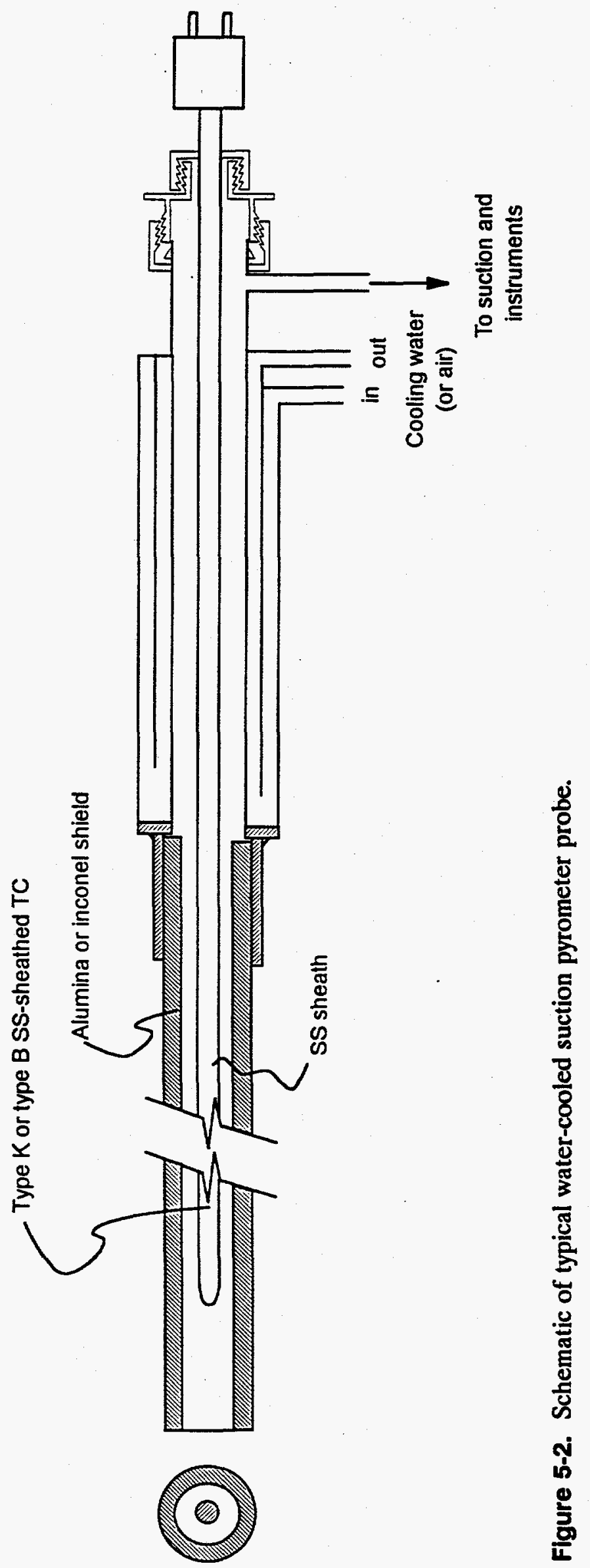




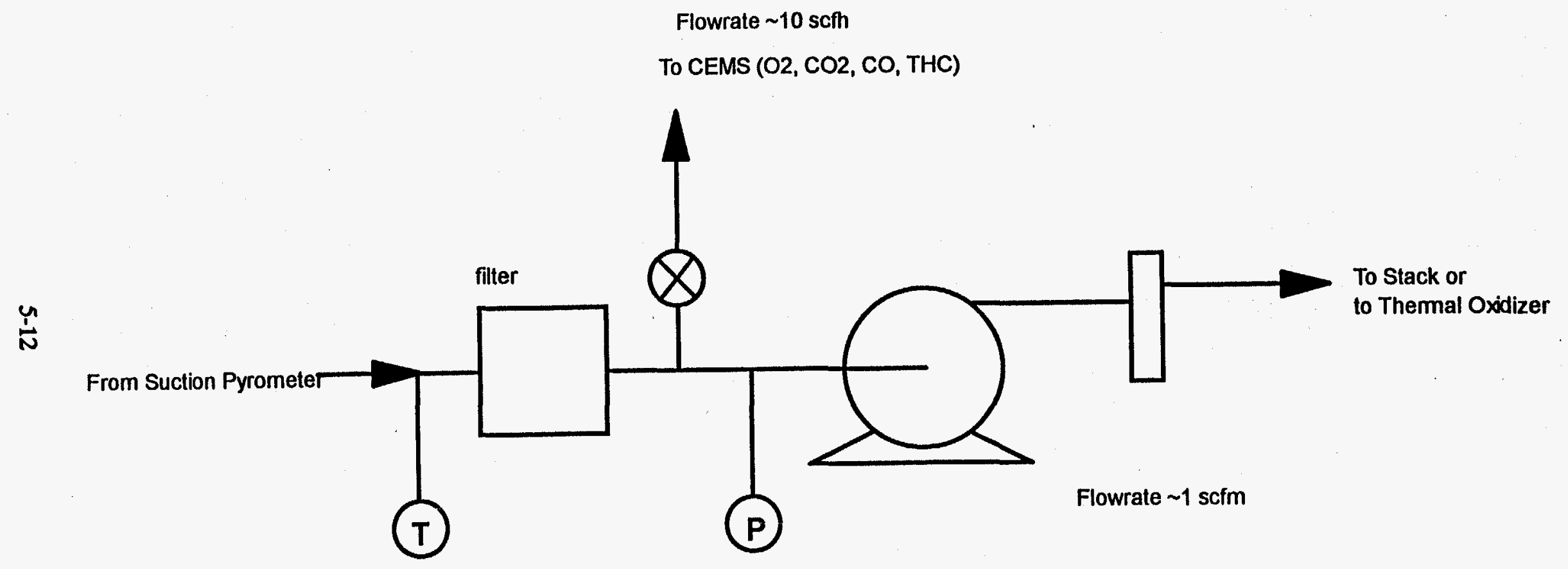

suction.pre

Figure 5-3. CEMS bypass for on the pyrometer suction system. 
Particle size, particulate, and metals will be measured in the high temperature regions using a modified version of the $\mathrm{PM}_{10}$ sampling train. In this procedure, a series of one to three cyclones located on the end of the sample probe are used to separate three different particle size fractions. Sampling at a high rate will reduce the particle size of the fractions so that the particles exiting the last cyclone will be less than $10 \mu$. The sample gas is passed through a filter, and an impinger set containing MMT absorbing solutions, to cool and capture any metals and particulate not captured in the upstream cyclones and the filter. The particulate captured in the cyclones will be mainly entrained dust, while the particulate and gas exiting the third cyclone (and captured in the filter and the impingers) will be enriched in condensed fume from materials volatilized from the melt.

The modified $\mathrm{PM}_{10}$ procedure may have operational difficulties such as buildup of accretions in the cyclones, or damage to the probe and cyclone materials from high temperatures. If this procedure is successful, however, it can provide better differentiation between volatilized materials and entrained feed dust, because separation of the entrained dust particles and the volatilized materials will be done before cooling and condensation of the volatilized materials in the APCS.

If the modified $\mathrm{PM}_{10}$ procedure cannot be successfully used due to the sampling conditions, then one or all of the upfront cyclones will be removed or placed at the back end of the sample probe. This will decrease the ability to differentiate between entrained feed dust and volatilized material.

\subsubsection{Continuous Gaseous Emissions Monitoring Procedures}

The continuous gaseous emissions monitoring will be performed according to Environmental Protection Agency (EPA) continuous monitoring methods. To best characterize the melter emissions and continuously monitor variations in melter performance, sampling will be done in the furnace, at the furnace outlet and at the cooler outlet (upstream of the baghouse) at selected times during the demonstration tests. As discussed in Section 5.3.1, special sampling techniques such as the use of phase discrimination probes and water or air cooling may be required for continuous gas sampling in the high-temperature, particulate laden regions upstream of the spray cooler.

Sample gas will be extracted using a stainless steel sample probe that will be heated or cooled depending on the sample location. Sample collection at the melter outlet may require cooling the sample probe. The sample gas will be filtered and cooled to remove moisture before being introduced to the gas analyzers. Flow to each instrument will be controlled using a sample gas manifold with individual control valves and flowmeters for each instrument. All fittings and sample lines that contact the sample will be constructed of either stainless steel, teflon, or other materials that will not react with the sample gas. Leak checks, bias, residence time, interference, and sampling procedures will be conducted according the EPA applicable reference procedures.

For the purposes of this test program, some variations from the EPA procedures may be utilized that will not significantly compromise the quality of the results. Possible variations include (a) use of less stringent $\pm 2 \%$ calibration gases rather than EPA protocol gases and (b) variations in sample periods or averaging so that sample collection can coincide with melter operating periods.

When continuous monitoring is not necessary, grab samples of offgases at selected locations will be collected for subsequent injection into the CEMS instruments. This will significantly reduce the 
need for replicate CEM systems and provide more flexibility for measuring gas concentrations at various furnace and APCS locations. Grab samples will be collected in reusable Tedlar bags.

\subsubsection{Gas Chromatography Procedures}

Speciated analysis of some gases will be done using a Microtechnologies Inc. (MTI) Q300L gas chromatograph (GC), with three capillary columns and an electrical conductivity detector. Manufacturer information for this $\mathrm{GC}$ is as follows:

$\begin{array}{lll}\begin{array}{ll}\text { Column } \\ \text { Molecular sieve } 10 \mathrm{~m} \text { long }\end{array} & \begin{array}{l}\text { Carrier gas or } \mathrm{Ar} \\ \text { Can detect: }\end{array} & \begin{array}{l}\mathrm{He} \text { (when } \mathrm{Ar} \text { is carrier gas), } \mathrm{H}_{2} \text { (when } \mathrm{Ar} \text { is } \\ \text { carrier gas), } \mathrm{O}_{2}, \mathrm{~N}_{2}, \mathrm{CO} \text { (when He is carrier } \\ \text { gas), } \mathrm{C}_{1}\end{array} \\ \text { Pora Plot Q, } 4 \mathrm{~m} \text { long } & \mathrm{He} \text { or } \mathrm{Ar} & \mathrm{CO}_{2}, \mathrm{C}_{2}-\mathrm{C}_{4} \\ \text { OV-1, } 4 \mathrm{~m} \text { long } & \mathrm{He} \text { or } \mathrm{Ar} & \begin{array}{l}\mathrm{CCl}_{4}, \mathrm{TCE}, \text { several other halogenated } \\ \text { hydrocarbons and volatile organic compounds } \\ \text { (VOCs) }\end{array}\end{array}$

Hydrogen can only be detected when $\mathrm{Ar}$ (not $\mathrm{He}$ ) is the carrier gas because $\mathrm{H}_{2}$ and He have similar retention times on the molecular sieve column. Likewise, $\mathrm{CO}$ can only be detected using $\mathrm{He}$ (not $\mathrm{Ar}$ ) due to similar retention times for $\mathrm{CO}$ and $\mathrm{Ar}$. $\mathrm{NO}, \mathrm{NO}_{2}, \mathrm{HCl}, \mathrm{Cl}_{2}$, and $\mathrm{SO}_{2}$ are too reactive to obtain quantitative measurements except for very high levels of $\mathrm{NO}$ and $\mathrm{SO}_{2}$. It may be possible to detect aldehydes on the OV-1 column. High levels of acid gases, especially $\mathrm{HCl}$ but also $\mathrm{SO}_{2}$ and $\mathrm{NO}_{2}$, can rapidly damage components of the $\mathrm{GC}$, and so must be scrubbed out of the gas for all of the sample locations except downstream of the wet scrubber. The sample gas at all locations must be filtered and cooled to below ambient temperature to prevent particulate or water droplets in the GC injector, even though there is a backup Genie filter in the GC inlet. Particulate or water droplets in the injector will invalidate results and may damage the GC.

\subsubsection{Metals and Particulate Sampling Procedures}

Gaseous and particulate metals emissions in the offgas will be determined using the EPA MMT (Draft EPA Method 29, October 29, 1990). This procedure is modeled after the EPA Method 5 procedure (40 CFR 60, Appendix A, 1990) for determining total particulate emissions from stationary sources. Using the Draft Method 29 procedure, an offgas sample is collected isokinetically from the offgas stream to obtain the most representative gaseous and entrained particulate sample. Using a heated, glass-lined sample probe with a sharp-edged glass nozzle, the duct is traversed during sample collection to minimize the effects of velocity and concentration stratification. The sample is passed through a filter heated to $250 \pm 25^{\circ} \mathrm{F}$, which is used to capture particulate or aerosol material at that temperature. The filtered sample is then passed through a series of glass impingers that contain absorbing solution for capturing metals and other materials. The last impinger contains silica gel for complete drying of the gas sample. The impingers are maintained in an ice bath so that the gas sample is rapidly cooled, and the temperature of gas leaving the last (silica gel) impinger is $68^{\circ} \mathrm{F}$ or 
less. The volume and sample rate of filtered, dried sample gas is controlled and monitored using a calibrated dry gas meter, sharp-edged orifice, and sample pump.

Following sample collection, the sample is quantitatively recovered from the inside of the nozzle and sample probe, filter housing, connecting glassware, and impingers. The front half of the sample train (from the probe nozzle to the filter) will be gravimetrically analyzed to determine the solid particulate levels in the offgas. The front and back halves (downstream of the filter, including the impinger catch) will be analyzed for the target metals and surrogate radionuclides by ion-coupled argon plasma spectroscopy (ICAP) or by graphite furnace atomic absorption spectroscopy (GFAAS). If mercury is one of the target metals, cold-vapor atomic absorption spectroscopy (CVAAS) is used.

Chemical reagents of the minimum purity and specifications identified in the Draft EPA Method 29 procedures will be used for all sampling and analysis activities. All materials that contact the sample will be glass or other nonmetallic, noncontaminating material. All connections will be leak free, ground glass fittings, or other noncontaminating materials. All glassware and sample containers will be carefully cleaned using the specified metals-free reagents and dried in a dust-free environment after cleaning.

All sampling and analysis procedures will be performed in accordance with the specifications in the draft method. These procedures include sample train preparation, calibration, reagent preparation, leak checks, train operation, and sample recovery, preparation, and analysis. QA/quality control (QC) procedures will also be performed in accordance with requirements specified in the EPA Draft Method 29, EPA 6010, or EPA 7000-series procedures.

Variations from the standard Draft EPA Method 29 procedure may include (a) elimination of the impingers that contain absorbing solution (acidified potassium permanganate) for capturing mercury, (b) elimination of mercury analysis by CVAAS, (c) inclusion of gravimetric analysis of the train front half for solid particulate determination, (d) combination of the train front and back half metals analysis for reduced analysis cost, (e) use of a short stainless steel probe instead of a glass probe for combination. with the in-stack $\mathrm{PM}_{10}$ particle sizing procedure, and (f) single-point sampling (MMT-SP) when deemed necessary to expedite sample collection. Any other modifications to the sampling and analysis procedures that are necessary to meet the project objectives will be made to ensure data accuracy and representativeness of the operating conditions during the test periods. All changes will be carefully documented and justified with all available support information.

\subsubsection{Offgas Sampling Team}

The offgas and particulate sample collection and analysis performed during the onsite test periods includes sample collection and measurements at up to several locations in the furnace and APCS. The sampling conditions upstream of the spray cooler will be most severe, being in the proximity of high-temperature equipment, near the gas burners and the furnace power system, and having high temperature and particulate laden offgas. Sampling downstream of the spray cooler will also require making exacting measurements of offgas and particulate. These activities will require up to three experienced personnel. The expected duties for these personnel are described below. 
Person

1

2,3

4,5

6

7

Activity

Sample team leader, QA overview, sampling and process coordination, and gaseous and metals train data analysis-N.R. Soelberg of LITCO

Sample collection and measurements upstream of the spray cooler, including suction pyrometer temperature measurements, velocity measurements, CEMS sampling, and $\mathrm{PM}_{10}$ sampling for selected test conditions $\rightarrow$ subcontracted test personnel

Sample collection and measurements downstream of the spray cooler, including temperature and velocity measurements, CEMS sampling, and MMT sampling for selected test conditions-subcontracted test personnel

Continuous monitoring and CEMS data analysis-subcontracted test personnel

GC operations and analysis-Del Baker of USBM

The above specified personnel will be required for test conditions when particulate sampling is performed. Fewer personnel will be required for test conditions when only temperature, velocity, and gaseous measurements are necessary.

The recommended minimum amounts and types of test equipment for the fume and offgas sampling includes

- Suction pyrometer, velocity and CEMS measurements upstream of the spray cooler-Water-cooled, sheathed probe and sampling system that includes the suction pyrometer, an S-type pitot, and CEMS bypass. The CEMS bypass system should have the necessary filtration and sample conditioning equipment to tolerate the high-temperature, particulate-laden offgas and protect the downstream sample equipment and instrumentation during continuous operation.

- Separate suction pyrometer, pitot and gas sampling probes, sheathed for sealing in sample ports, for separate measurements and sampling as needed

- $\quad \mathrm{PM}_{10}$ sample collection-At least two control boxes (one with a spare), two and two each umbilicals, heated filter boxes, impinger boxes, sample probes with cyclones, nozzles, and miscellaneous spare parts such as fittings, tubing, thermocouples, and glassware.

- Velocity and temperature measurements downstream of spray cooler-uncooled, sheathed TC and pitot for leak-tight sampling through ports

- CEMS sampling downstream of spray cooler-uncooled, filter-tipped probes for both continuous and Tedlar bag sampling. The Tedlar bag sampling system should include a separate pump in a leak-tight, portable sampling apparatus.

- CEMS instrumentation-one complete system, with the sampling system valved to be able to readily switch between continuous sampling through sample lines upstream or downstream of the spray cooler, or to sample from Tedlar bags. The instrumentation 
should include (a) the required analyzers in a temperature-controlled environment, (b) a conditioning system, (c) zero, mid-range and high-range calibration gases (certified $\pm 2 \%$ or better), (d) chart recorder, and (e) optional computer data acquisition system.

- MMT sample collection-At least two each (one with a spare) control boxes, umbilicals, heated filter boxes, impinger boxes, heated, glass-lined sample probes with nozzles, glassware, filter housings, and miscellaneous spare parts such as fittings, tubing, and thermocouples.

- GC instrumentation-MTI GC with carrier gases and calibration gases. The GC inlet should be valved to continuously receive conditioned gas from the stack sample location, and also be fitted with a port for injecting gas from Tedlar bags.

- Portable Tedlar bag sampling system-complete with an uncooled sample probe, a filter, a gas cooler and scrubber, and a sample pump, with fittings for easy Tedlar bag handling. 


\section{DOCUMENT CONTROL}

Control of project documents, test data, and project files is important for this cooperative program, which is sponsored and performed by different entities. The USBM document control system is being used for test records and system configuration control. Where applicable, compliance with the LITCO standards for BWID issued documents ${ }^{13}$ will be followed. Document generation and control procedures will provide necessary recordkeeping documentation for BWID.

\subsection{Data Documentation, Control, and Storage}

Document control for test data will begin with the raw data sheets and sample analysis data. These raw data records will be maintained in project files at the USBM by those generating the raw data. The process operating, monitoring, and process sample collection data will be documented and controlled by the USBM. Hard copies such as the hand-recorded data logs will be filed by the USBM lead personnel, while electronic copies of the data logger output will be filed on 3.5-in. disks and on the computer hard-drive. Copies of both the hard-copies and electronic files will also be provided to LITCO personnel.

The offgas sampling and analysis data will be controlled by LITCO, but copies of both electronic files and hard copies will also be maintained by the subcontracted testing firm. Engineering drawings and configuration control for the melter test system and facility will be controlled by USBM, but copies will be provided to LITCO.

\subsection{Test Plan Change Control}

This test plan has been prepared by LITCO with contributions from USBM. The test plan is the primary test project guidance document for the Phase 2 test program. The test plan is a controlled document of LITCO. The USBM will generate specific test procedures as needed within the logical framework of this test plan.

Changes may be made to the test plan before or during the demonstration test by the cognizant test personnel. Any necessary changes may be made before the demonstration tests by agreement between the LITCO Principal Investigators and the ALRCMelter Facility Project Manager. Changes made during the demonstration tests may be made by responsible onsite LTTCO and ALRC test supervisory personnel. Changes will only be made using sound engineering judgement to ensure the highest possible data quality within available funding and safe operation, in the event of circumstances that cause deviations from the test plan. While it is planned to complete the full scope of test conditions, sampling and analysis described in this test plan, there are several potential circumstances that may dictate changes to the test plan prior to or during the demonstration tests. These circumstances include (a) insufficient BWID funding, (b) information provided by other programs such as the Idaho Waste Processing Facility team, and (c) results of the planned shakedown tests. Any changes will be documented in test logs. 


\subsection{Document Control for Other Documents}

Other documents will include the (a) quick look field data test report summarizing activities of the demonstration test, and (b) the evaluation report prepared by ALRC and LITCO. All project notes, correspondence, and other project detailed files will become part of the project files controlled by LITCO and USBM. 


\section{ANALYTICAL METHODS}

The sample analysis matrix for the demonstration test samples is shown in Table 7-1. All of the sample analysis will be conducted at the ALRC or another experienced laboratory, selected for those few analyses the ALRC laboratory is not equipped to perform. The most critical analyses are summarized in Table 7-2. These analyses include those performed for the process samples and offgas samples. Standard EPA-promulgated methods are used where possible. Some analytes, such as the TRU surrogates, are not specifically included in the cited methods. However, the most applicable methods will be applied where possible to achieve the most accurate results, and the laboratory will base procedures on results obtained from similar analyses on similar samples performed in the Phase 1 test program. QA activities specified by these methods typically include spikes, duplicates, and splits. However, analysis standards are not readily available for some elements, so such QA activities will be performed only where standards are available. 
Table 7-1. Sample analysis matrix.

\begin{tabular}{|c|c|c|c|c|c|c|c|c|c|}
\hline \multirow[b]{2}{*}{$\begin{array}{l}\text { Test } \\
\text { no. }\end{array}$} & \multirow[b]{2}{*}{ Test objective } & \multirow[b]{2}{*}{ Sample type } & \multirow[b]{2}{*}{$\begin{array}{c}\text { Full } \\
\text { elemental }^{\mathrm{a}}\end{array}$} & \multirow[b]{2}{*}{ formers $^{\text {Slag }}$} & \multirow[b]{2}{*}{$\begin{array}{c}\text { Partial } \\
\text { elemental }^{c}\end{array}$} & \multicolumn{3}{|c|}{ Sample Analyes } & \multirow[b]{2}{*}{$\begin{array}{l}\text { SEMI } \\
\text { Micro- } \\
\text { probe }\end{array}$} \\
\hline & & & & & & $\mathrm{Fe}^{+2} / \mathrm{Fe}^{+3}$ & $X_{R D}^{d}$ & $\begin{array}{l}\text { Leach- } \\
\text { abilitye }\end{array}$ & \\
\hline \multirow[t]{9}{*}{ RDF-0 } & \multirow[t]{9}{*}{$\begin{array}{l}\text { RDF bottom ash } \\
\text { demonstration }\end{array}$} & Slag & $\begin{array}{l}2 \text { (last } \\
2 \text { pots) }\end{array}$ & 5 & & $\begin{array}{l}2 \text { (last } \\
2 \text { pots) }\end{array}$ & $\begin{array}{l}2 \text { (last } \\
2 \text { pots) }\end{array}$ & $\begin{array}{l}2 \text { (nst } \\
2 \text { pots) }\end{array}$ & \\
\hline & & Metal & 1 & & & & & & \\
\hline & & TO catch & 1 & & & & & & 1 \\
\hline & & Cyclone catch & 1 & & & & & & 1 \\
\hline & & BH catch & 1 & & & & & & 1 \\
\hline & & APCS comp & & & & 1 & & 1 & \\
\hline & & Scrubber BD & & & 1 & & & & \\
\hline & & MMT FH & & & 1 & & & & \\
\hline & & MMT BH & & & 1 & & & . & . \\
\hline \multirow[t]{9}{*}{ RDF-10A } & \multirow{9}{*}{$\begin{array}{l}\text { RDF bottom ash } \\
\text { w/asbestos } \\
\text { surrogate }\end{array}$} & Slag & 2 & 5 & & 2 & 2 & 2 & \\
\hline & & Metal & 1 & & & & & & \\
\hline & & TO catch & 1 & & & & & & 1 \\
\hline & & Cyclone catch & 1 & & & & & & 1 \\
\hline & & BH catch & 1 & & & & & & 1 \\
\hline & & APCS comp & & & & 1 & & 1 & \\
\hline & & Scrubber BD & & & 1 & & & & \\
\hline & & MMT FH & & & 2 & & & & \\
\hline & & MMT BH & & & 2 & & & & \\
\hline \multirow[t]{4}{*}{ Nom-90 } & \multirow{4}{*}{$\begin{array}{l}\text { Stepwise increase } \\
\text { of nominal waste } \\
\text { mixture in soil }\end{array}$} & Slag & 2 & 5 & & & & & \\
\hline & & TO catch & 1 & & & & & & \\
\hline & & Cyclone catch & 1 & & & & & & \\
\hline & & BH calch & 1 & & & & & & \\
\hline \multirow[t]{4}{*}{ Nom-80-1 } & \multirow{4}{*}{$\begin{array}{l}\text { Stepwise increase } \\
\text { of nominal waste } \\
\text { mixture in soil } \\
\text { (Same sampling } \\
\text { and analysis } \\
\text { matrix that Nom- } \\
90 \text { has) }\end{array}$} & Slag & 2 & 5 & & & & & \\
\hline & & To catch & 1 & & & & & & \\
\hline & & Cycione catch & 1 & & & & & & \\
\hline & & BH catch & 1 & & & & & & \\
\hline
\end{tabular}


Table 7-1. (continued).

\begin{tabular}{|c|c|c|c|c|c|c|c|c|c|}
\hline \multirow[b]{2}{*}{$\begin{array}{l}\text { Test } \\
\text { no. }\end{array}$} & \multirow[b]{2}{*}{ Test objective } & \multirow[b]{2}{*}{ Sample type } & \multirow[b]{2}{*}{$\begin{array}{c}\text { Full } \\
\text { elementa }^{\mathrm{a}}\end{array}$} & \multirow[b]{2}{*}{ formers $^{b}$} & \multirow[b]{2}{*}{$\begin{array}{c}\text { Partial } \\
\text { elemental }\end{array}$} & \multicolumn{4}{|c|}{ Sample Analyses } \\
\hline & & & & & & $\mathrm{Fe}^{+2} / \mathrm{Fe}^{+3}$ & $\mathrm{XRD}^{\mathbf{d}}$ & $\begin{array}{l}\text { Leach- } \\
\text { abilitye }\end{array}$ & $\begin{array}{l}\text { SEMV } \\
\text { Micro- } \\
\text { probe }\end{array}$ \\
\hline \multirow[t]{9}{*}{ Nom-80-2 } & \multirow{9}{*}{$\begin{array}{l}\text { System } \\
\text { performance } \\
\text { cvaluation }\end{array}$} & Slag & 2 & 5 & & 2 & 2 & 2 & \\
\hline & & Metal & 1 & & & & & & \\
\hline & & TO catch & 1 & & & & & & 1 \\
\hline & & Cyclone catch & 1 & & & & & & 1 \\
\hline & & BH catch & 1 & & & & & & 1 \\
\hline & & APCS comp & & & & 1 & & 1 & \\
\hline & & MMT FH & & & 1 & & & & \\
\hline & & MMT ВH & & & 1 & & & & \\
\hline & & Scrubber BD & & & 1 & & & & \\
\hline \multirow[t]{5}{*}{ Nom-70 } & \multirow{5}{*}{$\begin{array}{l}\text { Stepwise increase } \\
\text { of nominal waste } \\
\text { mixture in soil } \\
\text { (Same sampling } \\
\text { and analysis } \\
\text { matrix that Nom- } \\
90-1 \text { has) }\end{array}$} & Slag & 2 & 5 & & & & & \\
\hline & & Metal & 1 & & & & & & \\
\hline & & TO catch & 1 & & & & & & \\
\hline & & Cyclone catch & 1 & & & & & & \\
\hline & & BH catch & 1 & & & & & & \\
\hline \multirow[t]{9}{*}{ Nom-60-1 } & \multirow{9}{*}{$\begin{array}{l}\text { Stepwise increase } \\
\text { of nominal waste, } \\
\text { lower power } \\
\text { density and } \\
\text { feedrate }\end{array}$} & Slag & 2 & 5 & & 2 & 2 & 2 & \\
\hline & & Metal & & & & & & & \\
\hline & & TO catch & 1 & & & & & & 1 \\
\hline & & Cyclone catch & 1 & & & & & & 1 \\
\hline & & BH catch & 1 & & & & & & 1 \\
\hline & & APCS comp & & & & 1 & & 1 & \\
\hline & & MMT FH & & & 1 & & & & \\
\hline & & MMT BH & & & 1 & & & & \\
\hline & & Scrubber BD & & & 1 & & & & \\
\hline \multirow[t]{9}{*}{ Nom-60-2 } & \multirow{9}{*}{$\begin{array}{l}\text { Added solid } \\
\text { oxidant in feed }\end{array}$} & Slag & 2 & 5 & & 2 & 2 & 2 & \\
\hline & & Metal & 1 & & & & & & \\
\hline & & TO catch & 1 & & & & & & 1 \\
\hline & & Cyclone catch & 1 & & & & & & 1 \\
\hline & & BH catch & 1 & & & & & & 1 \\
\hline & & APCS comp & & & & 1 & & 1 & \\
\hline & & MMT FH & & & 1 & & & & \\
\hline & & MMT BH & & & 1 & & & & \\
\hline & & Scrubber BD & & & 1 & & & & \\
\hline
\end{tabular}


Table 7-1. (continued).

\begin{tabular}{|c|c|c|c|c|c|c|c|c|c|}
\hline \multirow[b]{2}{*}{$\begin{array}{l}\text { Test } \\
\text { no. }\end{array}$} & \multirow[b]{2}{*}{ Teat objective } & \multirow[b]{2}{*}{ Sample type } & \multirow[b]{2}{*}{ elemental $^{\mathrm{a}}$} & \multirow[b]{2}{*}{$\underset{\text { formers }}{\text { Slag }}$} & \multirow[b]{2}{*}{$\underset{\text { elemental }}{\text { Partial }}$} & \multicolumn{4}{|c|}{ Sample Analyses } \\
\hline & & & & & & $\mathrm{Fe}^{+2} / \mathrm{Fe}^{+3}$ & $X R D^{d}$ & $\begin{array}{l}\text { Leach- } \\
\text { abilitye }\end{array}$ & $\begin{array}{l}\text { SEIM/ } \\
\text { Micro- } \\
\text { probef }\end{array}$ \\
\hline \multirow[t]{8}{*}{ Nom-60-3 } & \multirow{8}{*}{$\begin{array}{l}\text { Added gir thru } \\
\text { lance for } \\
\text { oxidizing metal }\end{array}$} & Slag & 2 & 5 & & 2 & 2 & 2 & \\
\hline & & TO catch & 1 & & & & & & 1 \\
\hline & & Cyclone catch & 1 & & & & & & 1 \\
\hline & & BH catch & 1 & & & & & & 1 \\
\hline & & APCS comp & & & & 1 & & 1 & \\
\hline & & MMT FH & & & 2 & & & & \\
\hline & & MMT BH & & & 2 & & & & \\
\hline & & Scrubber BD & & & 1 & & & & \\
\hline \multirow[t]{9}{*}{ Nom-60-4 } & \multirow{9}{*}{$\begin{array}{l}\text { Operate without } \\
\text { cold top }\end{array}$} & Slag & 2 & 5 & & 2 & 2 & 2 & \\
\hline & & Metal & 1 & & & & & & \\
\hline & & TO catch & 1 & & & & & & 1 \\
\hline & & Cyclone catch & 1 & & & & & & 1 \\
\hline & & BH catch & 1 & & & & & & 1 \\
\hline & & APCS comp & & & & 1 & & 1 & \\
\hline & & Scrubber BD & & & 1 & & & & \\
\hline & & MMT FH & & & 2 & & & & \\
\hline & & MMT BH & & & 2 & & & & \\
\hline \multicolumn{2}{|c|}{ Total Samples } & & 54 & 50 & 27 & 19 & 14 & 21 & 21 \\
\hline
\end{tabular}

a. Full elemental-full set metais (18 metals-Al, $\mathrm{As}, \mathrm{Ba}, \mathrm{Ca}, \mathrm{Ce}, \mathrm{Cr}, \mathrm{Cs}, \mathrm{Cu}, \mathrm{Fe}, \mathrm{K}, \mathrm{Mg}, \mathrm{Na}, \mathrm{Ni}, \mathrm{Pb}, \mathrm{Si}, \mathrm{Ti}, \mathrm{Zn}$, and $\mathrm{Zr}$ ) plus $\mathrm{P}, \mathrm{S}, \mathrm{C}$, and $\mathrm{Cl}$.

b. Slag formers include $\mathrm{Al}, \mathrm{Ca}, \mathrm{Fe}, \mathrm{K}, \mathrm{Mg}, \mathrm{Na}$, and $\mathrm{Si}$.

c. Partial elemental (15 metals)-Al, As, Ba, Ca, Ce, Cr, Cs, Cu, K, Mg, Na, Ni, Pb, Si, Zn.d. XRD-X-ray diffraction.

d. XRD-X-ray diffraction.

e. Leachability tests include both toxicity characteristic leachability procedure (TCLP) and PCT-1.

f. SEM-Scanning electron micrograph, and includes determination of particle size distribution. Other potential alternative analyses include $x$-ray fluorescence and optical microscopy. 
Table 7-2. Sample analysis procedures.

\begin{tabular}{|c|c|c|c|c|c|c|}
\hline Applicable samples/materials & Analytes & Method & Analysis procedure & Detection limits & $\begin{array}{l}\text { Handling and } \\
\text { preparation }\end{array}$ & Method highlights \\
\hline \multirow[t]{12}{*}{$\begin{array}{l}\text { All process samples, including slag } \\
\text { samples, metal samples, and APCS } \\
\text { solid samples (catches from the } \\
\text { thermal oxidizer, spray dryer, and } \\
\text { baghouse) }\end{array}$} & $\begin{array}{l}\text { Full set } \\
\text { metals }\end{array}$ & $\begin{array}{l}\text { SW846 } 6010 \text { or } \\
7000 \text { Series }(\mathrm{Hg} \\
\text { by } 7470)\end{array}$ & $\begin{array}{l}\text { ICAP }^{b}, \text { GFAAS }^{c} \text {, or } \\
\text { CVAAS }^{d}\end{array}$ & $\begin{array}{l}\text { Varies, generally } \\
1-10 \mathrm{mg} / \mathrm{kg}\end{array}$ & $\begin{array}{l}100 \mathrm{mg}-1 \mathrm{~g} \\
\text { sample } \\
\text { digestions } \\
\text { with } \mathrm{HF} \text {, } \\
\mathrm{HC}, \mathrm{HNO}_{3} \\
\text { per USBM } \\
\text { ALRC } \\
\text { procedure }\end{array}$ & $\begin{array}{l}\text { ICAP for most analytes. Cs by GFAAS } \\
\text { using standard additions, no spike. Hg by } \\
\text { CVAAS. Following ICAP analysis, data } \\
\text { will be evaluated to determine } \\
\text { applicability of GFAAS for some anatytes } \\
\text { to improve detection limits }\end{array}$ \\
\hline & $\mathbf{P}$ & $\begin{array}{l}\text { AOAC method } \\
\text { total } \\
\text { phosphorous } \\
\text { determination }\end{array}$ & $\begin{array}{l}\text { Digestion/ } \\
\text { precipitation/ } \\
\text { gravimetry }\end{array}$ & Estimate $\mathbf{m g} / \mathrm{kg}$ & 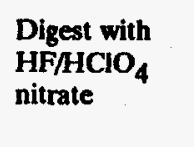 & $\begin{array}{l}\text { AOAC method will be modified slightly } \\
\text { per USBM ALRC experience }\end{array}$ \\
\hline & $\mathbf{s}$ & LECO CS144 & $\begin{array}{l}\text { LECO CS144 gas } \\
\text { analyzer }\end{array}$ & 5 ppm by wt. & -- & $\begin{array}{l}\text { High-temperature oxidation and infrared } \\
\text { gas analysis }\end{array}$ \\
\hline & Total C & LECO CS144 & $\begin{array}{l}\text { LECO CS } 144 \text { gas } \\
\text { analyzer }\end{array}$ & 5 ppm by wt. & - & $\begin{array}{l}\text { High-lemperature oxidation and infrared } \\
\text { gas analysis }\end{array}$ \\
\hline & $\mathbf{C l}$ & $\begin{array}{l}\text { USBM ALRC } \\
\text { analysis }\end{array}$ & Ion chromatography & $2 \mathrm{mg} / \mathrm{kg}$ & $\begin{array}{l}\text { Digest with } \\
\mathrm{H}_{2} \mathrm{SO}_{4}\end{array}$ & > 24-h digestions, followed by IC analysis \\
\hline & $\begin{array}{l}\text { PCT-1 } \\
\text { leachability }\end{array}$ & PCT-1 & Not available & Not available & Not available & $\begin{array}{l}\text { Basic digestion followed by analysis of } \\
\text { liquor }\end{array}$ \\
\hline & $\begin{array}{l}\text { TCLP } \\
\text { leachability }\end{array}$ & $\begin{array}{l}\text { TCLP', } 40 \text { CFR } \\
\text { 261, App. II }\end{array}$ & $\begin{array}{l}\text { Size-reduce, acid } \\
\text { extraction, SW846 } \\
\text { analysis }\end{array}$ & $\begin{array}{l}\text { Varies, generally } \\
1-10 \mathrm{ng} / \mathrm{mL}\end{array}$ & $\begin{array}{l}\text { Grind to size } \\
<9.5 \mathrm{~mm}\end{array}$ & $\begin{array}{l}\text { Mild acid digestion followed by analyais of } \\
\text { liquor by SWS } 46 \text { methods }\end{array}$ \\
\hline & Total Fe & $\begin{array}{l}\text { USBM IC } 9240 \\
\text { Total Iop } \\
\text { Analysis }\end{array}$ & $\begin{array}{l}\text { Oxidation by potassium } \\
\text { dichromate/dichromate } \\
\text { titration }\end{array}$ & $1-10 \mathrm{mg} / \mathrm{kg}$ & -- & $\begin{array}{l}\text { Better results than GFAAS when iron } \\
\text { level }>2 \mathrm{wt} \%\end{array}$ \\
\hline & $\mathrm{Fe}^{+2} / \mathrm{Fe}^{+3}$ & $\begin{array}{l}\text { USBM IC } 9240 \\
\text { Ferrous Iron } \\
\text { Analysis }^{\mathrm{h}}\end{array}$ & Ferrous iron titration & $0.1-1 w \%$ & $\begin{array}{l}\text { Digest using, } \\
\text { vanadium }+5 \text {, } \\
\mathrm{H}_{2} \mathrm{SO}_{4} / \mathrm{HF}\end{array}$ & Wet chemistry technique \\
\hline & $\mathbf{S i}$ & $\begin{array}{l}\text { USBM IC } 9240 \\
\text { Gravimetric } \\
\text { Silica } \\
\text { Determination }^{\mathrm{h}}\end{array}$ & $\begin{array}{l}\text { Gravimetric } \\
\text { dehydration }\end{array}$ & $1-10 \mathrm{mg} / \mathrm{kg}$ & -- & $\begin{array}{l}\text { Silicic acid is dehydrated using sulfuric } \\
\text { and perchloric acids, } \mathrm{HF} \text { addition, } \mathrm{SiF}_{4} \\
\text { volatilization, and gravimetric analysis }\end{array}$ \\
\hline & $\begin{array}{l}\text { Physical } \\
\text { character- } \\
\text { istics, } \\
\text { particle size }\end{array}$ & $\begin{array}{l}\text { Scanning } \\
\text { electron } \\
\text { microscope }\end{array}$ & --- & $\cdots$ &.- & $\begin{array}{l}\text { Subjective analysis by experienced SEM, } \\
\text { technician }\end{array}$ \\
\hline & $\begin{array}{l}\text { Chemical } \\
\text { composition } \\
\text { of specific }\end{array}$ & Microprobe & $\cdots$ & $0.1 w t \%$ & $\ldots$ & $\begin{array}{l}\text { Subjective analysis by experienced SEM } \\
\text { technician }\end{array}$ \\
\hline
\end{tabular}


Table 7-2. (continued).

\begin{tabular}{|c|c|c|c|c|c|c|}
\hline Applicable samples/materials & Analytes & Method & Analysis procedure & Detection limits & $\begin{array}{l}\text { Handling and } \\
\text { preparation }\end{array}$ & Method highlights \\
\hline . & $\begin{array}{l}\text { Crystalline } \\
\text { structures }\end{array}$ & $\mathbf{X}$-ray diffraction & -- & $3-5 w t \%$ & $\cdots$ & \\
\hline $\begin{array}{l}\text { Process material (feed barrels, slag } \\
\text { pots, baghouse barrels, electrode } \\
\text { weights, etc) }\end{array}$ & Weight & $\begin{array}{l}\text { Magna-View } \\
\text { Floor Scale } \\
\text { (Howe } \\
\text { Richardson } \\
\text { Scale Co.) }\end{array}$ & Gravimetric & $\begin{array}{l}2 \text { ib graduations; } 0.00 \\
\text { \% calibrated error }\end{array}$ & & \\
\hline APCS catches & Weight & $\begin{array}{l}\text { Fairbanks-Morse } \\
\text { Springless Scale }\end{array}$ & Gravimetric & $\begin{array}{l}2 \text { oz. graduations; } \\
0.08 \text { oz/lb calibrated } \\
\text { error }\end{array}$ & - & $\begin{array}{l}\text { Quantitative determination of all of the } \\
\text { fume deposited material recovered }\end{array}$ \\
\hline $\begin{array}{l}\text { EPA } 29 \text { samples (probe acetone } \\
\text { and acid catches, filter catches, and } \\
\text { impinger catches) }\end{array}$ & $\begin{array}{l}\text { Partial set } \\
\text { metais }\end{array}$ & $\begin{array}{l}\text { EPA Draft } \\
\text { Method } 29 \\
\text { (SW846 } 6010 \text { or } \\
7000 \text { Series, } \mathrm{Hg}_{\mathrm{g}} \\
\text { by 7470) }\end{array}$ & $\begin{array}{l}\text { ICAP, GFAAS, or } \\
\text { CVAAS }\end{array}$ & $\begin{array}{l}\text { Varies, generally } \\
1-10 \mathrm{ng} / \mathrm{mL}\end{array}$ & $\begin{array}{l}\text { EPA Method } \\
\text { 29-specified } \\
\text { procedures }\end{array}$ & $\begin{array}{l}\text { Metals analysis is done following gravi- } \\
\text { metric analysis on the probe acetone } \\
\text { catches and filter catches. ICAP for most } \\
\text { analytes. Cs by GFAAS using standard } \\
\text { additions, no spike. Following ICAP } \\
\text { analysis, data will be evaluated to } \\
\text { determine applicability of GFAAS for } \\
\text { some analytes to improve detection limits }\end{array}$ \\
\hline $\begin{array}{l}\text { EPA } 29 \text { samples (probe acetone } \\
\text { catches and filter catches) }\end{array}$ & Weight & EPA Method 5 & Gravimetric & Per EPA Method 5 & $\begin{array}{l}\text { Per EPA } \\
\text { Method } 5\end{array}$ & $\begin{array}{l}\text { Following gravimetric analysis, recombine } \\
\text { with acid probe catch and impinger catch } \\
\text { for metals analysis }\end{array}$ \\
\hline
\end{tabular}

a. Full set metals (18 metals)-Al, As, Ba, $\mathrm{Ca}, \mathrm{Ce}, \mathrm{Cr}, \mathrm{Cs}, \mathrm{Cu}, \mathrm{Fe}, \mathrm{K}, \mathrm{Mg}, \mathrm{Na}, \mathrm{Ni}, \mathrm{Pb}, \mathrm{Si}, \mathrm{Ti}, \mathrm{Zn}$, and $\mathrm{Zr}$.

b. ICAP-lon-coupled argon plasma spectroecopy; using 1-g sample in $100 \mathrm{~mL}$ digestion solution for process samples.

c. GFAAS-Graphite furnace atomic absorption spectroscopy, using $200 \mathrm{mg}$ sample in $\mathrm{HNO}_{3} \mathrm{HF}$ solution for process samples.

d. CVAAS-Cold vapor atomic absorption spectroscopy.

e. All vitrified samples will be digested using the USBM ALRC "Method for Dissolution of Vitrified Samples," August 19, 1992.

f. Official Methods of Analysis of the Association of Official Agricultural Chemists, 10th Edition, 1965, pages 11-13.

g. TCLP-Toxicity characteristic leachability procedure.

h. D. A. Baker and J. W. Sigle, "Methods for the Anatysis of Mineral Chromites and Ferrochrome Slag," Bureau of Mines Information Circular, IC $9240,1990$.

i. Partial Set Metals (15 metals)-Al, As, Ba, Ca, Ce, Cr, Cs, Cu, K, Mg, $\mathrm{Na}, \mathrm{Ni}, \mathrm{Pb}, \mathrm{Si}, \mathrm{Zn}$. 


\section{DATA REDUCTION, VALIDATION, AND VERIFICATION}

Test data will be collected during this program to document and interpret the melter operation and meet the objectives. Data gathered during this program will include

- Melter system process data, including recorded DAS output and hand-recorded data sheets

- Records and results of all collected samples

- Offgas sampling and analysis data from the stack sampling subcontractor, including CEMS data and manual measurements and sampling data.

As much data as possible will be reduced and validated at the time of the field test, while the test team is still onsite. The critical advantage to this approach is that if deficiencies are found, then they may be more easily remedied while still onsite. Data reduction performed onsite will include

- Global mass balances of feed material and additives, slag, metal, APCS solids, and furnace inventory

- Offgas velocity, flowrate, and gas compositions

- Particulate and metals isokinetic sampling rates

- Melter process parameters, such as averages and ranges of feedrates, power levels, and tapping rates

- Global mass balance and furnace inventory calculations, including cumulative feed input, slag and metal tapping, and APCS solids collection

- Calculate isokinetic ratios for sampling trains.

Much of the data, however, cannot be generated or validated until well after completion of the field test program. This includes essentially all of the sample analytical results. Results of the offgas particulate and metals samples and process samples will be compiled, reduced, and evaluated promptly following completion of the laboratory analysis, while the samples are still available for reanalysis if needed. Data validation and verification steps will include checking the calculations, data entries, results, and flagging questionable data.

\subsection{Data Reduction Scheme}

Data will be recorded manually on data sheets and electronically in computer files and on stripcharts and other types of recorders. Data will be reduced and analyzed using hand-held calculator programs, computer spreadsheets, and other computer programs.

Data reduction for the offgas sampling and analysis will be conducted according to the reference test procedures (see Section 3). Data reduction for the process data will be conducted according to 
correct engineering principles and using verified calculations. Data reduction activities performed following the receipt of sample analysis results includes

- Entry of analytical results in spreadsheets for the manual sample trains for calculating particulate and metals concentrations and emission rates

- Entry of analytical results in spreadsheets for the process samples for calculating total instantaneous and average compositions and toxicity characteristic leachability procedure (TCLP) results

- Mass and energy balance closure spreadsheet calculations

- Volatile metals, particulate, and TRU surrogate partitioning determinations

- Comparison and interpretation of partitioning results and properties of the slag and metal melt phases to melter operating conditions and offgas conditions.

\subsection{Data Validation}

The test data will be validated, where possible, while the test team is still onsite so that corrective actions can be made more readily if necessary. Data collected on manually completed data sheets will be reviewed by the LITCO test director. Data that will be used in calculations will be entered into the appropriate spreadsheets, while informational data will be archived in test data notebooks. These notebooks are 3-ring binder compilations of all raw and reduced data. The LITCO Test Director or designee will control these notebooks. Stripchart recorder output and hard copies of electronic data files will be similarly reviewed by the data collectors and LITCO test director for completeness and accuracy. Applicable stripchart and electronic data will be entered into spreadsheet calculations, while the stripcharts, electronic files, and hard copies are filed in the test data records.

Data validation for the offgas sampling and analysis will be performed according to the specifications in the test procedures referenced in Section 5 and analytical procedures in Section 7. Data validation for the process monitoring data will be performed according to applicable QA activities such as determination of accuracy, precision, and completeness. Applicable QA activities include comparison of redundant or similar measurements, duplicate measurements for the same process instrument on different test days, calibrations, and calculations. Data validation for the process sample analytical results will be performed according to the analytical procedures referenced in Section 7.

The CEMS data will be recorded in 1 to 2 -minute intervals. This data will be read into spreadsheets for data reduction and analysis. The following analysis will be performed using these spreadsheets:

- Identification of periods of invalid data

- Identification of calibration periods and zero, mid-span, and high-span values 
- Identification of bias checks and values

- Identification of interference checks and values

- Bias, linearity, interference, zero drift, and span drift calculations

- Flagging of bias, linearity, interference, or drift calculations that exceed limits

- Calculation of 1 or 2 -minute values corrected for interference and drift

- Calculation of average values during the entire test period and during each of the runs

- Calculation of maxima, minima, and standard deviations for the test period.

The linearity and bias calculations will be done according to procedures described in Section 5 .

\subsection{Data Validation Responsibilities}

Data validation will be the ultimate responsibility of the test directors but can be performed by a qualified designee. Before submittal of the test data to the test director or designee, however, each individual data collector will be responsible for validating the collected data based on available criteria such as completeness, comparability to other previous measurements, process conditions, and conditions of the analytical equipment. For example, the offgas sampling data will first be validated by the sample train operator based on judgement of sample representativeness; cleanliness and maintenance of sampling equipment; adherence to approved, written sample collection procedures; and completeness of field data sheets. Further validation will be based on (a) process conditions during sampling or testing, (b) consistency with expected other results, and (c) adherence to prescribed QC procedures and control limits.

\subsection{Acceptance Criteria for Test Data}

Data validation and verification will be conducted, where possible, by comparing the results of QA and data validation activities to selected acceptance criteria and control limits. Acceptance criteria specified in the test and analytical procedures referenced in Sections 5 and 7 will be used. Tables 8-1 through 8-3 summarize the most important acceptance criteria for the offgas monitoring sampling activities. The acceptance criteria for the laboratory analyses are specified in the laboratory procedures referenced in Section 5. Data quality objectives for test data are discussed in Section 9. 
Table 8-1. Offgas equipment calibrations.

\begin{tabular}{|c|c|c|c|}
\hline Apparatus & Acceptance limits & Calibration frequency & Corrective action \\
\hline Sampling nozzle & $\begin{array}{l}\text { Maximum dev. } \leq 0.002 \text { in. from } \\
\text { average diameter }\end{array}$ & Before test & $\begin{array}{l}\text { Return to glass blower for better } \\
\text { rounding/grinding }\end{array}$ \\
\hline Thermocouples (TC) & $\begin{array}{l}\text { lmpinger TC: } \pm 2^{\circ} \mathrm{F} \\
\text { Gas meter TC: } \pm 5.4^{\circ} \mathrm{F} \\
\text { Stack TC: } \pm 1.5 \% \mathrm{Abs}\end{array}$ & $\begin{array}{l}\text { Calibrate each against mercury in- } \\
\text { glass thermometer at a single point } \\
\text { near operating range; for stack TCs, } \\
\text { calibrate at } 3 \text { points }\end{array}$ & $\begin{array}{l}\text { Repair to meet specifications or do } \\
\text { not use, or plot curves and correct } \\
\text { for ranges outside acceptable limits }\end{array}$ \\
\hline Dry gas meters & $0.98 \leq Y_{m, i} / Y_{m, f} \leq 1.05$ & $\begin{array}{l}\text { Calibrate initially and when post- } \\
\text { test check } Y_{m} \text { exceeds } Y_{m} \pm 0.05\end{array}$ & $\begin{array}{l}\text { Adjust, determine a correction } \\
\text { factor, or replace }\end{array}$ \\
\hline $\begin{array}{l}\text { CEMS calibration } \\
\text { drift }\end{array}$ & $\begin{array}{l}\mathrm{SO}_{2}, \mathrm{NO}_{\mathrm{x}}: \pm 2.5 \% \text { full scale (FS) } \\
\mathrm{O}_{2}, \mathrm{CO}_{2}: \pm 0.5 \% \\
\mathrm{CO}: \pm 5 \% \text { of FS for } \\
6 \text { out of } 7 \text { days } \\
\text { Others: } \pm 10 \% \text { of FS }\end{array}$ & At least daily & $\begin{array}{l}\text { Calibrate more frequently or make } \\
\text { instrument adjustments }\end{array}$ \\
\hline Barometer & $\begin{array}{l} \pm 0.1 \text {-in. } \mathrm{Hg} \text { of } \\
\text { mercury-in-glass } \\
\text { barometer }\end{array}$ & Before and after each field test & Adjust or make correction chart \\
\hline Installed process TCs & $\pm 1.5 \%$ Abs. & $\begin{array}{l}\text { Before and after each test campaign, } \\
\text { and whenever results appear } \\
\text { questionable }\end{array}$ & $\begin{array}{l}\text { Perform maintenance on } \mathrm{TC} \text { and } \\
\text { connecting wire, or replace TC }\end{array}$ \\
\hline $\begin{array}{l}\text { Installed pressure } \\
\text { indicators and } \\
\text { transducers }\end{array}$ & $\pm 5 \%$ Abs. & $\begin{array}{l}\text { Before and after each test campaign, } \\
\text { and whenever results appear } \\
\text { questionable }\end{array}$ & $\begin{array}{l}\text { Perform maintenance, adjust, } \\
\text { determine correction factor, or } \\
\text { replace }\end{array}$ \\
\hline Installed pitots & $\begin{array}{l}\text { Meets dimensional requirements in } \\
40 \text { CFR } 60 \text { App. A, Method } 2\end{array}$ & $\begin{array}{l}\text { Before and after each test campaign, } \\
\text { and whenever results appear } \\
\text { questionable }\end{array}$ & Inspect, clean, reshape, or replace \\
\hline
\end{tabular}


Table 8-2. Summary of acceptance criteria for offgas sampling.

\begin{tabular}{|c|c|c|}
\hline Criteria & Control limits & Corrective action \\
\hline Flow alignment & Average resultant angle within 20 degrees & $\begin{array}{l}\text { Select different sample location or use EPA } \\
\text { procedures for testing under cyclonic flow conditions }\end{array}$ \\
\hline Isokinetic ratio & $100 \pm 10 \%$ & Qualify data \\
\hline Final leak rate & $\begin{array}{l}\leq 0.02 \text { acfm or } 4 \% \text { or sampling rate, whichever } \\
\text { is less }\end{array}$ & Qualify data \\
\hline Dry gas meter calibration & $\begin{array}{l}\text { Post-average factor }\left(Y_{m}\right) \text { agree } \pm 5 \% \text { of } \\
\text { pretest factor }\end{array}$ & $\begin{array}{l}\text { Adjust sample volumes using the } Y_{m} \text { that gives } \\
\text { smallest volume }\end{array}$ \\
\hline Average correction factor & $1.00 \pm 10 \%$ & Adjust the dry gas meter and recalibrate \\
\hline $\begin{array}{l}\text { Intermediate dry gas } \\
\text { meter }\end{array}$ & Calibrated annually against EPA standard & - \\
\hline $\begin{array}{l}\text { Analytical balance (top } \\
\text { loader) }\end{array}$ & $\begin{array}{l} \pm 0.1 \mathrm{~g}, \text { National Institute of Standards and } \\
\text { Technology Class F weights }\end{array}$ & Repair balance and recalibrate \\
\hline Barometric pressure & Within $2.5-\mathrm{mm} \mathrm{Hg}$ of a mercury-in-glass & Recalibrate \\
\hline
\end{tabular}


Table 8-3. Acceptance criteria for offgas continuous monitoring equipment.

\begin{tabular}{|c|c|c|}
\hline Criteria & Control limits & Corrective action \\
\hline Concentration-stratification & $\pm 10 \%$ of mean & Select new sampling site \\
\hline $\begin{array}{l}\text { Zero and span drift }\left(\mathrm{O}_{2}, \mathrm{CO}_{2}, \mathrm{CO}, \mathrm{SO}_{2}\right. \\
\left.\text { and } \mathrm{NO}_{x}\right)\end{array}$ & $\pm 3 \%$ of full scale & Apply correction to data \\
\hline Zero and span drift & $\begin{array}{l} \pm 5 \% \text { of full scale or } 10 \mathrm{ppm} \text {, whichever is } \\
\text { larger }\end{array}$ & Apply correction to data \\
\hline Leak rate & $s 4 \%$ of normal flow at operating vacuum & Locate and repair leak \\
\hline $\mathrm{NO}_{2}$ converter test & $\leq 2 \%$ conversion & Clean or replace converter \\
\hline Linearity $\left(\mathrm{O}_{2}, \mathrm{CO}_{2}, \mathrm{SO}_{2}, \mathrm{NO}_{\mathrm{x}}\right)$ & $\pm 2 \%$ of full scale & $\begin{array}{l}\text { Correct data and linearize } \\
\text { analyzer }\end{array}$ \\
\hline $\operatorname{Bias}\left(\mathrm{O}_{2}\right)$ & $\leq 0.5 \% \mathrm{O}_{2}$ response to zero gas & Locate and repair leak \\
\hline $\operatorname{Bias}\left(\mathrm{SO}_{2}, \mathrm{NO}_{\mathrm{x}}\right)$ & $\pm 5.0 \%$ response to zero gas & Correct data \\
\hline $\mathrm{CO}_{2}$ interference on $\mathrm{CO}$ & None & Correct data \\
\hline
\end{tabular}




\section{QUALITY ASSURANCE}

The QA plan for this program will include activities to (a) determine the quality of test results and (b) ensure a certain level of data quality based on data quality objectives. The level of data quality for many of the measurements and activities of this program will depend on yet-to-beestablished performance of the melter facility, onsite sample collection and analysis equipment and procedures, and laboratory analytical procedures.

\subsection{Quality Level}

This type of test program is similar to an LITCO Quality Level C program. Level C programs are those that provide "items or services whose failure would not result in any significant health or safety risk to personnel, the loss or impairment of data generation, or inadequate quality of deliverable products or data; and the applicable codes, standards, or specifications do not require any acceptance verifications of other quality assuring activities."14 Operation of the melter facility represents a significant health or safety risk to personnel in the event of some types of failure, but the operation will be performed by USBM operating personnel using their own safety, operating, and quality procedures to ensure operations safety as referenced in Section 12.

While unplanned failure of the melter operation and/or sampling, analytical, and monitoring procedures and equipment during the tests may cause the loss or impairment of some intended data generation, data meeting test objectives will still be generated. This is an applications evaluation, not a process control study or a regulatory trial production test. For example, accurate and valid particulate and metals sample analysis depends on stable operation of the melter and offgas and entrained fume flow conditions within specified parameters. Successful melter operation within the specified parameters has not yet been established for the various feed conditions. Therefore, the particulate and metals sample collection and analysis methods will be applied as rigorously as possible within the expediency and process limitations of the test conditions.

Testing will be performed to meet estimated data quality objectives based on the test methodologies. When the data quality objectives are not met, then the data quality may be impaired. However, the results will still meet the objectives of the test program as baseline data to interpret the melter performance and diagnostically evaluate the current melter design and operation.

The EPA's analogous quality level would be either Category III or IV. Category III projects are those "producing results for the purpose of evaluating and selecting basic options, or performing feasibility studies or preliminary assessments of unexplored areas that might lead to further work."15 Category IV projects are those "producing results for the purpose of assessing suppositions. ${ }^{\text {"12 }}$

The primary feature of the Phase 1 test program is to research and develop innovative applications of melter technologies and obtain as much initial and baseline data as possible for planning subsequent, more detailed tests in Phases 2 and 3. Phase 1 is not being used for final evaluation of the technology applications, nor is it being used for regulatory development or compliance purposes. For these reasons, the LITCO Quality Level C (EPA Category III-IV) is appropriate for this test program. 
The extensiveness of QC procedures is defined by the quality level assigned to the program and by the specifications in various test methodologies used in the test program. An EPA Category III project plan should include sections that describe the project, QA objectives, sampling and analysis procedures, data reduction, validation and reporting, and QC activities. ${ }^{16}$ This project is described in Section 3. The sampling and analysis procedures are described in Sections 5 and 7. Data reduction, validation, and reporting are described in Sections 6 and 8. The applicable data quality objectives and QA activities are discussed below and in Sections 9.3 and 9.4 .

\subsubsection{Data Quality Objectives}

Data quality objectives are either qualitative or quantitative statements defining the quality of data needed to support the program goals. The objectives are expressed in terms of accuracy, precision, and completeness. These terms are defined below:

- Accuracy is the difference in an average of measurements of a parameter, $X$, with an accepted reference or true value, $T$. It is usually expressed as the difference between the two values, $\mathrm{X}-\mathrm{T}$, or the difference as a percentage, $100(\mathrm{X}-\mathrm{T}) / \mathrm{T}$. It is also sometimes expressed as a ratio, $\mathrm{X} / \mathrm{T}$. Accuracy is a measure of the bias in a method.

- Precision is a mutual agreement among individual measurements of the same property, usually under prescribed similar conditions. Precision is best expressed in terms of standard deviations. Various measures of precision exist depending upon the "prescribed similar conditions."

- Completeness is the amount of valid data obtained from a measurement system compared to the amount that was expected to be obtained under correct normal conditions. Completeness is usually calculated as the number of valid measurements divided by the number of required or planned measurements times $100 \%$.

The data quality objectives for the critical measurements in this test program are shown in Table 9-1. These data quality objectives will be used to support decisions concerning test validity and adequacy with respect to the program goals. Precision and accuracy for EPA reference method procedures are as prescribed by the EPA in $40 \mathrm{CFR} 60$ Appendix $A$ as those required for a decision to be made regarding emissions compliance. Those indicated as EPA draft and other procedures have data quality objectives assigned at levels within the capabilities of the method, which may not yet be sufficiently documented.

The completeness levels are the minimum percentage typically achievable for sampling and analysis of this type. While it is not expected that any samples will be invalidated because of operator error, events such as power failures, failed connections, and broken glassware occasionally occur. For this reason, the completeness goals are not set at $100 \%$. The completeness values also do not include the possibility of invalid measurements from failure of the pilot scale arc melter. Since this melter program is a research and development program rather than a routinely run process, the potential of melter operational failures during any given sample period is significant. Melter system operational failures will not affect the completeness of the various measurements. 
Table 9-1. Data quality objectives for critical measurements.

\begin{tabular}{|c|c|c|c|c|}
\hline Measurement & Reference or method & $\begin{array}{l}\text { Precision } \\
\text { (\%) }\end{array}$ & $\begin{array}{l}\text { Accuracy } \\
(\%)\end{array}$ & $\begin{array}{c}\text { Completeness } \\
\text { (\%) }\end{array}$ \\
\hline Feed additive weights, product weights & Magna-View Floor Scale (Howe Richardson Co.) & $\pm 1 \mathrm{lb}$ & \pm 1 & 90 \\
\hline Process temperatures & Thermocouples & ND & $\pm 1.5 \%$ & 90 \\
\hline Static pressures & Pressure transducers & ND & \pm 10 & 90 \\
\hline Feedrate & Calibrated feed screws & \pm 5 & \pm 5 & 90 \\
\hline Gaseous flowrates & Pitot tubes, other flowmeters & ND & \pm 10 & 90 \\
\hline Scrubber liquor rate & TBD & TBD & TBD & 90 \\
\hline Scrubber blowdown rate & TBD & TBD & TBD & TBD \\
\hline EPA 29 metals & EPA Draft Method 29 & \pm 50 & $\pm 50^{\mathrm{a}}$ & 80 \\
\hline EPA 29 total particulate & EPA Method 5 (from EPA Draft Method 29 Train) & \pm 20 & $\pm 20^{a}$ & 80 \\
\hline $\mathrm{O}_{2}, \mathrm{CO}_{2}$ & EPA Method 3A & \pm 10 & \pm 10 & 80 \\
\hline $\mathrm{SO}_{2}$ & EPA Method 6C & \pm 10 & \pm 10 & 80 \\
\hline $\mathrm{NO}_{\mathrm{x}}$ & EPA Method 7E & \pm 10 & \pm 10 & 80 \\
\hline $\mathrm{CO}$ & EPA Method 10 & \pm 10 & \pm 10 & 80 \\
\hline Stack gas moisture from EPA 29 train & EPA Method 5 (from EPA Draft Method 29 Train) & \pm 20 & ND $^{\mathbf{b}}$ & 80 \\
\hline Stack gas velocity and flowrate from EPA 29 train & EPA Method 2 (from EPA Draft Method 29 Train) & ND & ND & 80 \\
\hline Stack gas temperature from EPA 29 train & EPA Method 2 (from EPA Draft Method 29 Train) & ND & $\pm 3^{c}$ & 80 \\
\hline Process samples, full set metals ${ }^{a, d}$ & SW846 6010 or 7000 series, USBM procedures & $<20$ & \pm 20 & 90 \\
\hline Process samples, $\mathbf{P}$ & AOAC method total phosphorous determination ${ }^{e}$ & TBD & TBD & 80 \\
\hline Process samples, $\mathbf{S}$ & LECO CS 144 gas analyzer & TBD & TBD & 80 \\
\hline Process samples, total C & LECO CS 144 & $<20$ & \pm 20 & 90 \\
\hline Process samples, $\mathrm{Cl}$ & USBM ALRC CI Analysis & $<20$ & \pm 20 & 90 \\
\hline \multicolumn{5}{|l|}{ TBD $=$ To Be Determined. } \\
\hline \multicolumn{5}{|l|}{ a. Analytical phase onty. } \\
\hline \multicolumn{5}{|l|}{ b. Not determinable within limits of method precision. } \\
\hline \multicolumn{5}{|l|}{ c. Relative accuracy compared to thermometer. } \\
\hline \multicolumn{5}{|c|}{ d. Full set metals (18 metals)-Al, As, Ba, Ca, Ce, Cr, Cs, Cu, Fe, K, Mg, Na, Ni, Pb, Si, Ti, Zn, and Zr. } \\
\hline \multicolumn{5}{|c|}{ e. Official Methods of malysis of the Official Agricultural Chemists, 10th Edition, 1965, pages 11-13. } \\
\hline
\end{tabular}


In the event that the specified completeness levels are not attained, corrective action will be taken immediately. However, much of the data validation will not be complete until after the testing phase has ended. In this case, it may not be possible to take corrective action to meet the data quality objectives (e.g., if the analytical laboratory irrecoverably contaminates or loses a sample).

\subsection{Quality Control Methods}

QC methods specified by the various testing, analytical, and routine procedures will be used to determine the quality of the test data and ensure that certain minimum quality objectives are achieved. QC activities will include data reduction, validation, and verification procedures as summarized in Section 8. QC activities will also include these features:

- Equipment maintenance and calibration procedures and schedules, controlled by the USBM and subcontractor personnel

- Standard calculation routines using hand-held calculators and computer spreadsheets, prepared and controlled by the USBM, LITCO or subcontractor personnel, depending on the specific program or spreadsheet

- Sample blanks, spikes, duplicates, and other analytical QC procedures as specified in the analytical procedures

- Organization and documentation of all calibration records, run sheets, data sheets, process logs, CEMS computer files and hard-copy data, calculation sheets, and spreadsheet files and printouts

- Internal QA audits and checks (see Section 9.4).

Equipment maintenance and calibrations are summarized in Sections 8, 10.3, and 10.4. Internal QA audits and checks are summarized in Section 9.4.

General QC checks that will be used during field testing and laboratory analysis are summarized in Section 8.4 and in Table 9-2. There are also a number of specific QC checks required for the specific test procedures. QC activities performed for manual sampling methods include

- Filter must be maintained at $250 \pm 25^{\circ} \mathrm{F}$. The probes and sample lines must be maintained at a temperature that prevents moisture condensation.

- $\quad$ Reading of the dry gas meter, $\Delta P, \Delta H$, temperature, and vacuum pump must be made during sampling at each traverse point.

- All sampling data forms will be reviewed daily. Incomplete or inaccurate data will not be acceptable.

- Only precleaned containers will be used to sample recovery. 
Table 9-2. Internal QC checks.

\begin{tabular}{|c|c|c|}
\hline Item & QC check & Frequency \\
\hline Equipment & $\begin{array}{l}\text { Inspect glassware for cracks; filters for tears, } \\
\text { appearance; fittings for leaks; pitot tubes; } \\
\text { probes for cleanliness; sample lines for } \\
\text { cleanliness, leaks; general equipment set-up for } \\
\text { ease of operation }\end{array}$ & Daily \\
\hline Operation & Review specific procedure checklist items & Daily spot checks \\
\hline $\begin{array}{l}\text { Data } \\
\text { records }\end{array}$ & $\begin{array}{l}\text { Review calibration sheets, run sheets, operator } \\
\text { logbooks, analytical sheets, tracking sheets, } \\
\text { custody sheets for accuracy, completeness }\end{array}$ & Daily \\
\hline Test results & $\begin{array}{l}\text { Inspect for obvious errors, trends, } \\
\text { completeness, precision, accuracy }\end{array}$ & Daily \\
\hline $\begin{array}{l}\text { Sample } \\
\text { handling }\end{array}$ & $\begin{array}{l}\text { Inspect sample containers before and after use } \\
\text { for cleanliness, application; inspect sample } \\
\text { labels for accuracy, completeness }\end{array}$ & Daily \\
\hline $\begin{array}{l}\text { Analytical } \\
\text { results }\end{array}$ & $\begin{array}{l}\text { Review for obvious errors, trends, blanks, } \\
\text { spikes, recovery, completion of requested } \\
\text { work, timeliness }\end{array}$ & $\begin{array}{l}\text { Every receipt of } \\
\text { analytical results }\end{array}$ \\
\hline
\end{tabular}

QC activities specific to particulate and metals sampling include

- Recording weights of filter from field blanks before and after train assembly to determine if filter tare weight is changed by handling.

- Calibration of analytical balance before and after particulate weights are determined.

- Visual inspection of system equipment, daily

- Monitoring of key temperatures, such as instrument, chiller, and line temperatures, every test

- Monitoring of sample flowrates, every test

- Interference checks to determine effects of one gas species on the detection of another species at least once at each site.

Internal control checks for the laboratory analysis methods include blanks, spikes, duplicates, and split samples as specified in the analytical procedures. A summary of the laboratory QC checks for the metals and particulate sampling is shown in Table 9-3. Where possible, the specified laboratory QC checks will be followed rigorously. Deviations from the specified QC checks will be made only when the deviations do not impair the usability of the test results for this test program. 
Table 9-3. Internal QC sample matrix for particulate and metals measurements for the MMT samples and process samples.

\begin{tabular}{|c|c|c|c|}
\hline $\begin{array}{l}\text { QC sample } \\
\text { type }\end{array}$ & Description & Analyze & Archive \\
\hline $\begin{array}{l}\text { Reagent } \\
\text { blanks }^{\mathbf{a}}\end{array}$ & $\begin{array}{l}\text { Filters } \\
5 \% \mathrm{HNO}_{3} / 10 \% \mathrm{H}_{2} \mathrm{O}_{2} \\
\text { Deionized water }\end{array}$ & $\begin{array}{l}\text { Archive } \\
\text { Archive } \\
\text { Archive }\end{array}$ & 1 \\
\hline $\begin{array}{l}\text { Recovery } \\
\text { blanks }{ }^{\mathrm{b}}\end{array}$ & $\begin{array}{l}\text { Acetone } \\
0.1 \mathrm{~N} \mathrm{HNO}_{3}\end{array}$ & $\begin{array}{l}\text { Archive } \\
\text { Archive }\end{array}$ & 1 \\
\hline Proof blanks & Complete train & Archive & 1 \\
\hline Method QC & $\begin{array}{l}\text { Per procedure except } \\
\text { as modified in this } \\
\text { test plan }\end{array}$ & & \\
\hline $\begin{array}{l}\text { Spike } \\
\text { sample }^{c}\end{array}$ & Digested sample & None & \\
\hline Split $^{d}$ & Digested sample & $\begin{array}{l}1 \text { of each sample } \\
\text { type, (slag, metal, } \\
\text { and each APCS } \\
\text { sample type, and } \\
\text { MMT sample) }\end{array}$ & \\
\hline $\begin{array}{l}\text { Duplicate } \\
\text { analysis }^{\mathbf{e}}\end{array}$ & Digested sample & $\begin{array}{l}1 \text { of each sample } \\
\text { type }\end{array}$ & \\
\hline \multirow{2}{*}{\multicolumn{4}{|c|}{$\begin{array}{l}\text { a. Reagent blends are samples of unused raw reagents used in the sample collection process. } \\
\text { b. Recovery blanks are samples of unused solutions used for sample recovery. } \\
\text { c. Spike sample is the addition of a known amount of a standard to sample to evaluate matrix effects } \\
\text { and other potential analytical artifacts on the analytical results. Spikes will be done only for a few yet- } \\
\text { to-be determined analytes. }\end{array}$}} \\
\hline & & & \\
\hline \multicolumn{4}{|c|}{ d. Split samples are those that are split into two or more portions, and each portion is analyzed. } \\
\hline e. Duplicate a & repeat analysis on a separ & of the original samp & \\
\hline
\end{tabular}


Such deviations will be made with the prior approval of the USBM Project Manager and LITCO Principal Investigator.

All pertinent offgas sampling records in the field will be documented and filed to ensure against loss of key data. Key records include

- Calibrations

- Maintenance records

- Manual method run sheets and weigh sheets.

Internal QA/QC checks for CEM testing include:

- Calibrations, every test

- Leak checks, every test

- Linearity checks, every 3 days or more frequently

- Bias checks, every 3 days or more frequently

- CEMS stripcharts and calibration sheets

- Process data sheets

- Hand calculator calculation forms

- Manual method computer spreadsheets, hard copies

- CEMS computer spreadsheets, hard copies

- Other computer spreadsheets, hard copies

- Floppy disks, manual method spreadsheets, CEMS data disks and spreadsheets, and process data

- QA audit sheets.

\subsection{Routine Procedures}

Standard operating procedures (SOPs) developed by the USBM test team or test and analytical procedures promulgated by the EPA will be observed where possible during the test program. QC activities relevant to these procedures are summarized in Section 9.2. 


\subsection{Internal Audit System}

Internal QA audits and checks to ensure satisfactory completion of these activities will be conducted by the LITCO test director or designee. These audit activities include audit checklists, performance audits, control charts to visually show trends of key test results, and standard data sets to verify the accuracy of calculated results. The internal audits planned for this program are summarized in Table 9-4.

Table 9-4. Internal audits and QA checks.

\begin{tabular}{lll}
\hline Type of audit & \multicolumn{1}{c}{ Audit subject } & \multicolumn{1}{c}{ When conducted } \\
\hline Systems & $\begin{array}{l}\text { Data records-CEMS, manual sampling, process } \\
\text { data collection }\end{array}$ & End of each test day \\
Performance & $\begin{array}{l}\text { CEMS and manual method equipment field } \\
\text { calibrations }\end{array}$ & $\begin{array}{l}\text { Per EPA reference } \\
\text { methodology }\end{array}$ \\
Control charts & $\begin{array}{l}\text { Key results and calculations (key process } \\
\text { temperatures, flowrates, pressures, \% I, \% } \\
\text { moisture, velocity, flowrates } \mathrm{O}_{2}, \mathrm{CO}_{2}, \text { etc.) and } \\
\text { for CEMS drift. }\end{array}$ & End of each test day \\
Control charts & $\begin{array}{l}\text { Laboratory surrogate recoveries spikes, blanks, } \\
\text { duplicates }\end{array}$ & $\begin{array}{l}\text { During and/or after sample } \\
\text { analysis }\end{array}$ \\
Performance & Hand calculator and computer spreadsheet & $\begin{array}{l}\text { Once before use and after } \\
\text { each modification }\end{array}$ \\
\hline
\end{tabular}




\section{EQUIPMENT AND INSTRUMENTS}

A variety of process equipment, test equipment, and process monitoring, sampling, and analytical instrumentation will be used during this test program. The melter process equipment and instrumentation is in place at the USBM ALRC. The main features and components are summarized in Section 3.

The sampling and analytical equipment is specified in the appropriate test procedures. The test procedures and key equipment items are summarized in Section 5. The analytical laboratory instrumentation is also specified in the applicable procedures, which are summarized in Section 7.

\subsection{Range, Accuracy, and Tolerance Levels}

The range, accuracy, and tolerance levels of the process monitoring and sampling equipment is subject to the design and operation of the melter facility. These specifications were designed to be appropriate for safe and effective operation of the melter and allow sufficient tolerance of the instrumentation to the high temperatures of the process. Specific instrumentation that can be easily calibrated such as pitot tubes for the offgas flowrate monitoring will be calibrated. Other instrumentation is not readily calibrated. Instruments of this type will maintained and used according to manufacturer's specifications to ensure accuracy and reliability of the results.

The range, accuracy, and tolerance levels of the offgas monitoring and sampling equipment and analytical equipment are specified in the test methodologies. To the extent possible, these performance levels will be strictly complied with during the test program. The test methodologies are referenced in Sections 5 and 7.

\subsection{Calibration and Standardization Procedures}

Calibrations will be performed according to the specified procedures discussed in Sections 5, 7, and 8 for field measurements, laboratory analyses, and QA activities. Some deviations may be necessary from the specified laboratory instrument calibrations, because reliable calibration standards for some analytes may not be available.

\subsection{Preventive Maintenance}

Preventive maintenance is performed for the melter system to ensure proper and safe operation of all components. Preventive maintenance for the sampling and analytical equipment is done to help ensure accurate and reliable measurements and sample collection. Before testing, all melter system components, instrumentation, and sample equipment will be inspected and checked for proper operation. Preventive maintenance for the melter system and test equipment will continue during the demonstration tests. The most critical equipment that will be inspected and maintained on a daily basis include

- Melter system: Feed motors, augers, hoppers, feed tubes, furnace roof, refractory, tapping fixture, and APCS ducting. 
- Instrumentation: Thermocouples, pressure transducers, flowmeters, sample pumps and lines, pitot tubes, sample conditioning equipment, and CEMS instrumentation. 


\section{SUPPLIES, UTILITIES, AND FACILITIES}

The expected necessary facilities and utilities are already in place at the ALRC. Supplies are also available at the ALRC, but specific reagents and materials needed for the test series will be maintained on-hand at the melter facility. 


\section{HEALTH AND SAFETY}

The USBM ALRC has an operations safety plan administered through the following documents:

1. Chemical Hygiene Plan, January 22, 1991

2. Procedures for Startup and Resumed Activities, October 20, 1992

3. Job Safety Analysis for Operation of Three-Phase Electric Arc Melting Furnace, May 4, 1993

4. Standard Operating Procedure, Waste Calcining Facility Arc Melting Furnace, May 4, 1993.

These documents also entail SOPs for operating the melter and ancillary equipment.

The following potentially hazardous conditions are possible during the melting tests:

- Hot surfaces and molten slags and metals

- Large volumes of hot flammable gases (such as $\mathrm{CO}, \mathrm{H}_{2}, \mathrm{CH}_{4}$ ) and poisonous gases $(\mathrm{CO}$, $\mathrm{HCl}, \mathrm{NO}_{\mathrm{x}}$ ) will be produced within the furnace

- Fugitive emissions from various locations in the melter system.

Reasonable steps that will be taken to ensure safe operation, including

- Roof fan will be replaced with three silent models that will change the air in the building every 2 minutes.

- Continuous monitors for $\mathrm{HCl}$ and $\mathrm{NO}_{x}$ will be used to indicate and warn of furnace emissions.

- Appropriate personnel protective equipment (PPE) will be required for all test personnel. Personal air supply systems also will be required when the monitors indicate that respirators are insufficient for personnel protection. All personnel attending the furnace shall wear protective clothing. gloves, boots, and hard hats. Heat resistant suits are required for the tapping $\mathrm{crcw}$.

- Control room will be provided with a positive air supply to protect the furnace operator and support personnel.

- Mandatory safety review and discussion of test procedures will be conducted before the test series for all test team personnel. 


\section{RESIDUALS MANAGEMENT}

Residuals from this test program will include the (a) slag, metal, and APCS solids, (b) a large number of samples, (c) possibly some spent baghouse bags, and (c) unused feed materials. The responsibility for handling and disposal of these residuals presently resides with both USBM and with LITCO.

Any untreated, unprocessed surrogate waste will be stored at the ALRC for use in additional waste feed characterization studies and in the planned Phase 3 tests. The secondary streams residuals are expected to be nonhazardous, and TCLP analyses will be performed to verify this assumption. Portions of the secondary stream materials will be archived for use in any additional studies, while the remainder will be disposed of at the local landfill. Any nonhazardous samples that are no longer needed in the archive will also be disposed of in the local landfill.

Some of the APCS solids may be hazardous. These will be disposed of (when no longer needed in archive) using an approved, certified, hazardous waste disposal company. 


\section{REFERENCES}

1. S. O. Bates, Definition and Compositions of Standard Wastestreams for Evaluation of Buried Waste Integrated Demonstration Treatment Technologies, EGG-WTD-10660, EG\&G Idaho, Inc., June 1993.

2. J. L Mayberry, W. J. Quapp, F. Feizollahi, J. C. Del Signore, and J. McFee, Preliminary Systems Design Study Assessment Report, EGG-WTD-9594, EG\&G Idaho, Inc., June 1991.

3. A. D. Donaldson, R. J. Carpenedo, and G. L. Anderson, Melter Development Needs Assessment for RWMC Buried Wastes, EGG-WTD-9911, EG\&G Idaho, Inc., February 1992.

4. T. L. Eddy, P. C. Kong, B. D. Raivo, and G. L. Anderson, Thermal Processing Systems Concepts and Considerations for RWMC Buried Wastes, EGG-WTD-10058, EG\&G Idaho, Inc., February 1992.

5. P. C. Kong, G. A. Reimann, S. O. Bates, B. A. Detering, and G. L. Anderson, Final Waste Form Option Scoping Studies for RWMC Wastes, EGG-WTD-10363, Rev 0, EG\&G Idaho, Inc., March 1993.

6. J. K. Cotchen and H. F. Davis, "Electric Arc Furnace Processing of Solid Wastes," International Symposium, Process of Residues and Effluents, TMS Annual Meeting, 1992.

7. L. C. Rounds and J. K. McDonald, Buried Waste Integrated Demonstration Technology Test Plan Guidance, EGG-WTD-9800, Rev. 1, EG\&G Idaho, Inc., January 1994.

8. L. L. Oden, W. K. O'Conner, J. S. Hansen, and J. C. White, Vitrification of Municipal Refuse Combustor Residues by Electric Arc Furnace Melting, U.S. Bureau of Mines, Albany Research Center, March 1993.

9. G. L. Anderson, A. D. Donaldson, and N. R. Soelberg, Test Plan for the FY-93 Phase I Baseline Tests for the BWID Arc Melter Vitrification Project, EGG-WTD-10862, Revision 0, July 1993.

10. L. L. Oden, W. K O'Connor, P. C. Tumer, N. R. Soelberg, and G. L. Anderson, Baseline Tests for Arc Melter Vitrification of INEL Buried Wastes, EGG-WTD-10981, EG\&G Idaho, Inc., November 19, 1993.

11. J. G. Sotter, J. A. Arnot, and T. M. Brown, Guidelines for Fireside Testing in Coal-Fired Power Plants, EPRI CS-5552, Project 1891-3 Final Report, March 1988.

12. N. R. Soelberg, A. G. Chambers, G. L. Anderson, L L. Oden, W. K. O'Connor, and P. C. Turner, Arc Melter Demonstration Baseline Test Results, EGG-WTD-11138, EG\&G, Idaho, Inc., July 1994.

13. EG\&G Idaho, Inc., "Engineering Standard Practices (ESP) Manual," Section No. ESP 4.4.1 Rev. A, DRR No. EG-864, March 25, 1991.

14. EG\&G Idaho Inc., Quality Manual. 
15. Environmental Protection Agency, Preparing Perfect Project Plans, EPA/600/9-89/087, EPA Risk Reduction Engineering Laboratory, October 1989.

16. Environmental Protection Agency, Quality Assurance Handbook for Air Pollution Measurements Systems, Vol II, EPA-600/4-77-027b. 


\section{Appendix A}

\section{BWID Nominal Standard Waste Mixture Tables}


A-2 
Table A-1. Additive compositions for the BWID Phase 2 feed mixtures.

\begin{tabular}{|c|c|c|c|c|c|c|c|c|c|c|c|c|c|c|c|c|c|}
\hline \multirow[b]{3}{*}{ Componert } & \multicolumn{6}{|c|}{ Composition in Weighe \% } & \multirow{3}{*}{  } & \multirow{2}{*}{\multicolumn{5}{|c|}{ Design Composition of Feed Mixtures ( $(\%)$}} & \multirow{2}{*}{\multicolumn{5}{|c|}{$\begin{array}{l}\text { Design Composition of Feed Mixtures (x) } \\
\text { Renormalived for Added Spites end Buffer }\end{array}$}} \\
\hline & \multirow{2}{*}{$\begin{array}{c}\text { Hazardous } \\
\text { Metals }\end{array}$} & \multirow{2}{*}{$\begin{array}{c}\text { Solid } \\
\text { Combustibles }\end{array}$} & \multirow{2}{*}{$\begin{array}{l}\text { Silicated } \\
\text { Organics } \\
\text { (743) }\end{array}$} & \multirow{2}{*}{$\begin{array}{c}\text { Nitrate } \\
\text { (Evaporator) } \\
\text { Salts (745) }\end{array}$} & \multirow{2}{*}{$\begin{array}{l}\text { Metal } \\
\text { Hydroxide } \\
\text { Studges } \\
(741,742) \\
\end{array}$} & \multirow{2}{*}{$\begin{array}{c}\text { Nominal } \\
\text { Composition }\end{array}$} & & & & & & & & & & & \\
\hline & & & & & & & & Nom -90 & Nom-80 & Nom-70 & Nom-60 & Nom-50 & Nom-90 & Nom- $-\infty$ & Nom- -0 & Nom 60 & Nom-so \\
\hline Ce spike & & & & & & & & & & & & & 0.50 & 0.50 & 0.50 & 0.50 & 0.50 \\
\hline Soil & & & & & & & & 90.00 & 80.00 & 70.00 & 60.00 & 50.00 & 67.72 & 59.12 & 50.98 & 43.17 & 35.60 \\
\hline Lime & & & & & & & & & & & & & 19.69 & 17.10 & 14.64 & 12.27 & 9.9 \\
\hline Mill scale & & & & & & & & & & & & & 4.51 & 8.38 & 11.82 & 14.94 & 17.82 \\
\hline & & & & & & & & & & & & & & & & & \\
\hline Carton streel & 60.00 & & & & & 22.50 & & 2.25 & 4.50 & 6.75 & 9.00 & 11.25 & 1.71 & 3.35 & 4.96 & 6.55 & 8.12 \\
\hline Stainless steecl & 30.50 & & & & & 11.44 & & 1.14 & 2.29 & 3.43 & 4.58 & 5.72 & 0.87 & 1.70 & 2.52 & 3.33 & 4.13 \\
\hline Ahminum & 5.00 & & & & & 1.88 & & 0.19 & 0.38 & 0.56 & 0.75 & 0.94 & 0.14 & 0.28 & 0.41 & 0.55 & 0.68 \\
\hline Zirconium & 2.50 & & & & & 0.94 & & 0.09 & 0.19 & 0.28 & 0.38 & 0.47 & 0.07 & 0.14 & 0.21 & 0.27 & 0.34 \\
\hline Copper & 1.50 & & & & & 0.56 & & 0.06 & 0.11 & 0.17 & 0.23 & 0.28 & 0.04 & 0.08 & 0.12 & 0.16 & 0.20 \\
\hline Lead & 0.50 & & & & & 0.19 & 37.50 & 0.02 & 0.04 & 0.06 & 0.08 & 0.09 & 0.01 & 0.03 & 0.04 & 0.05 & 0.07 \\
\hline WD Polyethylene. (-CH2-CH2-) & & 22.00 & & & & 5.50 & & 0.55 & 1.10 & 1.65 & 2.20 & 2.75 & 0.42 & 0.82 & 1.21 & 1.60 & 1.99 \\
\hline Wood pellets (paper), (CGH10OS)n & & 13.00 & & & & 3.25 & & 0.33 & 0.65 & 0.98 & 1.30 & 1.63 & 0.25 & 0.48 & 0.72 & 0.95 & 1.17 \\
\hline Wood pellests (wood), (C6H1005)n & & 46.00 & & & & 11.50 & & 1.15 & 2.30 & 3.45 & 4.60 & 5.73 & 0.87 & 1.71 & 2.54 & 3.35 & 4.15 \\
\hline Neoprene rubber, (-CH2CCl-CHCH2-)n & & 10.00 & & & & 2.50 & & 0.25 & 0.50 & 0.75 & 1.00 & 1.25 & 0.19 & 0.37 & 0.55 & 0.73 & 0.90 \\
\hline Oil Dri sonbent & & 6.00 & & & & 1.50 & & 0.15 & 0.30 & 0.45 & 0.60 & 0.75 & 0.11 & 0.22 & 0.33 & 0.44 & 0.54 \\
\hline Wood pellets (clooh), (C6H 10O5)n & & 3.00 & & & & 0.75 & 25.00 & 0.08 & 0.15 & 0.23 & 0.30 & 0.38 & 0.06 & 0.11 & 0.17 & 0.22 & 0.27 \\
\hline Texaco Regal Oil, (CH2)n & & & 18.03 & & & 4.06 & & 0.41 & 0.81 & 1.22 & 1.62 & 2.03 & 0.31 & 0.60 & 0.89 & 1.18 & 1.46 \\
\hline Hydraulic oil, (CH2)n & & & 18.67 & & & 4.20 & & 0.42 & 0.84 & 1.26 & 1.68 & 2.10 & 0.32 & 0.63 & 0.93 & 1.22 & 1.52 \\
\hline Wheel bearing grease, (CH2)n & & & 2.18 & & & 0.49 & & 0.05 & 0.10 & 0.15 & 0.20 & 0.25 & 0.04 & 0.07 & 0.11 & 0.14 & 0.18 \\
\hline PVC. (-CH3Cl-) & & & 30.32 & & & 6.82 & & 0.68 & 1.36 & 2.05 & 2.73 & 3.41 & 0.52 & 1.02 & 1.51 & 1.99 & 2.46 \\
\hline Microcel E & & & 26.67 & & & 6.00 & & 0.60 & 1.20 & 1.80 & 2.40 & 3.00 & 0.45 & 0.89 & 1.32 & 1.75 & 2.17 \\
\hline Oil Dri & & & 4.00 & & & 0.90 & & 0.09 & 0.18 & 0.27 & 0.36 & 0.45 & 0.07 & 0.13 & 0.20 & 0.26 & 0.32 \\
\hline HD Polyethylene, (-CH2-CH2-) & & & 0.18 & & & 0.04 & 22.51 & 0.00 & 0.01 & 0.01 & 0.02 & 0.02 & 0.00 & 0.01 & 0.01 & 0.01 & 0.01 \\
\hline NaNO3 & & & & 54.00 & & 2.70 & & 0.27 & 0.54 & 0.81 & 1.08 & 1.35 & 0.20 & 0.40 & 0.60 & 0.79 & 0.97 \\
\hline$\overline{\mathrm{KNO} 3}$ & & & & 27.00 & & 1.35 & & 0.14 & 0.27 & 0.41 & 0.54 & 0.68 & 0.10 & 0.20 & 0.30 & 0.39 & 0.99 \\
\hline $\mathrm{NaCl}$ & & & & 3.06 & & 0.15 & & 0.02 & 0.03 & 0.05 & 0.06 & 0.08 & 0.01 & 0.02 & 0.03 & 0.04 & 0.06 \\
\hline $\mathrm{N} 22 \mathrm{SO}_{4}$ & & & & 2.97 & & 0.15 & & 0.01 & 0.03 & 0.04 & 0.06 & 0.07 & 0.01 & 0.02 & 0.03 & 0.04 & 0.06 \\
\hline $\mathrm{NaOH}$ & & & & 2.97 & & 0.15 & & 0.01 & 0.03 & 0.04 & 0.06 & 0.07 & 0.01 & 0.02 & 0.03 & 0.04 & 0.05 \\
\hline Water & & & & 9.82 & & 0.49 & & 0.05 & 0.10 & 0.15 & 0.20 & 0.25 & 0.04 & 0.07 & 0.11 & 0.14 & 0.18 \\
\hline LD Polyethylene, (-CH2-CH2-) & & & & 0.18 & & 0.01 & 5.00 & 0.00 & 0.00 & 0.00 & 0.00 & 0.00 & 0.00 & 0.00 & 0.00 & 0.00 & 0.00 \\
\hline $\mathrm{Al}(\mathrm{OH})^{3}$ & & & & & 11.50 & 1.15 & & 0.12 & 0.23 & 0.35 & 0.46 & 0.58 & 0.09 & 0.17 & 0.25 & 0.33 & 0.42 \\
\hline $\mathrm{Fe}(\mathrm{OH}) 3$ & & & & & 10.80 & 1.08 & & 0.11 & 0.22 & 0.32 & 0.43 & 0.54 & 0.08 & 0.16 & 0.24 & 0.31 & 0.39 \\
\hline $\mathrm{C}(\mathrm{OH}) 2$ & & & & & 6.60 & 0.66 & & 0.07 & 0.13 & 0.20 & 0.26 & 0.33 & 0.05 & 0.10 & 0.15 & 0.19 & 0.24 \\
\hline $\mathrm{Mg}(\mathrm{OH}) 2$ & & & & & 7.90 & 0.79 & & 0.08 & 0.16 & 0.24 & 0.32 & 0.40 & 0.06 & 0.12 & 0.17 & 0.23 & 0.29 \\
\hline $\mathrm{KHO}$ & & & & & 7.40 & 0.74 & & 0.07 & 0.15 & 0.22 & 0.30 & 0.37 & 0.06 & 0.11 & 0.16 & 0.22 & 0.27 \\
\hline H2O & & & & & 4.02 & 4.40 & & 0.44 & 0.88 & 1.32 & 1.76 & 2.20 & 0.33 & 0.66 & 0.97 & 1.28 & 1.59 \\
\hline Portland Cement & & & & & 11.60 & 1.16 & & 0.12 & 0.23 & 0.35 & 0.46 & 0.58 & 0.09 & 0.17 & 0.26 & 0.34 & 0.42 \\
\hline LD Polyethylene, (-CH2-CH2-) & & & & & 0.18 & 0.02 & & 0.00 & 0.00 & 0.01 & 0.01 & $0.0 !$ & 0.00 & 0.00 & 0.00 & 0.01 & 0.01 \\
\hline & & & & & & & 10.00 & 0.00 & 0.00 & 0.00 & 0.00 & 0.00 & 0.00 & 0.00 & 0.00 & 0.00 & 0.00 \\
\hline Toals & 100.00 & 100.00 & 100.04 & 100.00 & 100.00 & 100.01 & 100.01 & 100.00 & 100.00 & 100.00 & 100.00 & 100.00 & 100.00 & 100.00 & 100.00 & 100.00 & 100.00 \\
\hline Added lime $(\mathrm{b} / 100 \mathrm{~b} \mathrm{~b}$ Nom- $\mathrm{xx}$ mixures) & & & & & & & & 25.98 & 22.94 & 19.90 & 16.86 & 13.81 & & & & & \\
\hline Added mill scak (b/100 bo Nom-xx mixtu & & & & & & & & 5.94339 & 11.24699 & 16.07535 & 20.53102 & 24.68387 & & & & & \\
\hline 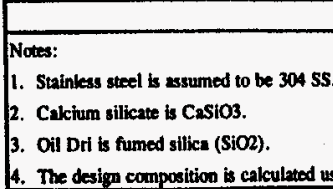 & $\operatorname{sing}$ & 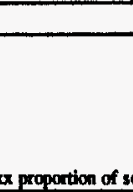 & & & & & & & & & & & & & & & \\
\hline
\end{tabular}


Table A-2. Calculated species compositions for the BWID Phase 2 feed mixtures.

\begin{tabular}{|c|c|c|c|c|c|c|c|c|c|c|c|c|c|c|}
\hline \multirow[b]{2}{*}{ Species } & \multirow[b]{2}{*}{ Lime } & \multirow{2}{*}{$\begin{array}{c}\text { Normalized } \\
\text { RWMC } \\
\text { Soil }\end{array}$} & \multirow[b]{2}{*}{$\begin{array}{l}\text { Mill } \\
\text { scale }\end{array}$} & \multirow[b]{2}{*}{$\begin{array}{c}\text { Nominal } \\
\text { Waste }\end{array}$} & \multicolumn{5}{|c|}{ Design Composition of Feed Mixtures (\%) } & \multicolumn{5}{|c|}{$\begin{array}{l}\text { Design Composition of Feed Mixtures (\%) } \\
\text { Renormalized for Added Spikes and Buffer }\end{array}$} \\
\hline & & & & & $\begin{array}{l}\text { NOM-90 } \\
\text { Feed }\end{array}$ & $\begin{array}{l}\text { NOM-80 } \\
\text { Feed }\end{array}$ & $\begin{array}{l}\text { NOM-70 } \\
\text { Feed }\end{array}$ & $\begin{array}{l}\text { NOM-60 } \\
\text { Feed }\end{array}$ & $\begin{array}{l}\text { NOM-50 } \\
\text { Feed }\end{array}$ & $\begin{array}{c}\text { NOM-90 } \\
\text { Feed }\end{array}$ & $\begin{array}{l}\text { NOM-80 } \\
\text { Feed }\end{array}$ & $\begin{array}{l}\text { NOM-70 } \\
\text { Feed }\end{array}$ & $\begin{array}{l}\text { NOM-60 } \\
\text { Feed }\end{array}$ & $\begin{array}{c}\text { NOM-50 } \\
\text { Feed }\end{array}$ \\
\hline Ce spike & & & & & & & & & & 0.50 & 0.50 & 0.50 & 0.50 & 0.50 \\
\hline Soil & & & & & 90.00 & 80.00 & 70.00 & 60.00 & 50.00 & 67.72 & 59.12 & 50.98 & 43.17 & 35.60 \\
\hline Lime & & & & & & & & & & 19.69 & 17.10 & 14.64 & 12.27 & 9.97 \\
\hline Mill scale & & & & & & & & & & 4.51 & 8.38 & 11.82 & 14.94 & 17.82 \\
\hline Ag20 & & $8.4 e-4$ & & & $7.5 e-4$ & $6.7 e-4$ & $5.9 \mathrm{e}-4$ & $5.0 \mathrm{e}-4$ & $4.2 \mathrm{e}-4$ & $5.7 e-4$ & $5.0 \mathrm{e}-4$ & $4.3 e-4$ & $3.6 e-4$ & $3.0 e-4$ \\
\hline $\mathrm{Al}$ & & & & 1.88 & 0.19 & 0.38 & 0.56 & 0.75 & 0.94 & 0.15 & 0.29 & 0.42 & 0.56 & 0.69 \\
\hline $\mathrm{Al}(\mathrm{OH}) 3$ & & & & 1.15 & 0.12 & 0.23 & 0.35 & 0.46 & 0.58 & 0.09 & 0.18 & 0.26 & 0.34 & 0.42 \\
\hline $\mathrm{Al} 203$ & 0.45 & 10.16 & & 0.39 & 9.19 & 8.21 & 7.23 & 6.25 & 5.28 & 7.00 & 6.15 & 5.34 & 4.56 & 3.81 \\
\hline As & $2.0 e-4$ & & & & 0 & 0 & 0 & 0 & 0 & $3.9 e-5$ & $3.4 e-5$ & $2.9 e-5$ & $2.5 e-5$ & $2.0 \mathrm{e}-5$ \\
\hline As20 & & $1.2 \mathrm{e}-3$ & & & $1.1 \mathrm{e}-3$ & $9.5 e-4$ & $8.3 e-4$ & $7.1 e-4$ & $6.0 e-4$ & $8.1 e-4$ & $7.0 \mathrm{e}-4$ & $6.1 \mathrm{e}-4$ & $5.1 e-4$ & $4.2 e-4$ \\
\hline $\mathrm{BaO}$ & & $9.8 \mathrm{e}-3$ & & & $8.8 \mathrm{e}-3$ & $7.8 \mathrm{e}-3$ & $6.8 \mathrm{e}-3$ & $5.9 \mathrm{e}-3$ & $4.9 \mathrm{e}-3$ & $6.6 e-3$ & $5.8 e-3$ & $5.0 \mathrm{e}-3$ & $4.2 e-3$ & $3.5 e-3$ \\
\hline C & & & & 21.62 & 2.16 & 4.32 & 6.49 & 8.65 & 10.81 & 1.75 & 3.33 & 4.88 & 6.40 & 7.91 \\
\hline $\mathrm{Ca}(\mathrm{OH}) 2$ & & & & 0.66 & 0.07 & 0.13 & 0.20 & 0.26 & 0.33 & 0.05 & 0.10 & 0.15 & 0.20 & 0.24 \\
\hline $\mathrm{CaCO} 3$ & & 12.69 & & & 11.42 & 10.15 & 8.88 & 7.61 & 6.35 & 8.59 & 7.50 & 6.47 & 5.48 & 4.52 \\
\hline $\mathrm{CaO}$ & 96.00 & 0.11 & & 2.31 & 0.33 & 0.55 & 0.77 & 0.99 & 1.21 & 19.17 & 16.83 & 14.63 & 12.51 & 10.46 \\
\hline $\mathrm{CdO}$ & & $5.6 e-5$ & & & $5.0 \mathrm{e}-5$ & $4.5 e-5$ & $3.9 e-5$ & $3.3 e-5$ & $2.8 e-5$ & $3.8 \mathrm{e}-5$ & $3.3 e-5$ & $2.8 \mathrm{e}-5$ & $2.4 e-5$ & 2.0e-5 \\
\hline $\mathrm{CeO} 2$ & & $5.4 \mathrm{e}-3$ & & & $4.9 \mathrm{e}-3$ & $4.3 e-3$ & $3.8 \mathrm{e}-3$ & $3.2 \mathrm{e}-3$ & $2.7 e-3$ & $3.7 e-3$ & $3.2 \mathrm{e}-3$ & $2.8 \mathrm{e}-3$ & $2.3 e-3$ & $1.9 \mathrm{e}-3$ \\
\hline $\mathrm{Cl}$ & & $3.9 \mathrm{e}-3$ & & 4.87 & 0.49 & 0.98 & 1.46 & 1.95 & 2.44 & 0.40 & 0.75 & 1.10 & 1.44 & 1.78 \\
\hline $\mathrm{CO} 2$ & 0.80 & & & & 0 & 0 & o & 0 & 0 & 0.16 & 0.14 & 0.12 & 0.10 & 0.08 \\
\hline $\mathrm{Cr}$ & & & & 2.06 & 0.21 & 0.41 & 0.62 & 0.82 & 1.03 & 0.17 & 0.32 & 0.47 & 0.61 & 0.75 \\
\hline $\mathrm{Cr} 2 \mathrm{O} 3$ & & 0.03 & 0.13 & & 0.03 & 0.02 & 0.02 & 0.02 & 0.01 & 0.03 & 0.03 & 0.03 & 0.03 & 0.03 \\
\hline Cs2O & & $5.2 \mathrm{e}-3$ & & & $4.7 e-3$ & $4.1 \mathrm{e}-3$ & $3.6 e-3$ & $3.1 e-3$ & $2.6 e-3$ & $3.5 \mathrm{e}-3$ & $3.1 e-3$ & $2.6 e-3$ & $2.2 e-3$ & $1.8 e-3$ \\
\hline $\mathrm{Cu}$ & & & & 0.56 & 0.06 & 0.11 & 0.17 & 0.23 & 0.28 & 0.05 & 0.09 & 0.13 & 0.17 & 0.21 \\
\hline $\mathrm{CuO}$ & & $5.5 \mathrm{e}-3$ & 0.33 & & $4.9 e-3$ & $4.4 \mathrm{e}-3$ & $3.8 \mathrm{e}-3$ & $3.3 e-3$ & $2.7 \mathrm{e}-3$ & 0.02 & 0.03 & 0.04 & 0.05 & 0.06 \\
\hline$F$ & 0.03 & & & & 0 & 0 & 0 & 0 & 0 & $5.9 \mathrm{e}-3$ & 5.1e-3 & $4.4 \mathrm{e}-3$ & $3.7 e-3$ & $3.0 e-3$ \\
\hline $\mathrm{Fe}$ & & & & 30.92 & 3.09 & 6.18 & 9.28 & 12.37 & 15.46 & 2.50 & 4.76 & 6.98 & 9.16 & 11.32 \\
\hline $\mathrm{Fe}(\mathrm{OH}) 3$ & & & & 1.08 & 0.11 & 0.22 & 0.32 & 0.43 & 0.54 & 0.09 & 0.17 & 0.24 & 0.32 & 0.40 \\
\hline $\mathrm{Fe} 2 \mathrm{O} 3$ & 0.12 & & & 0.12 & 0.01 & 0.02 & 0.04 & 0.05 & 0.06 & 0.03 & 0.04 & 0.05 & 0.05 & 0.06 \\
\hline $\mathrm{Fe} 304$ & & 4.13 & 99.09 & & 3.72 & 3.30 & 2.89 & 2.48 & 2.06 & 7.26 & 10.75 & 13.82 & 16.59 & 19.13 \\
\hline
\end{tabular}


Table A-2. (continued)

\begin{tabular}{|c|c|c|c|c|c|c|c|c|c|c|c|c|c|c|}
\hline $\mathrm{H}$ & & & & 3.34 & 0.33 & 0.67 & 1.00 & 1.34 & 1.67 & 0.27 & 0.51 & 0.75 & 0.99 & 1.22 \\
\hline $\mathrm{H} 2 \mathrm{O}$ & & 9.86 & & 7.16 & 9.59 & 9.32 & 9.05 & 8.78 & 8.51 & 7.26 & 6.93 & 6.64 & 6.38 & 6.13 \\
\hline $\mathrm{Hf}$ & & & & $9.4 \mathrm{e}-4$ & $9.4 e-5$ & $1.9 \mathrm{e}-4$ & $2.8 \mathrm{e}-4$ & $3.8 \mathrm{e}-4$ & $4.7 e-4$ & $7.6 e-5$ & $1.4 e-4$ & $2.1 e-4$ & $2.8 e-4$ & $3.4 e-4$ \\
\hline $\mathrm{HgO}$ & & $1.1 \mathrm{e}-5$ & & & $9.5 e-6$ & $8.4 e-6$ & $7.4 e-6$ & $6.3 e-6$ & $5.3 e-6$ & $7.1 e-6$ & $6.2 e-6$ & $5.4 \mathrm{e}-6$ & $4.6 \mathrm{e}-6$ & $3.8 \mathrm{e}-6$ \\
\hline $\mathrm{K} 2 \mathrm{O}$ & & 2.36 & & 0.05 & 2.13 & 1.90 & 1.67 & 1.44 & 1.21 & 1.60 & 1.40 & 1.22 & 1.03 & 0.86 \\
\hline KNO3 & & & & 1.35 & 0.14 & 0.27 & 0.41 & 0.54 & 0.68 & 0.11 & 0.21 & 0.30 & 0.40 & 0.49 \\
\hline $\mathrm{KOH}$ & & & & 0.74 & 0.07 & 0.15 & 0.22 & 0.30 & 0.37 & 0.06 & 0.11 & 0.17 & 0.22 & 0.27 \\
\hline $\mathrm{Mg}(\mathrm{OH}) 2$ & & & & 0.79 & 0.08 & 0.16 & 0.24 & 0.32 & 0.40 & 0.06 & 0.12 & 0.18 & 0.23 & 0.29 \\
\hline $\mathrm{MgO}$ & 0.50 & 2.22 & & 0.06 & 2.00 & 1.79 & 1.57 & 1.36 & 1.14 & 1.60 & 1.41 & 1.22 & 1.04 & 0.86 \\
\hline$\overline{M n}$ & & & & $8.4 \mathrm{e}-4$ & $8.4 \mathrm{e}-5$ & $1.7 e-4$ & $2.5 \mathrm{e}-4$ & $3.4 e-4$ & $4.2 \mathrm{e}-4$ & $6.8 e-5$ & $1.3 \mathrm{e}-4$ & $1.9 e-4$ & $2.5 e-4$ & $3.1 \mathrm{e}-4$ \\
\hline $\mathrm{Mn} 203$ & & & & $8.1 e^{-4}$ & $8.1 e-5$ & $1.6 \mathrm{e}-4$ & $2.4 e-4$ & $3.2 \mathrm{e}-4$ & $4.1 e-4$ & $6.6 \mathrm{e}-5$ & $1.3 e-4$ & $1.8 \mathrm{e}-4$ & $2.4 e-4$ & $3.0 \mathrm{e}-4$ \\
\hline $\mathrm{MnO} 2$ & 0.05 & & 0.85 & & 0 & 0 & 0 & 0 & 0 & 0.05 & 0.08 & 0.11 & 0.13 & 0.16 \\
\hline Mo & & & & 0.04 & $4.4 c-3$ & $8.8 \mathrm{e}-3$ & 0.01 & 0.02 & 0.02 & $3.6 \mathrm{e}-3$ & $6.8 \mathrm{e}-3$ & $9.9 \mathrm{e}-3$ & 0.01 & 0.02 \\
\hline$N$ & & & & 0.06 & $5.8 \mathrm{e}-3$ & 0.01 & 0.02 & 0.02 & 0.03 & $4.7 e-3$ & $8.9 e-3$ & 0.01 & 0.02 & 0.02 \\
\hline $\mathrm{Na2O}$ & 0.03 & 1.25 & & 0.04 & 1.13 & 1.01 & 0.89 & 0.77 & 0.65 & 0.86 & 0.75 & 0.65 & 0.56 & 0.46 \\
\hline $\mathrm{Na} 2 \mathrm{SO} 4$ & & & & 0.15 & 0.01 & 0.03 & 0.04 & 0.06 & 0.07 & 0.01 & 0.02 & 0.03 & 0.04 & 0.05 \\
\hline $\mathrm{NaCl}$ & & & & 0.15 & 0.02 & 0.03 & 0.05 & 0.06 & 0.08 & 0.01 & 0.02 & 0.03 & 0.05 & 0.06 \\
\hline $\mathrm{NaNO} 3$ & & & & 2.70 & 0.27 & 0.54 & 0.81 & 1.08 & 1.35 & 0.22 & 0.42 & 0.61 & 0.80 & 0.99 \\
\hline $\mathrm{NaOH}$ & & & & 0.15 & 0.01 & 0.03 & 0.04 & 0.06 & 0.07 & 0.01 & 0.02 & 0.03 & 0.04 & 0.05 \\
\hline $\mathrm{Ni}$ & & & & 0.92 & 0.09 & 0.18 & 0.27 & 0.37 & 0.46 & 0.07 & 0.14 & 0.21 & 0.27 & 0.33 \\
\hline $\mathrm{NiO}$ & & $4.2 e-3$ & 0.19 & & $3.8 \mathrm{e}-3$ & $3.4 \mathrm{e}-3$ & $3.0 \mathrm{e}-3$ & $2.5 \mathrm{e}-3$ & $2.1 e-3$ & 0.01 & 0.02 & 0.02 & 0.03 & 0.04 \\
\hline 0 & & & & 7.63 & 0.76 & 1.53 & 2.29 & 3.05 & 3.82 & 0.62 & 1.18 & 1.72 & 2.26 & 2.79 \\
\hline P2OS & 0.02 & & & $2.3 e-3$ & $2.3 e-4$ & $4.6 \mathrm{e}-4$ & $7.0 \mathrm{e}-4$ & $9.3 e-4$ & $1.2 \mathrm{e}-3$ & $4.1 e-3$ & $3.8 \mathrm{e}-3$ & $3.5 e-3$ & $3.1 \mathrm{e}-3$ & $2.8 \mathrm{e}-3$ \\
\hline $\mathrm{Pb}$ & $5.0 e-4$ & & & 0.19 & 0.02 & 0.04 & 0.06 & 0.08 & 0.09 & 0.02 & 0.03 & 0.04 & 0.06 & 0.07 \\
\hline $\mathrm{PbO}$ & & $2.4 \mathrm{e}-3$ & & & $2.2 e-3$ & $1.9 \mathrm{e}-3$ & $1.7 \mathrm{e}-3$ & $1.5 \mathrm{e}-3$ & $1.2 \mathrm{e}-3$ & $1.6 \mathrm{e}-3$ & $1.4 \mathrm{e}-3$ & $1.2 \mathrm{e}-3$ & $1.0 \mathrm{e}-3$ & $8.6 e-4$ \\
\hline PO4 & & 0.28 & 0.04 & & 0.25 & 0.23 & 0.20 & 0.17 & 0.14 & 0.19 & 0.17 & 0.15 & 0.13 & 0.11 \\
\hline$s$ & & & & 0.03 & $3.1 e-3$ & $6.1 \mathrm{e}-3$ & $9.2 \mathrm{e}-3$ & 0.01 & 0.02 & $2.5 \mathrm{e}-3$ & $4.7 e-3$ & $6.9 \mathrm{e}-3$ & $9.1 e-3$ & 0.01 \\
\hline $\mathrm{SeO}$ & & $1.2 \mathrm{e}-4$ & & & $1.1 \mathrm{e}-4$ & $9.4 \mathrm{e}-5$ & $8.2 e-5$ & $7.0 e-5$ & $5.9 \mathrm{e}-5$ & $7.9 \mathrm{e}-5$ & $6.9 \mathrm{e}-5$ & 6.0e-5 & $5.1 e-5$ & $4.2 e-5$ \\
\hline $\mathrm{Si}$ & & & & 0.01 & $1.4 e-3$ & $2.8 \mathrm{e}-3$ & $4.2 \mathrm{e}-3$ & $5.6 \mathrm{e}-3$ & $7.0 \mathrm{e}-3$ & $1.1 \mathrm{e}-3$ & $2.2 \mathrm{e}-3$ & $3.2 \mathrm{e}-3$ & $4.2 \mathrm{e}-3$ & $5.1 e-3$ \\
\hline $\mathrm{SiO} 2$ & 1.50 & 56.39 & 0.21 & 5.74 & 51.33 & 46.26 & 41.20 & 36.13 & 31.07 & 38.96 & 34.50 & 30.29 & 26.26 & 22.37 \\
\hline $\mathrm{SO} 3$ & 0.20 & & & 0.03 & $2.9 e-3$ & $5.8 \mathrm{e}-3$ & $8.8 \mathrm{e}-3$ & 0.01 & 0.01 & 0.04 & 0.04 & 0.04 & 0.03 & 0.03 \\
\hline SO4 & & 0.04 & & 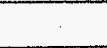 & 0.04 & 0.03 & 0.03 & 0.02 & 0.02 & 0.03 & 0.02 & 0.02 & 0.02 & 0.01 \\
\hline $\mathrm{TiO} 2$ & 0.05 & 0.42 & & $4.9 e-3$ & 0.38 & 0.34 & 0.30 & 0.25 & 0.21 & 0.29 & 0.26 & 0.22 & 0.19 & 0.16 \\
\hline $\mathrm{ZnO}$ & & $9.8 \mathrm{e}-3$ & & & $8.8 \mathrm{e}-3$ & $7.8 \mathrm{e}-3$ & $6.8 \mathrm{e}-3$ & $5.9 \mathrm{e}-3$ & $4.9 \mathrm{e}-3$ & $6.6 \mathrm{e}-3$ & $5.8 \mathrm{e}-3$ & $5.0 \mathrm{e}-3$ & $4.2 c-3$ & $3.5 e-3$ \\
\hline $\mathrm{Zr}$ & & & & 0.87 & 0.09 & 0.17 & 0.26 & 0.35 & 0.44 & 0.07 & 0.13 & 0.20 & 0.26 & 0.32 \\
\hline $\mathrm{ZrO2}$ & & $9.8 \mathrm{e}-3$ & & & $8.8 \mathrm{e}-3$ & $7.8 \mathrm{e}-3$ & $6.8 \mathrm{e}-3$ & $5.9 \mathrm{e}-3$ & $4.9 \mathrm{e}-3$ & $6.6 \mathrm{e}-3$ & $5.8 \mathrm{e}-3$ & $5.0 \mathrm{e}-3$ & $4.2 \mathrm{e}-3$ & $3.5 e-3$ \\
\hline & & & & & & & & & & & & & & \\
\hline Total & 99.8 & 100.0 & 100.8 & 99.8 & 100.0 & 100.0 & 100.0 & 99.9 & 99.9 & 100.0 & 100.0 & 100.0 & 100.1 & 100.1 \\
\hline
\end{tabular}




\section{Appendix B}

Calculated Proximate Analysis for the BWID Phase 2 Feed Mixtures 
, - 
Table B-1. Calculated proximate analysis of Nom-90 feed mixture.

\begin{tabular}{|c|c|c|c|c|c|c|c|c|c|c|c|c|c|c|c|c|c|}
\hline \multirow[b]{3}{*}{ Component } & \multirow[b]{3}{*}{ NOM-90 } & \multicolumn{15}{|c|}{ Composition (wt \%) } & \multirow[b]{3}{*}{ Total } \\
\hline & & \multirow{2}{*}{$\begin{array}{c}\text { Moisture } \\
\text { Loss }\end{array}$} & \multirow{2}{*}{\begin{tabular}{|c|} 
Volatile \\
Organics \\
Loss
\end{tabular}} & \multirow[b]{2}{*}{$\mathrm{H} 2 \mathrm{O}$} & \multirow{2}{*}{\multicolumn{3}{|c|}{ 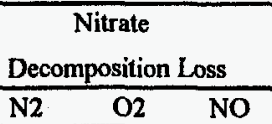 }} & & \multirow{2}{*}{\multicolumn{2}{|c|}{$\begin{array}{l}\text { Carbonate } \\
\text { Decomposition Loss } \\
\mathrm{CO} \quad \mathrm{CO} 2\end{array}$}} & \multicolumn{2}{|c|}{$\begin{array}{c}\text { Sulfate } \\
\text { Decomposition Loss } \\
\end{array}$} & \multicolumn{2}{|c|}{$\begin{array}{c}\text { Chloride } \\
\text { Evolution Loss } \\
\end{array}$} & \multirow{2}{*}{$\begin{array}{l}\text { Fixed } \\
\text { Carbon }\end{array}$} & \multirow{2}{*}{$\begin{array}{l}\text { Solid } \\
\text { Inerts }\end{array}$} & \\
\hline & & & & & & & & NO2 & & & $\mathrm{SO} 2$ & 02 & $\mathrm{HCl}$ & $\mathrm{Cl2}$ & & & \\
\hline & & & & & & & & & & & & & & & & & \\
\hline Ce spike & 0.50 & & & & & & & & & & & & & & & 100 & 100 \\
\hline Soil & 67.7 & 9.9 & & & & & & & & 5.58 & 0.03 & 0.01 & & 0.01 & & 84.5 & 100 \\
\hline Lime & 19.7 & & & & & & & & & 0.80 & 0.15 & 0.08 & & & & 99.0 & 100 \\
\hline Mill scale & 4.51 & & & & & & & & & & & & & & & 100 & 100 \\
\hline & & & & & & & & & & & & & & & & & \\
\hline Carbon steel & 1.71 & & & & & & & & & & & & & & 0.20 & 100 & 100 \\
\hline Stainless steel & 0.87 & & & & & & & & & & & & & & & 100 & 100 \\
\hline Aluminum & 0.14 & & & & & & & & & & & & & & & 100 & 100 \\
\hline Zirconium & 0.07 & & & & & & & & & & & & & & & 100 & 100 \\
\hline Copper & 0.04 & & & & & & & & & & & & & & & 100 & 100 \\
\hline Lead & 0.01 & & & & & & & & & & & & & & & 100 & 100 \\
\hline Polyethylene & 0.42 & & 100 & & & & & & & & & & & & & & 100 \\
\hline Wood pellets (paper) & 0.25 & 10.0 & 70.0 & & & & & & & & & & & & 20.0 & & 100 \\
\hline Wood pellets (wood) & 0.87 & 10.0 & 70.0 & & & & & & & & & & & & 20.0 & & 100 \\
\hline Rubber & 0.19 & & 100 & & & & & & & & & & & & & & 100 \\
\hline Oil Dri sorbent & 0.11 & 4.00 & & & & & & & & & & & & & & 96.0 & 100 \\
\hline Wood pellets (cloth) & 0.06 & 10.0 & 70.0 & & & & & & & & & & & & 20.0 & & 100 \\
\hline Texaco Regal Oil & 0.31 & & 100 & & & & & & & & & & & & & & 100 \\
\hline Hydraulic oil & 0.32 & & 100 & & & & & & & & & & & & & & 100 \\
\hline Wheel bearing grease & 0.04 & & 90.0 & & & & & & & & & & & & 10.0 & & 100 \\
\hline PVC & 0.52 & & 24.5 & & & & & & & & & & 47.7 & 10.3 & 17.5 & & 100 \\
\hline Microcel E & 0.45 & 10.0 & & & & & & & & & & & & & & 90.0 & 100 \\
\hline Oil Dri & 0.07 & 4.00 & & & & & & & & & & & & & & 96.0 & 100 \\
\hline NaNO3 & 0.20 & & & & 11.0 & 39.5 & 9.4 & 3.61 & & & & & & & & 36.5 & 100 \\
\hline $\mathrm{KNO3}$ & 0.10 & & & & 9.2 & 33.3 & 7.92 & 3.04 & & & & & & & & 46.7 & 100 \\
\hline $\mathrm{NaCl}$ & 0.01 & & & & & & & & & & & & 62.4 & & & 37.6 & 100 \\
\hline $\mathrm{Na2SO} 4$ & 0.01 & & & & & & & & & & 45.1 & 11.3 & & & & 43.6 & 100 \\
\hline $\mathrm{NaOH}$ & 0.01 & & & 22.5 & & & & & & & & & & & & 77.5 & 100 \\
\hline $\mathrm{H} 2 \mathrm{O}$ & 0.37 & 100 & & & & & & & & & & & & & & & 100 \\
\hline $\mathrm{Al}(\mathrm{OH}) 3$ & 0.09 & & & 34.6 & & & & & & & & & & & & 65.4 & 100 \\
\hline $\mathrm{Fe}(\mathrm{OH}) 3$ & 0.08 & & & 25.3 & & & & & & & & & & & & 74.7 & 100 \\
\hline $\mathrm{Ca}(\mathrm{OH}) 2$ & 0.05 & & & 24.3 & & & & & & & & & & & & 75.7 & 100 \\
\hline $\mathrm{Mg}(\mathrm{OH}) 2$ & 0.06 & & & 30.9 & & & & & & & & & & & & 69.1 & 100 \\
\hline $\mathrm{KOH}$ & 0.06 & & & 16.1 & & & & & & & & & & & & 83.9 & 100 \\
\hline Portland Cement & 0.09 & 1.49 & & & & & & & & & 1.95 & 0.97 & & & & 95.6 & 100 \\
\hline Mixture Averages & 100 & 7.22 & 2.22 & 0.09 & 0.03 & 0.11 & 0.03 & 0.01 & 0.00 & 3.94 & 0.06 & $\begin{array}{ll}0.03 \\
\end{array}$ & 0.25 & 0.06 & 0.33 & 85.6 & 100 \\
\hline
\end{tabular}


Table B-2. Calculated proximate analysis of Nom-80 feed mixture.

\begin{tabular}{|c|c|c|c|c|c|c|c|c|c|c|c|c|c|c|c|c|c|}
\hline \multirow[b]{3}{*}{ Component } & \multirow[b]{3}{*}{ NOM-80 } & \multirow{3}{*}{$\begin{array}{c}\text { Moisture } \\
\text { L.oss } \\
\end{array}$} & \multirow{3}{*}{$\begin{array}{c}\text { Volatile } \\
\text { Organics } \\
\text { Loss } \\
\end{array}$} & \multirow[b]{3}{*}{$\mathrm{H} 2 \mathrm{O}$} & \multicolumn{12}{|c|}{ Composition (wt \%) } & \multirow[b]{3}{*}{ Total } \\
\hline & & & & & \multicolumn{4}{|c|}{$\begin{array}{c}\text { Nitrate } \\
\text { Decomposition Loss }\end{array}$} & \multicolumn{2}{|c|}{\begin{tabular}{|c|} 
Carbonale \\
Decomposition Loss \\
\end{tabular}} & \multicolumn{2}{|c|}{$\begin{array}{c}\text { Sulfate } \\
\text { Decomposition Loss }\end{array}$} & \multicolumn{2}{|c|}{$\begin{array}{c}\text { Chloride } \\
\text { Evolution Las's }\end{array}$} & \multirow{2}{*}{$\begin{array}{c}\text { Fixed } \\
\text { Carbon }\end{array}$} & \multirow{2}{*}{$\begin{array}{c}\text { Solid } \\
\text { Inerts } \\
\end{array}$} & \\
\hline & & & & & $\mathrm{N} 2$ & $\mathrm{O} 2$ & No & NO2 & $\mathrm{Co}$ & $\mathrm{CO} 2$ & $\mathrm{SO} 2$ & 02 & $\mathrm{HCl}$ & $\mathrm{Cl} 2$ & & & \\
\hline Ce spike & 0.50 & & & & & & & & & & & & & & & 100 & 100 \\
\hline Soil & 59.1 & 9.9 & & & & & & & & 5.58 & 0.03 & 0.01 & & 0.01 & & 84.5 & 100 \\
\hline Lime & 17.1 & & & & & & & & & 0.80 & 0.15 & 0.08 & & & & 99.0 & 100 \\
\hline Mill scale & 8.38 & & & & & & & & & & & & & & & 100 & 100 \\
\hline & & & & & & & & & & & & & & & & & \\
\hline Carbon steel & 3.35 & & & & & & & & & & & & & & 0.20 & 100 & 100 \\
\hline Stainless steel & 1.70 & & & & & & & & & & & & & & & 100 & 100 \\
\hline Aluminum & 0.28 & & & & & & & & & & & & & & & 100 & 100 \\
\hline Zirconium & 0.14 & & & & & & & & & & & & & & & 100 & 100 \\
\hline Copper & 0.08 & & & & & & & & & & & & & & & 100 & 100 \\
\hline Lead & 0.03 & & & & & & & & & & & & & & & 100 & 100 \\
\hline Polyethylene & 0.83 & & 100 & & & & & & & & & & & & & & 100 \\
\hline Wood pellets (paper) & 0.48 & 10.0 & 70.0 & & & & & & & & & & & & 20.0 & & 100 \\
\hline Wood pellets (wood) & 1.71 & 10.0 & 70.0 & & & & & & & & & & & & 20.0 & & 100 \\
\hline Rubber & 0.37 & & 100 & & & & & & & & & & & & & & 100 \\
\hline Oil Dri sorbent & 0.22 & 4.00 & & & & & & & & & & & & & & 96.0 & 100 \\
\hline Wood pellets (cloth) & 0.11 & 10.0 & 70.0 & & & & & & & & & & & & 20.0 & & 100 \\
\hline Texaco Regal Oil & 0.60 & & 100 & & & & & & & & & & & & & & 100 \\
\hline Hydraulic oil & 0.63 & & 100. & & & & & & & & & & & & & & 100 \\
\hline Wheel bearing grease & 0.07 & & 90.0 & & & & & & & & & & & & 10.0 & & 100 \\
\hline PVC & 1.02 & & 24.5 & & & & & & & & & & 47.7 & 10.3 & 17.5 & & 100 \\
\hline Microcel E & 0.89 & 10.0 & & & & & & & & & & & & & & 90.0 & 100 \\
\hline Oil Dri & 0.13 & 4.00 & & & & & & & & & & & & & & 96.0 & 100 \\
\hline NaNO3 & 0.40 & & & & 11.0 & 39.5 & 9.4 & 3.61 & & & & & & & & 36.5 & 100 \\
\hline KNO3 & 0.20 & & & & 9.2 & 33.3 & 7.92 & 3.04 & & & & & & & & 46.7 & $\mathbf{1 0 0}$ \\
\hline $\mathrm{NaCl}$ & 0.02 & & & & & & & & & & & & 62.4 & & & 37.6 & 100 \\
\hline $\mathrm{Na2SO4}$ & 0.02 & & & & & & & & & & 45.1 & 11.3 & & & & 43.6 & 100 \\
\hline $\mathrm{NaOH}$ & 0.02 & & & 22.5 & & & & & & & & & & & & 77.5 & 100 \\
\hline 1120 & 0.73 & 100 & & & & & & & & & & & & & & & 100 \\
\hline $\mathrm{Al}(\mathrm{OH}) 3$ & 0.17 & & & 34.6 & & & & & & & & & & & & 65.4 & 100 \\
\hline $\mathrm{Fe}(\mathrm{OH})^{3}$ & 0.16 & & & 25.3 & & & & & & & & & & & & 74.7 & 100 \\
\hline $\mathrm{Ca}(\mathrm{OH}) 2$ & 0.10 & & & 24.3 & & & & & & & & & & & & 75.7 & $\begin{array}{l}-\quad 100 \\
\end{array}$ \\
\hline $\mathrm{Mg}(\mathrm{OH}) 2$ & 0.12 & & & 30.9 & & & & & & & & & & & & 69.1 & 100 \\
\hline $\mathrm{KOH}$ & 0.11 & & & 16.1 & & & & & & & & & & & & 83.9 & 100 \\
\hline Portland Cement & 0.17 & 1.49 & & & & & & & & & 1.95 & 0.97 & & & & 95.6 & 100 \\
\hline Mixture Averages & 100 & 6.90 & 4.36 & 0.18 & 0.06 & 0.23 & 0.05 & 0.02 & 0.00 & 3.44 & 0.06 & 0.03 & 0.50 & 0.11 & 0.65 & 83.4 & 100 \\
\hline
\end{tabular}


Table B-3. Calculated proximate analysis of Nom-70 feed mixture.

\begin{tabular}{|c|c|c|c|c|c|c|c|c|c|c|c|c|c|c|c|c|c|}
\hline \multirow[b]{3}{*}{ Component } & \multirow[b]{3}{*}{ NOM-70 } & \multicolumn{15}{|c|}{ Composition (wt \%) } & \multirow[b]{3}{*}{ Total } \\
\hline & & \multirow{2}{*}{$\begin{array}{c}\text { Moisture } \\
\text { Loss } \\
\end{array}$} & \multirow{2}{*}{$\begin{array}{c}\text { Volatile } \\
\text { Organics } \\
\text { Loss }\end{array}$} & \multirow[b]{2}{*}{$\mathrm{H} 2 \mathrm{O}$} & \multicolumn{4}{|c|}{$\begin{array}{c}\text { Nitrate } \\
\text { Decomposition Loss }\end{array}$} & \multicolumn{2}{|c|}{\begin{tabular}{|c|} 
Carbonate \\
Decomposition Loss \\
\end{tabular}} & \multicolumn{2}{|c|}{\begin{tabular}{|c|} 
Sulfate \\
Decomposition Loss \\
\end{tabular}} & \multicolumn{2}{|c|}{$\begin{array}{c}\text { Chloride } \\
\text { Evolution Loss }\end{array}$} & \multirow{2}{*}{$\begin{array}{c}\text { Fixed } \\
\text { Carbon }\end{array}$} & \multirow{2}{*}{$\begin{array}{l}\text { Solid } \\
\text { Inerts }\end{array}$} & \\
\hline & & & & & $\mathrm{N} 2$ & $\mathrm{O} 2$ & No & NO2 & $\mathrm{co}$ & $\mathrm{CO} 2$ & $\mathrm{SO} 2$ & $\mathrm{O} 2$ & $\mathrm{HCl}$ & $\mathrm{Cl} 2$ & & & \\
\hline & & & & & & & & & & & & & & & & & \\
\hline Ce spike & 0.50 & & & & & & & & & & & & & & & 100 & 100 \\
\hline Soil & 51.0 & 9.9 & & & & & & & & 5.58 & 0.03 & 0.01 & & 0.01 & & 84.5 & 100 \\
\hline Lime & 14.6 & & & & & & & & & 0.80 & 0.15 & 0.08 & & & & 99.0 & 100 \\
\hline Mill scale & 11.8 & & & & & & & & & & & & & & & 100 & 100 \\
\hline & & & & & & & & & & & & & & & & & \\
\hline Carbon steel & 4.96 & & & & 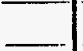 & $\ldots$ & & & & & & & & & 0.20 & 100 & 100 \\
\hline Stainless steel & 2.52 & & & & $\ldots$ & $\ldots$ & $\ldots$ & & & & & & & & & 100 & 100 \\
\hline Aluminum & 0.41 & & & & $\ldots$ & $\ldots$ & $\ldots$ & & & & & & & & & 100 & 100 \\
\hline Zirconium & 0.21 & & & & $\cdots$ & -1 & 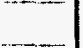 & & & & & & & & & 100 & 100 \\
\hline Copper & 0.12 & & & & $\ldots$ & $\ldots$ & 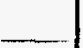 & & & & & & & & & 100 & 100 \\
\hline lead & 0.0 .4 & & & $\ldots$ & $\ldots$ & $\ldots$ &. & & & & & & & & & 100 & 100 \\
\hline Polyethylene & 1.23 & & 100 & 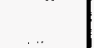 & . . & $\ldots$ & $\ldots$ & 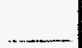 & & & & & & & & & 100 \\
\hline Wood pellets (paper) & 072 & 100 & 700 & & ... & $\ldots$ & & & & & & & & & 20.0 & & 100 \\
\hline Wood pellets (wood) & 254 & 100 & 700 & & & $\ldots \ldots$ & $\ldots$ & 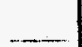 & & & & & & & 20.0 & & 100 \\
\hline Rubber & 035 & & 1000 & $=$ & 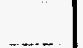 & 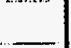 & 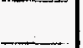 & & & & & & & & & & 100 \\
\hline Oil Dri sorhent & 0.31 & $4(0)$ & & $\ldots$ & $\ldots$ & $-\ldots$ & - & & & & & & & & -1 & 96.0 & 100 \\
\hline Wood pellets (cloth) & 0.17 & 100 & 70.0 & 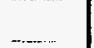 & $\ldots$ & $\ldots$ & - & & & & & & & & 20.0 & & 100 \\
\hline Texaco Regal Oil & 089 & & 100 & & 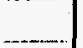 & $\underline{-}$ & 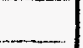 & & & & & & & & & & 100 \\
\hline Ilydraulic oil & 0.93 & & 100 & $\ldots$ & 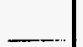 & $\ldots$ & & & & & & & & & 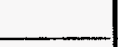 & & 100 \\
\hline Wheel bearing grease & 0.11 & & 90.0 & & & & & & & & & & & & 10.0 & & 100 \\
\hline PVC & 1.51 & & 24.5 & & & & & & & & & & 47.7 & 10.3 & 17.5 & & 100 \\
\hline Microcel E & 1.32 & 10.0 & & & & & & & & & & & & & & 90.0 & 100 \\
\hline Oil Dri & 0.20 & 4.00 & & & & & & & & & & & & & & 96.0 & 100 \\
\hline $\mathrm{NaNO} 3$ & 0.60 & & & & 11.0 & 39.5 & 9.4 & 3.61 & & & & & & & & 36.5 & 100 \\
\hline KNO3 & 0.30 & & & & 9.2 & 33.3 & 7.92 & 3.04 & & & & & & & $\therefore$ & 46.7 & 100 \\
\hline $\mathrm{NaCl}$ & 0.03 & & & & & & & & & & & & 62.4 & & & 37.6 & 100 \\
\hline $\mathrm{Na} 2 \mathrm{SO} 4$ & 0.03 & & & & & & & & & & 45.1 & 11.3 & & & & 43.6 & 100 \\
\hline $\mathrm{NaOH}$ & 0.03 & & & 22.5 & & & & & & & & & & & & 77.5 & 100 \\
\hline 1120 & 1.08 & 100 & & & & & & & & & & & & & & . & 100 \\
\hline $\mathrm{A}(\mathrm{OH}) 3$ & 0.25 & & & 34.6 & & & & & & & & & & & & 65.4 & 100 \\
\hline $\mathrm{Fe}(\mathrm{OH}) 3$ & 0.24 & & & 25.3 & & & & & & & & & & & & 74.7 & 100 \\
\hline $\mathrm{Ca}(\mathrm{OH}) 2$ & 0.15 & & & 24.3 & & & & & & & & & & & & 75.7 & 100 \\
\hline $\mathrm{Mg}(\mathrm{OH}) 2$ & 0.17 & & & 30.9 & & & & & & & & & & & & 69.1 & 100 \\
\hline KOH & 0.16 & & & 16.1 & & & & & & & & & & & & 83.9 & 100 \\
\hline Portland Cement & 0.26 & 1.49 & & & & & & & & & 1.95 & 0.97 & & & & 95.6 & 100 \\
\hline Mixture Averages & 100 & 6.61 & 6.46 & 0.27 & 0.09 & 0.33 & 0.08 & 0.03 & 0.00 & 2.96 & 0.06 & 0.02 & 0.74 & 0.16 & 0.97 & 81.2 & 100 \\
\hline
\end{tabular}


Table B-4. Calculated proximate analysis of Nom-60 feed mixture.

\begin{tabular}{|c|c|c|c|c|c|c|c|c|c|c|c|c|c|c|c|c|c|}
\hline \multirow[b]{3}{*}{ Component } & \multirow[b]{3}{*}{ NOM-60 } & \multirow{3}{*}{$\begin{array}{c}\text { Moisture } \\
\text { Loss } \\
\end{array}$} & \multicolumn{14}{|c|}{ Composition (wt \%) } & \multirow[b]{3}{*}{ Total } \\
\hline & & & \multirow{2}{*}{$\begin{array}{c}\text { Volatile } \\
\text { Organics } \\
\text { Loss } \\
\end{array}$} & \multirow[b]{2}{*}{$\mathrm{H} 2 \mathrm{O}$} & \multicolumn{4}{|c|}{$\begin{array}{c}\text { Nitrate } \\
\text { Decomposition Loss }\end{array}$} & \multicolumn{2}{|c|}{\begin{tabular}{|c|} 
Carbonate \\
Decomposition Loss \\
\end{tabular}} & \multicolumn{2}{|c|}{$\begin{array}{c}\text { Sulfate } \\
\text { Decomposition Loss }\end{array}$} & \multicolumn{2}{|c|}{$\begin{array}{c}\text { Chloride } \\
\text { Evolution Loss } \\
\end{array}$} & \multirow{2}{*}{$\begin{array}{c}\text { Fixed } \\
\text { Carbon }\end{array}$} & \multirow{2}{*}{$\begin{array}{l}\text { Solid } \\
\text { Inerts } \\
\end{array}$} & \\
\hline & & & & & $\mathrm{N} 2$ & 02 & NO & $\mathrm{NO2}$ & $\mathrm{CO}$ & $\mathrm{CO} 2$ & $\mathrm{SO} 2$ & $\mathbf{O 2}$ & $\mathrm{HCl}$ & $\mathrm{Cl} 2$ & & & \\
\hline Ce spike & 0.50 & & & & & & & & & & & & & & & 100 & 100 \\
\hline Soil & 43.2 & 9.9 & & & & & & & & 5.58 & $\mathbf{0 . 0 3}$ & 0.01 & & 0.01 & & 84.5 & 100 \\
\hline Lime & 12.3 & & & & & & & & & 0.80 & 0.15 & 0.08 & & & & 99.0 & 100 \\
\hline Mill scale & 14.9 & & & & & & & & & & & & & & & 100 & 100 \\
\hline & & & & & & & & & & & & & & & & & \\
\hline Carbon steel & 6.55 & & & & & & & & & & & & & & 0.20 & 100 & 100 \\
\hline Stainless steel & 3.33 & & & & & & & & & & & & & & & 100 & 100 \\
\hline Aluminum & 0.55 & & & & & & & & & & & & & & & 100 & 100 \\
\hline Zirconium & 0.27 & & & & & & & & & & & & & & & 100 & 100 \\
\hline Copper & 0.16 & & & & & & & & & & & & & & & 100 & 100 \\
\hline lead & 0.05 & & & & & & & & & & & & & & & 100 & 100 \\
\hline Polyethylene & 1.62 & & 100 & & & & & & & & & & & & & & 100 \\
\hline Wood pellets (paper) & 0.95 & 10.0 & 70.0 & & & & & & & & & & & & 20.0 & & 100 \\
\hline Wood pellets (wond) & 3.35 & 100 & 70.0 & & & & & & & & & & & & 20.0 & & 100 \\
\hline Rubber & 0.73 & - & 100 & & & & & & & & & & & & & & 100 \\
\hline Oil Dri sorbent & 0.44 & 4.00 & & & & & & & & & & & & & 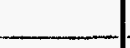 & 96.0 & 100 \\
\hline Wood pellets (cloth) & 0.22 & 10.0 & 70.0 & & & & & & & & & & & & 20.0 & & 100 \\
\hline Texaco Regal Oil & 1.18 & & 100 & & & & & & & & & & & & & & 100 \\
\hline IIydraulic oil & 1.22 & & 100 & & & & & & & & & & & & & & 100 \\
\hline Wheel bearing grease & 0.14 & & 90.0 & & & & & & & & & & & & 10.0 & & 100 \\
\hline PVC & 1.99 & & 24.5 & & & & & & & & & & 47.7 & 10.3 & 17.5 & & 100 \\
\hline Microcel l: & 1.75 & 10.0 & & & & & & & & & & & & & & 90.0 & 100 \\
\hline Oil Dri & 0.26 & 4.00 & & & & & & & & & & & & & & 96.0 & 100 \\
\hline $\mathrm{NaNO3}$ & 0.79 & & & & 11.0 & 39.5 & 9.4 & 3.61 & & & & & & & & 36.5 & 100 \\
\hline KNO3 & 0.39 & & & & 9.2 & 33.3 & 7.92 & 3.04 & & & & & & & & 46.7 & 100 \\
\hline $\mathrm{NaCl}$ & 0.04 & & & & & & & & & & & & 62.4 & & & 37.6 & 100 \\
\hline $\mathrm{Na2SO} 4$ & 0.04 & & & & & & & & & & 45.1 & 11.3 & & & & 43.6 & 100 \\
\hline $\mathrm{NaOH}$ & 0.04 & & & 22.5 & & & & & & & & & & & & 77.5 & 100 \\
\hline 1120 & 1.42 & 100 & & & & & & & & & & & & & & & 100 \\
\hline $\mathrm{Al}(\mathrm{OH}) 3$ & $\mathbf{0 . 3 3}$ & & & 34.6 & & & & & & & & & & & & 65.4 & 100 \\
\hline $\mathrm{Fe}(\mathrm{OH}) 3$ & 0.31 & & & 25.3 & & & & & & & & & & & & 74.7 & 100 \\
\hline $\mathrm{Ca}(\mathrm{OH}) 2$ & 0.19 & & & 24.3 & & & & & & & & & & & & 75.7 & 100 \\
\hline $\mathrm{Mg}(\mathrm{OH}) 2$ & 0.23 & & & 30.9 & & & & & & & & & & & & 69.1 & 100 \\
\hline KOH & 0.22 & & & 16.1 & & & & & & & & & & & & 83.9 & 100 \\
\hline Portland Cement & 0.34 & 1.49 & & & & & & & & & 1.95 & 0.97 & & & & 95.6 & 100 \\
\hline Mlxture Averages & 100 & 6.34 & 8.53 & 0.36 & 0.12 & 0.44 & 0.11 & 0.04 & 0.00 & 2.51 & 0.06 & 0.02 & 0.98 & 0.21 & 1.28 & 79.0 & 100 \\
\hline
\end{tabular}


Table B-5. Calculated proximate analysis of Nom-50 feed mixture.

\begin{tabular}{|c|c|c|c|c|c|c|c|c|c|c|c|c|c|c|c|c|c|}
\hline & \multicolumn{16}{|c|}{ Composition (wt \%) } & \multirow[b]{3}{*}{ Total } \\
\hline & & \multirow{2}{*}{$\begin{array}{c}\text { Moisture } \\
\text { Loss }\end{array}$} & \multirow{2}{*}{$\begin{array}{c}\text { Volatile } \\
\text { Organics } \\
\text { Loss } \\
\end{array}$} & \multirow[b]{2}{*}{$\mathrm{H} 2 \mathrm{O}$} & \multicolumn{4}{|c|}{$\begin{array}{c}\text { Nitrate } \\
\text { Decomposition Loss } \\
\end{array}$} & \multicolumn{2}{|c|}{\begin{tabular}{|c|} 
Carbonate \\
Decomposition Loss \\
\end{tabular}} & \multicolumn{2}{|c|}{$\begin{array}{c}\text { Sulfate } \\
\text { Decomposition Loss } \\
\end{array}$} & \multicolumn{2}{|c|}{$\begin{array}{c}\text { Chloride } \\
\text { Evolution Loss } \\
\end{array}$} & \multirow{2}{*}{$\begin{array}{c}\text { Fixed } \\
\text { Carbon } \\
\end{array}$} & \multirow{2}{*}{$\begin{array}{l}\text { Solid } \\
\text { Inerts }\end{array}$} & \\
\hline & & & & & \multirow{2}{*}{ N2 } & \multirow[t]{2}{*}{$\mathrm{O} 2$} & \multirow[t]{2}{*}{ No } & NO2 & co & $\mathrm{CO} 2$ & $\mathrm{SO} 2$ & 02 & $\mathrm{HCl}$ & $\mathrm{Cl} 2$ & & & \\
\hline Component & NOM-50 & & & & & & & & & & & & & & & & \\
\hline Ce spike & 0.50 & & & & & & & & & & & & & & & 100 & 100 \\
\hline Soil & 35.6 & 9.9 & & & & & & & & 5.58 & 0.03 & 0.01 & & 0.01 & & 84.5 & 100 \\
\hline Lime & 10.0 & & & & & & & & & 0.80 & 0.15 & 0.08 & & & & 99.0 & 100 \\
\hline Mill scale & 17.8 & & & & & & & & & & & & & & & 100 & 100 \\
\hline & & & & & & & & & & & & & & & & & \\
\hline Carbon steel & 8.12 & & & & & & & & & & & & & & 0.20 & 100 & 100 \\
\hline Stainless steel & 4.13 & & 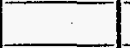 & & & & & & & & & & & & & 100 & 100 \\
\hline Aluminum & 0.68 & & & & & & & & & & & & & & & 100 & 100 \\
\hline Zirconium & 0.34 & & & & & & & & & & & & & & & 100 & 100 \\
\hline Copper & 0.20 & & & & & & & & & & & & & & & 100 & 100 \\
\hline Lead & 0.07 & & & & & & & & & & & & & & & 100 & 100 \\
\hline Polyethylene & 2.01 & & 100 & & & & & & & & & & & & & & 100 \\
\hline Wood pellets (paper) & 1.17 & 10.0 & 70.0 & & & & & & & & & & & & 20.0 & & 100 \\
\hline Wood pellets (wood) & 4.15 & 10.0 & 70.0 & & & & & & & & & & & & 20.0 & & 100 \\
\hline Rubber & 0.90 & & 100 & & & & & & & & & & & & & & 100 \\
\hline Oil Dri sorbent & 0.54 & 4.00 & & & & & & & & & & & & & & 96.0 & 100 \\
\hline Wood pellets (cloth) & 0.27 & 10.0 & 70.0 & & & & & & & & & & & & 20.0 & & 100 \\
\hline Texaco Regal Oil & 1.46 & & 100 & & & & & & & & & & & & & & 100 \\
\hline Hydraulic oil & 1.52 & & 100. & & & & & & & & & & & & . & & 100 \\
\hline Wheel hearing grease & 0.18 & & 90.0 & & & & & & & & & & & & 10.0 & & 100 \\
\hline PVC & 2.46 & & 24.5 & & & & & & & & & & 47.7 & 10.3 & 17.5 & & 100 \\
\hline Microcel E & 2.17 & 10.0 & & & & & & & & & & & & & . & 90.0 & 100 \\
\hline Oil Dri & 0.32 & 4.00 & & & & & & & & & & & & & & 96.0 & 100 \\
\hline $\mathrm{NaNO} 3$ & 0.97 & & & & 11.0 & 39.5 & 9.4 & 3.61 & & & & & & & & 36.5 & 100 \\
\hline KNO3 & 0.49 & & & & 9.2 & 33.3 & 7.92 & 3.04 & & & & & & & & 46.7 & 100 \\
\hline $\mathrm{NaCl}$ & 0.06 & & & & & & & & & & & & 62.4 & & & 37.6 & 100 \\
\hline $\mathrm{Na2SO} 4$ & 0.05 & & & & & & & & & & 45.1 & 11.3 & & & & 43.6 & 100 \\
\hline $\mathrm{NaOH}$ & 0.05 & & & 22.5 & & & & & & & & & & & & 77.5 & 100 \\
\hline 1120 & 1.77 & 100 & & & & & & & & & & & & & & & 100 \\
\hline Al(OH) 3 & 0.42 & & & 34.6 & & & & & & & & & & & & 65.4 & 100 \\
\hline $\mathrm{Fe}(\mathrm{OH}) 3$ & 0.39 & & & 25.3 & & & & & & & & & & & & 74.7 & 100 \\
\hline $\mathrm{Ca}(\mathrm{OH}) 2$ & 0.24 & & & 24.3 & & & & & & & & & & & & 75.7 & 100 \\
\hline $\mathrm{Mg}(\mathrm{OH}) 2$ & 0.29 & & & 30.9 & & & & & & & & & & 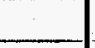 & & 69.1 & 100 \\
\hline KOH & 0.27 & & & 16.1 & & & & & & & & & & & & 83.9 & 100 \\
\hline Portland Cement & 0.42 & 1.49 & & & & & & & & & 1.95 & 0.97 & & & & 95.6 & 100 \\
\hline Mixture Averages & 100 & 6.09 & 10.6 & 0.44 & 0.15 & 0.55 & 0.13 & 0.05 & 0.00 & 2.07 & 0.06 & 0.02 & 1.21 & 0.26 & 1.58 & 76.8 & 100 \\
\hline
\end{tabular}


Table B-6. Calculated proximate analysis of Nom-0 feed mixture.

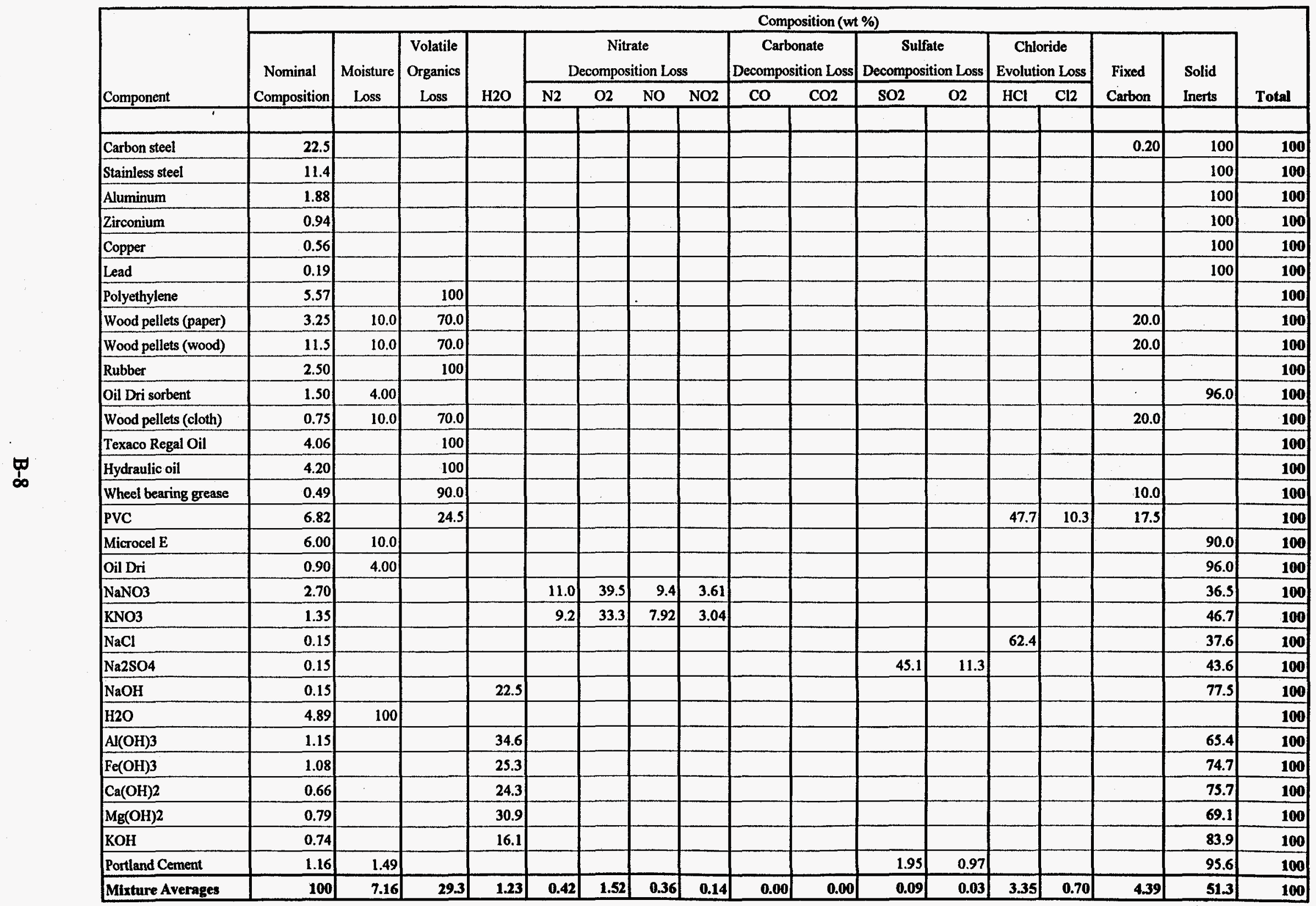




\section{Appendix C}

Estimated Offgas Compositions and Flowrates 


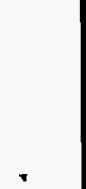

-

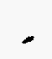

C-2 
Table C-1. Estimated offgas composition and flowrates.

\begin{tabular}{|c|c|c|c|c|c|c|c|c|c|c|c|c|c|}
\hline \multirow[b]{2}{*}{ Parameter } & & \multicolumn{2}{|c|}{ NOM-90 } & \multicolumn{2}{|c|}{ NOM-80 } & \multicolumn{2}{|c|}{ NOM-70 } & \multicolumn{2}{|c|}{ NOM-60 } & \multicolumn{2}{|c|}{ NOM-50 } & \multicolumn{2}{|c|}{ NOM-0 } \\
\hline & & $\begin{array}{c}\text { Flowrate } \\
\text { scfm }\end{array}$ & $\begin{array}{l}\text { Comp- } \\
\text { osition } \\
\end{array}$ & $\begin{array}{c}\text { Flowrate } \\
\text { scfm }\end{array}$ & $\begin{array}{l}\text { Comp- } \\
\text { osition }\end{array}$ & $\begin{array}{c}\text { Flowrale } \\
\text { scfm }\end{array}$ & $\begin{array}{l}\text { Comp- } \\
\text { osition }\end{array}$ & $\begin{array}{c}\text { Flowrate } \\
\text { scfm }\end{array}$ & $\begin{array}{l}\text { Comp- } \\
\text { osition }\end{array}$ & $\begin{array}{c}\text { Flowrate } \\
\text { scfm }\end{array}$ & $\begin{array}{l}\text { Comp- } \\
\text { osition }\end{array}$ & $\begin{array}{c}\text { Flowrate } \\
\text { scfm }\end{array}$ & $\begin{array}{l}\text { Comp- } \\
\text { osition }\end{array}$ \\
\hline \multicolumn{14}{|l|}{ FURNACE } \\
\hline \multicolumn{14}{|c|}{ Offgas Composition } \\
\hline $02, \%$ & & 1.67 & 1.77 & 1.20 & 0.90 & 0.87 & 0.51 & 1.70 & 0.79 & 2.59 & 1.00 & 1.26 & 0.20 \\
\hline $\mathrm{CO} 2, \%$ & & 8.30 & 8.79 & 10.0 & 7.53 & 11.8 & 6.84 & 13.5 & 6.25 & 15.2 & 5.86 & 33.7 & 5.36 \\
\hline $\mathrm{CO}, \mathrm{ppm}$ & & 8.06 & 85,309 & 12.2 & 91,823 & 16.3 & 94,897 & 20.3 & 94,328 & 24.3 & 93,814 & 60.7 & 96,726 \\
\hline $\mathrm{NO}, \mathrm{ppm}$ & & 0.06 & 620 & 0.12 & 865 & 0.17 & 992 & 0.22 & 1,044 & 0.28 & 1,077 & 0.77 & 1,231 \\
\hline \multicolumn{2}{|l|}{ NO2, ppm } & 0.01 & 155 & 0.03 & 216 & 0.04 & 248 & 0.06 & 261 & 0.07 & 269 & 0.19 & 308 \\
\hline N2, \% & & 39.6 & 419 & 63.3 & 47.5 & 87.1 & 50.7 & 115 & 53.3 & 143 & 55.0 & 360 & 57.4 \\
\hline $\mathrm{SO} 2, \mathrm{ppm}$ & & 006 & 589 & 0.06 & 420 & 0.06 & 328 & 0.06 & 264 & 0.06 & 222 & 0.09 & 143 \\
\hline $\mathrm{HCl}, \mathrm{ppm}$ & & 045 & 4.737 & 0.88 & 6.605 & 1.30 & 7.579 & 1.72 & 7,977 & 2.13 & 8.226 & 5.90 & 9,407 \\
\hline Cl2. ppm & & 0.05 & 545 & 0.10 & 734 & 0.14 & 832 & 0.19 & 870 & 0.23 & 894 & 0.64 & 1,015 \\
\hline H2O. $\%$ & & 312 & 330 & 35.2 & 26.5 & 39.3 & 22.9 & 43.4 & 20.1 & 47.5 & 18.3 & 96.9 & 15.4 \\
\hline \multicolumn{2}{|c|}{$\mathrm{THC}$ as $\mathrm{CH}^{2}$. ppm } & 50 & 53.777 & 10.0 & 74.995 & 14.8 & 86,046 & 19.5 & 90,563 & 24.2 & 93,399 & 67.0 & 106,805 \\
\hline & Toual & 945 & & 133 & & 172 & & 215 & & 259 & & 628 & \\
\hline \multicolumn{2}{|c|}{ Offgas Flowrate, scfm } & & 94.5 & & 133 & & 172 & & 215 & & 259 & & 628 \\
\hline & acim & & $370^{-}$ & & 522 & & 673 & & 844 & & 1,015 & & 2,459 \\
\hline & & & & & & & & & & & & & \\
\hline \multicolumn{2}{|c|}{ Offgas molecular weight, wt. basis } & & 25.5 & & 25.7 & & 25.7 & & 25.9 & & 26.0 & & 26.0 \\
\hline \multicolumn{2}{|c|}{ Offgas Flowrate, lb/hr } & & 376 & & 532 & & 689 & & 868 & & 1,048 & & 2,539 \\
\hline \multicolumn{2}{|c|}{ Offgas Flowrale, $\mathrm{kg} / \mathrm{hr}$} & & 170 & & 242 & & 313 & & 394 & & 476 & & 1,152 \\
\hline & & & & & & & & & & & & & \\
\hline \multicolumn{14}{|c|}{ Specified Parameters } \\
\hline & Feedrate, lb/hr & & 1,000 & & 1,000 & & 1,000 & & 1,000 & & 1,000 & & 1,000 \\
\hline & Offgas Temperature, $\mathrm{F}$ & & 1,600 & & 1,600 & & 1.600 & & 1,600 & & 1,600 & & 1,600 \\
\hline & Offgas Temperature, C & & 871 & & 871 & & 871 & & 871 & & 871 & & 871 \\
\hline & Static pressure, in. H2O & & -1.00 & & -1.00 & & -1.00 & & -1.00 & & -1.00 & & -1.00 \\
\hline & Air in-leakage, scfm & & 50.0 & & 50.0 & & 50.0 & & 50.0 & & 50.0 & & 50.0 \\
\hline & Air injected, scfm & & 0.00 & & 30.0 & & 60.0 & & 95.0 & & 130 & & 405 \\
\hline & $\%$ vol. organics to $\mathrm{CO}$ & & 25.0 & & 25.0 & & 25.0 & & 25.0 & & 25.0 & & 25.0 \\
\hline & $\%$ vol. organics to $\mathrm{CO} 2$ & & 25.0 & & 25.0 & & 25.0 & & 25.0 & & 25.0 & & 25.0 \\
\hline & \multicolumn{2}{|l|}{ Graphite Elec. Cons. (lb/hr) } & 7.00 & & 7.00 & & $\mathbf{7 . 0 0}$ & & 7.00 & & 7.00 & & 7.00 \\
\hline & & & & & & & & & & & & & \\
\hline \multirow[t]{3}{*}{ Notes: } & \multicolumn{6}{|c|}{ 1. All fixed C, including the graphite electrodes, is assumed to oxidize to CO. } & & & & & & & \\
\hline & 2. Thermal NOx is assum & ned to be ze & & & & & & & & & & & \\
\hline & & & & & & & & & & & & & \\
\hline
\end{tabular}


Table C-1. continued.

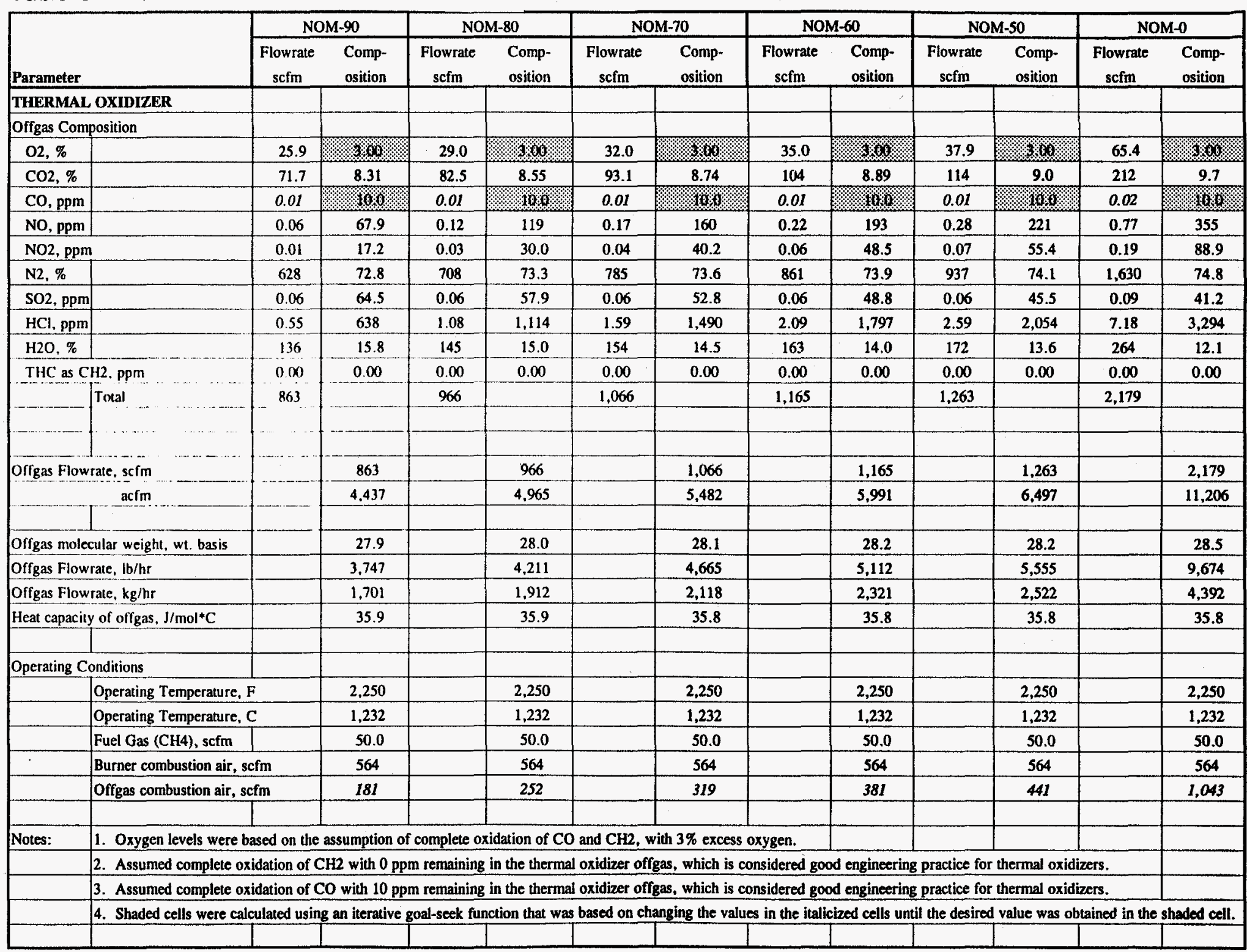


Table C-1. continued.

\begin{tabular}{|c|c|c|c|c|c|c|c|c|c|c|c|c|c|}
\hline & & & $1-90$ & & & & & NO! & & No & & & \\
\hline Parameter & & $\begin{array}{c}\text { Flowrate } \\
\text { scfm }\end{array}$ & $\begin{array}{l}\text { Comp- } \\
\text { osition }\end{array}$ & $\begin{array}{c}\text { Flowrate } \\
\text { scfm }\end{array}$ & $\begin{array}{l}\text { Comp- } \\
\text { osition }\end{array}$ & $\begin{array}{c}\text { Flowrate } \\
\text { scfm }\end{array}$ & $\begin{array}{l}\text { Comp- } \\
\text { osition }\end{array}$ & $\begin{array}{l}\text { Flowrate } \\
\text { scfm }\end{array}$ & $\begin{array}{l}\text { Comp- } \\
\text { osition }\end{array}$ & $\begin{array}{l}\text { Flowrate } \\
\text { scfm }\end{array}$ & $\begin{array}{l}\text { Comp- } \\
\text { osition }\end{array}$ & $\begin{array}{l}\text { Flowrate } \\
\text { scfm }\end{array}$ & $\begin{array}{l}\text { Comp- } \\
\text { osition }\end{array}$ \\
\hline SPRAY QUE & ENCH OUTLET & & & & & & & & & & & & \\
\hline Composition & & & & & & & & & & & & & \\
\hline $02, \%$ & & 25.9 & 1.82 & 29.0 & 1.82 & 32.0 & 1.82 & 35.0 & 1.82 & 37.9 & 1.82 & 65.4 & 1.82 \\
\hline $\mathrm{CO} 2 \%$ & & 71.7 & 5.04 & 82.5 & 5.18 & 93.1 & 5.30 & 104 & 5.39 & 114 & 5.47 & 212 & 5.90 \\
\hline $\mathrm{CO}, \mathrm{ppm}$ & & 0.01 & 6.06 & 0.01 & 6.06 & 0.01 & 6.06 & 0.01 & 6.06 & 0.01 & 6.05 & 0.02 & 6.07 \\
\hline No, ppm & & 0.06 & 41.1 & 0.12 & 72.3 & 0.17 & 97.0 & 0.22 & 117 & 0.28 & 134 & 0.77 & 215 \\
\hline NO2, ppm & & 0.01 & 10.4 & 0.03 & 18.2 & 0.04 & 24.4 & 0.06 & 29.4 & 0.07 & 33.6 & 0.19 & 53.9 \\
\hline $\mathrm{N} 2, \%$ & & 628 & 44.1 & 708 & 44.4 & 785 & 44.7 & 861 & 44.8 & 937 & 45.0 & 1,630 & 45.4 \\
\hline SO2, ppm & & 0.06 & 39.1 & 0.06 & 35.1 & 0.06 & 32.0 & 0.06 & 29.6 & 0.06 & 27.6 & 0.09 & 25.0 \\
\hline $\mathrm{HCl}, \mathrm{ppm}$ & & 0.55 & 387 & 1.08 & 675 & 1.59 & 904 & 2.09 & 1,090 & 2.59 & 1,246 & 7.18 & 1,999 \\
\hline $\mathrm{H} 2 \mathrm{O}, \%$ & & 697 & 49.0 & 772 & 48.5 & 846 & 48.1 & 919 & 47.8 & 991 & 47.6 & 1,676 & 46.7 \\
\hline THC as $\mathrm{CH}$ & $\mathrm{H} 2, \mathrm{ppm}$ & 0.00 & 0.00 & 0.00 & 0.00 & 0.00 & 0.00 & 0.00 & 0.00 & 0.00 & 0.00 & 0.00 & 0.00 \\
\hline & Total & 1.424 & & 1.593 & & 1,758 & & 1.921 & & 2,083 & & 3,591 & \\
\hline & & & & & & & & & & & & & \\
\hline Spray quench & h oudlet flowrate, scfm & & 1,424 & & 1,593 & & 1,758 & & 1,921 & & 2,083 & & 3,591 \\
\hline acfm & & & 2.625 & & 2,936 & & 3,241 & & 3,542 & & 3,840 & & 6,621 \\
\hline & & & & & & & & & & & & & \\
\hline Spray quench & h offgas mol. wt., wt. basi & & 24.0 & & 24.1 & & 24.1 & & 24.2 & & 24.2 & & 24.4 \\
\hline Spray quench & h offgas flowrate, $\mathrm{b} / \mathrm{h} / \mathrm{hr}$ & & 5,319 & & 5.968 & & 6,603 & & 7,230 & & 7,851 & & 13,631 \\
\hline Spray quench & h offgas flowrate, $\mathrm{kg} / \mathrm{hr}$ & & 2,415 & & 2,710 & & 2,998 & & 3,282 & & 3,564 & & 6,189 \\
\hline Heat capacity & y - spray quench offgas, J/ & $\mathrm{mol}^{*} \mathrm{C}$ & 33.1 & & 33.1 & & 33.0 & & 33.0 & & 33.0 & & 33.0 \\
\hline & & & & & & & & & & & & & \\
\hline Operating Co & onditions & & & & & & & & & & & & \\
\hline & Spray cooler outlet T (F) & & 500 & & 500 & & 500 & & 500 & & 500 & & 500 \\
\hline & Spray cooler outlet T (C) & & 260 & & 260 & & 260 & & 260 & & 260 & & 260 \\
\hline & Static pressure, in. $\mathrm{H} 2 \mathrm{O}$ & & -5.00 & & -5.00 & & -5.00 & & -5.00 & & -5.00 & & -5.00 \\
\hline & Added water (gal/min) & & 3.14 & & 3.51 & & 3.88 & & 4.24 & & 4.59 & & 7.91 \\
\hline
\end{tabular}


Table C-1. continued.

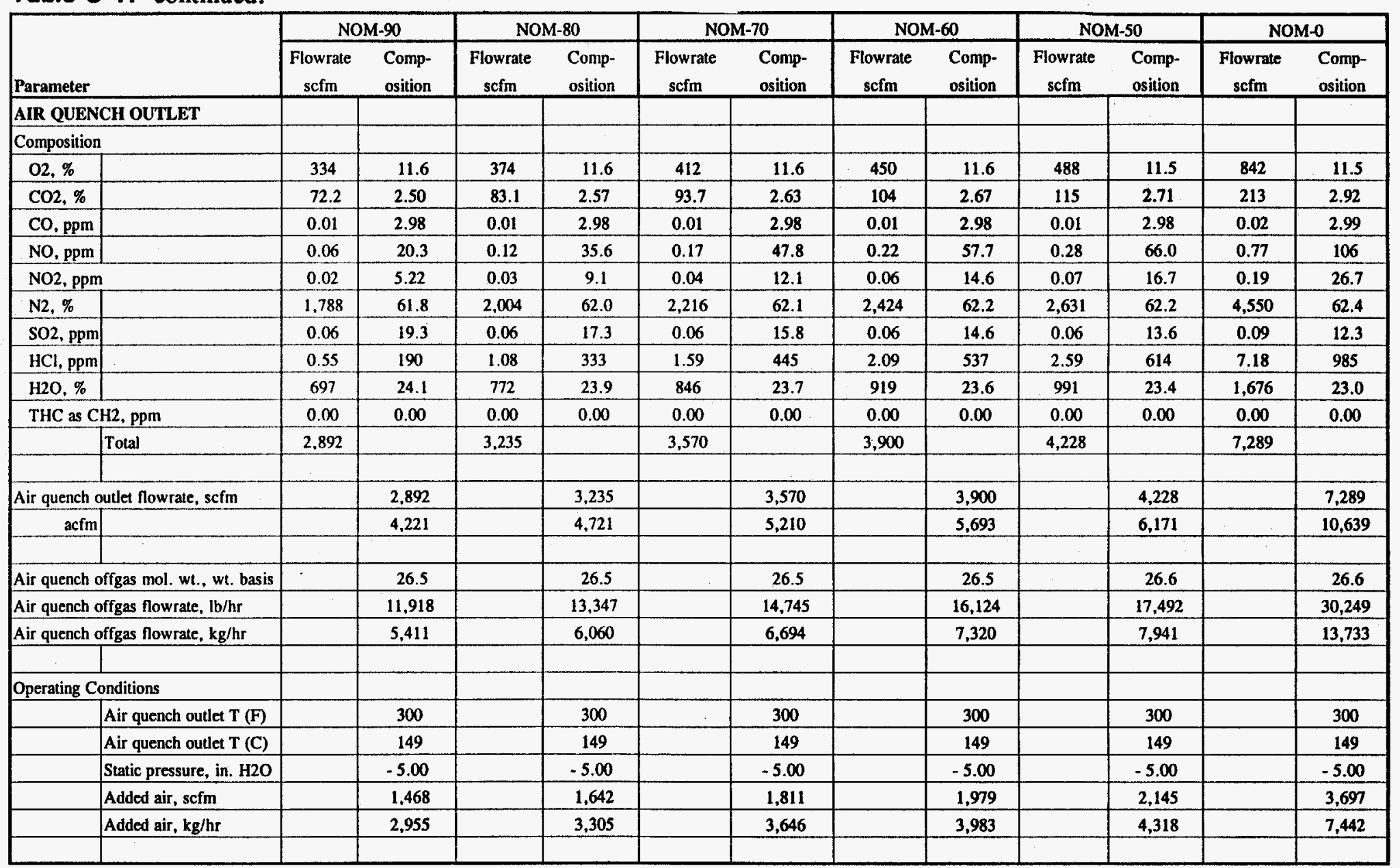


Table C-1. continued.

\begin{tabular}{|c|c|c|c|c|c|c|c|c|c|c|c|c|c|}
\hline & & & $1-90$ & NOI & & & & & & & & & \\
\hline Parameter & & $\begin{array}{c}\text { Flowrate } \\
\text { scfm }\end{array}$ & $\begin{array}{l}\text { Comp- } \\
\text { osition }\end{array}$ & $\begin{array}{c}\text { Flowrate } \\
\text { scfm }\end{array}$ & $\begin{array}{l}\text { Comp- } \\
\text { osition }\end{array}$ & $\begin{array}{c}\text { Flowrate } \\
\text { scfm }\end{array}$ & $\begin{array}{l}\text { Comp- } \\
\text { osition }\end{array}$ & $\begin{array}{c}\text { Flowrate } \\
\text { scfm }\end{array}$ & $\begin{array}{l}\text { Comp- } \\
\text { osition }\end{array}$ & $\begin{array}{c}\text { Flowrate } \\
\text { scfm }\end{array}$ & $\begin{array}{l}\text { Comp- } \\
\text { osition }\end{array}$ & $\begin{array}{c}\text { Flowrate } \\
\text { scfm }\end{array}$ & $\begin{array}{l}\text { Comp- } \\
\text { osition }\end{array}$ \\
\hline BAGHOUS & & & & & & & & & & & & & \\
\hline Offgas Com & position & & & & & & & & & & & & \\
\hline $02, \%$ & & 345 & 11.7 & 384 & 11.7 & 423 & 11.7 & 461 & 11.7 & 499 & 11.7 & 852 & 11.6 \\
\hline $\mathrm{CO} 2, \%$ & & 72.2 & 2.45 & 83.1 & 2.53 & 93.8 & 2.59 & 104 & 2.64 & 115 & 2.68 & 213 & 2.90 \\
\hline $\mathrm{CO}, \mathrm{ppm}$ & & 0.01 & 2.93 & 0.01 & 2.94 & 0.01 & 2.94 & 0.01 & 2.94 & 0.01 & 2.95 & 0.02 & 2.97 \\
\hline NO, ppm & & 0.06 & 19.9 & 0.12 & 35.1 & 0.17 & 47.1 & 0.22 & 57.0 & 0.28 & 65.2 & 0.77 & 105 \\
\hline $\mathrm{NO2}, \mathrm{ppm}$ & & 0.02 & 5.14 & 0.03 & 8.93 & 0.04 & 11.9 & 0.06 & 14.4 & 0.07 & 16.5 & 0.19 & 26.5 \\
\hline $\mathrm{N} 2, \%$ & & 1,828 & 62.1 & 2,044 & 62.2 & 2,255 & 62.3 & 2,464 & 62.4 & 2,671 & 62.4 & 4,590 & 62.5 \\
\hline SO2, ppm & & 0.06 & 18.9 & 0.06 & 17.0 & 0.06 & 15.6 & 0.06 & 14.4 & 0.06 & 13.4 & 0.09 & 12.2 \\
\hline $\mathrm{HCl}, \mathrm{ppm}$ & & 0.55 & 187 & 1.08 & 327 & 1.59 & 439 & 2.09 & 530 & 2.59 & 607 & 7.18 & 978 \\
\hline $\mathrm{H} 2 \mathrm{O}, \%$ & & 697 & 23.7 & 772 & 23.5 & 846 & 23.4 & 919 & 23.3 & 991 & 23.2 & 1,676 & 22.8 \\
\hline THC as C & $\mathrm{H} 2, \mathrm{ppm}$ & 0.00 & 0.00 & 0.00 & 0.00 & 0.00 & 0.00 & 0.00 & 0.00 & 0.00 & 0.00 & 0.00 & 0.00 \\
\hline & Total & 2.942 & & 3,285 & & 3,620 & & 3,950 & & 4.278 & & 7,339 & \\
\hline & & & & & & & & & & & & & \\
\hline & & & & & & & & & & & & & \\
\hline Offgas Flow & rate, scfm & & 2,942 & & 3.285 & & 3.620 & & 3,950 & & 4,278 & & 7,339 \\
\hline & $\mathrm{acfm}$ & & 4.234 & & 4,727 & & 5,209 & & 5,684 & & 6,156 & & 10,561 \\
\hline & & & & & & & & & & & & & \\
\hline Offgas moler & cular weight, wt. basis & & 26.5 & & 26.5 & & 26.6 & & 26.6 & & 26.6 & & 26.7 \\
\hline Offgas Flow & rate, $\mathrm{b} / \mathrm{hr}$ & & 12,142 & & 13,572 & & 14,970 & & 16,349 & & 17.717 & & 30,473 \\
\hline Offgas Flow & rate, $\mathrm{kg} / \mathrm{hr}$ & & 5,513 & & 6,162 & & 6,796 & & 7,422 & & 8,043 & & 13,835 \\
\hline & & & & & & & & & & & & & \\
\hline Operating C & onditions & & & & & & & & & & & & \\
\hline & Operating Temperature, F & & 280 & & 280 & & 280 & & 280 & & 280 & & 280 \\
\hline & Operating Temperature, $\mathrm{C}$ & & 138 & & 138 & & 138 & & 138 & & 138 & & 138 \\
\hline & Static pressure, in. $\mathbf{H 2 O}$ & & -10.00 & & -10.00 & & -10.00 & & -10.00 & & -10.00 & & -10.00 \\
\hline & Pulsed jet air, scfm & & 50.0 & & 50.0 & & 50.0 & & 50.0 & & 50.0 & & 50.0 \\
\hline & & & & & & & & & & & & & \\
\hline
\end{tabular}


Table C-1. continued.

\begin{tabular}{|c|c|c|c|c|c|c|c|c|c|c|c|c|c|}
\hline \multirow{2}{*}{\multicolumn{2}{|c|}{ Parameter }} & \multicolumn{2}{|c|}{ NOM-90 } & \multicolumn{2}{|c|}{ NOM-80 } & \multicolumn{2}{|c|}{ NOM-70 } & \multicolumn{2}{|c|}{ NOM-60 } & \multicolumn{2}{|c|}{ NOM-50 } & \multicolumn{2}{|c|}{ NOM-0 } \\
\hline & & $\begin{array}{c}\text { Flowrate } \\
\text { scfm }\end{array}$ & $\begin{array}{l}\text { Comp- } \\
\text { osition }\end{array}$ & $\begin{array}{c}\text { Flowrate } \\
\text { scfm }\end{array}$ & $\begin{array}{l}\text { Comp- } \\
\text { osition }\end{array}$ & $\begin{array}{c}\text { Flowrate } \\
\text { scfm }\end{array}$ & $\begin{array}{l}\text { Comp- } \\
\text { osition }\end{array}$ & $\begin{array}{c}\text { Flowrate } \\
\text { scfm }\end{array}$ & $\begin{array}{l}\text { Comp- } \\
\text { osition }\end{array}$ & $\begin{array}{c}\text { Flowrate } \\
\text { scfm }\end{array}$ & $\begin{array}{l}\text { Comp- } \\
\text { osition }\end{array}$ & $\begin{array}{c}\text { Flowrate } \\
\text { scfm }\end{array}$ & $\begin{array}{l}\text { Comp- } \\
\text { osition }\end{array}$ \\
\hline \multicolumn{14}{|c|}{ SCRUBBER OUTLET } \\
\hline \multicolumn{14}{|l|}{ Composition } \\
\hline $02, \%$ & & 345 & 11.2 & 384 & 11.1 & 423 & 11.1 & 461 & 11.1 & 499 & 11.1 & 852 & 11.0 \\
\hline $\mathrm{CO} 2, \%$ & & 72.2 & 2.34 & 83.1 & 2.41 & 93.8 & 2.46 & 104 & 2.51 & 115 & 2.55 & 213 & 2.75 \\
\hline $\mathrm{Co}, \mathrm{ppm}$ & & 0.01 & 2.80 & 0.01 & 2.80 & 0.01 & 2.80 & 0.01 & 2.80 & 0.01 & 2.80 & 0.02 & 2.81 \\
\hline NO, ppm & & 0.06 & 19.0 & 0.12 & 33.4 & 0.17 & 44.8 & 0.22 & 54.1 & 0.28 & 61.9 & 0.77 & 100 \\
\hline NO2, ppm & & 0.02 & 4.91 & 0.03 & 8.51 & 0.04 & 11.4 & 0.06 & 13.7 & 0.07 & 15.6 & 0.19 & 25.1 \\
\hline $\mathrm{N} 2, \%$ & & 1,828 & 59.3 & 2,044 & 59.3 & 2,255 & 59.3 & 2,464 & 59.3 & 2,671 & 59.3 & 4,590 & 59.1 \\
\hline $\mathrm{SO} 2, \mathrm{ppm}$ & & 0.00 & 0.18 & 0.00 & 0.16 & 0.00 & 0.15 & 0.00 & 0.14 & 0.00 & 0.13 & 0.00 & 0.12 \\
\hline $\mathrm{HCl}, \mathrm{ppm}$ & & 0.01 & 3.58 & 0.02 & 6.24 & 0.03 & 8.35 & 0.04 & 10.1 & 0.05 & 11.5 & 0.14 & 18.5 \\
\hline $\mathrm{H} 2 \mathrm{O}, \%$ & & 835 & $21 \%$ & 935 & 1. & 1,032 & 16 & 1,128 & 像 & 1,223 & X.6. & 2,105 & \% \\
\hline \multicolumn{2}{|c|}{$\mathrm{THC}$ as $\mathrm{CH} 2, \mathrm{ppm}$} & 0.00 & 0.00 & 0.00 & 0.00 & 0.00 & 0.00 & 0.00 & 0.00 & 0.00 & 0.00 & 0.00 & 0.00 \\
\hline & Total & 3,080 & & 3,446 & & 3,804 & & 4,157 & & 4,507 & & $\mathbf{7 , 7 6 2}$ & \\
\hline \multicolumn{2}{|c|}{ Flowrate, scfm } & & 3,080 & & 3,446 & & 3,804 & & 4,157 & & 4,507 & & 7,762 \\
\hline $\mathrm{acfm}$ & & & 3,737 & & 4,181 & & 4,616 & & 5,044 & & 5,469 & & 9,418 \\
\hline \multicolumn{2}{|c|}{ Offgas molecular weight, wt. basis } & & 26.1 & & 26.1 & & 26.1 & & 26.1 & & 26.1 & & 26.2 \\
\hline \multicolumn{2}{|c|}{ Offgas flowrate, ib/hr } & & 12,527 & & 14,021 & & 15,482 & & 16,923 & & 18,352 & & 31,636 \\
\hline \multicolumn{2}{|c|}{ Offgas flowrate, $\mathrm{kg} / \mathrm{hr}$} & & 5.687 & & 6,366 & & 7,029 & & 7,683 & & 8,332 & & 14,363 \\
\hline \multicolumn{2}{|c|}{ Heat capacity of offgas, $\mathrm{J} / \mathrm{mol}^{*} \mathrm{C}$} & & 30.7 & & 30.7 & & 30.7 & & 30.7 & & 30.7 & & 30.7 \\
\hline \multicolumn{2}{|c|}{ Makeup $\mathrm{H} 2 \mathrm{O}$ from evaporation, gpm } & & 0.78 & & 0.91 & & 1.04 & & 1.17 & & 1.30 & & 2.41 \\
\hline \multicolumn{3}{|c|}{ Makeup $\mathrm{H} 20$ from evaporation, $\mathrm{kg} / \mathrm{hr}$} & 176 & & 207 & & 237 & & 266 & & 295 & & 547 \\
\hline & & & & & & & & & & & & & \\
\hline \multicolumn{14}{|c|}{ Operating Conditions } \\
\hline & Outlet Temperature, $\mathbf{F}$ & & 153 & & 153 & & 153 & & 153 & & 153 & & 153 \\
\hline & Outlet Temperature, $\mathrm{C}$ & & 67.2 & & 67.2 & & 67.2 & & 67.2 & & 67.2 & & 67.2 \\
\hline & Static pressure, in. H2O & & -17.00 & & -17.00 & & -17.00 & & -17.00 & & -17.00 & & -17.00 \\
\hline & Scrubbing Efficiency \% & & & & & & & & & & & & \\
\hline & SO2 & . & 99.0 & & 99.0 & & 99.0 & & 99.0 & & 99.0 & & 99.0 \\
\hline & $\mathrm{HCl}$ & & 98.0 & & 98.0 & & 98.0 & & 98.0 & & 98.0 & & 98.0 \\
\hline & & & & & & & & & & & & & \\
\hline Note: & Shaded cells were calculate & ted using a & therative go & -seek functi & that was $b$ & d on chang & the value & the italic & cells until & desired & e was obtai & in the & d cell. \\
\hline & & & & & & & & & & & & & \\
\hline
\end{tabular}


Table C-1. continued.

\begin{tabular}{|c|c|c|c|c|c|c|c|c|c|c|c|c|c|}
\hline \multirow[b]{2}{*}{ Parameter } & & \multicolumn{2}{|c|}{ NOM-90 } & \multicolumn{2}{|c|}{ NOM-80 } & \multicolumn{2}{|c|}{ NOM-70 } & \multicolumn{2}{|c|}{ NOM-60 } & \multicolumn{2}{|c|}{ NOM-50 } & \multicolumn{2}{|c|}{ NOM-0 } \\
\hline & & $\begin{array}{c}\text { Flowrate } \\
\text { scfm }\end{array}$ & $\begin{array}{l}\text { Comp- } \\
\text { osition }\end{array}$ & $\begin{array}{c}\text { Flowrate } \\
\text { scfm }\end{array}$ & $\begin{array}{l}\text { Comp- } \\
\text { osition }\end{array}$ & $\begin{array}{c}\text { Flowrate } \\
\text { scfm }\end{array}$ & $\begin{array}{l}\text { Comp- } \\
\text { osition }\end{array}$ & $\begin{array}{c}\text { Flowrate } \\
\text { scfm }\end{array}$ & $\begin{array}{l}\text { Comp- } \\
\text { osition } \\
\end{array}$ & $\begin{array}{c}\text { Flowrate } \\
\text { scfm }\end{array}$ & $\begin{array}{l}\text { Comp- } \\
\text { osition }\end{array}$ & $\begin{array}{c}\text { Flowrate } \\
\text { scfm }\end{array}$ & $\begin{array}{l}\text { Comp- } \\
\text { osition }\end{array}$ \\
\hline \multicolumn{14}{|c|}{ COOLER/CONDENSER } \\
\hline \multicolumn{14}{|l|}{ Composition } \\
\hline $02, \%$ & & 345 & 14.6 & 384 & 14.6 & 423 & 14.5 & 461 & 14.5 & 499 & 14.5 & 852 & 14.3 \\
\hline $\mathrm{CO} 2, \%$ & & 72.2 & 3.06 & 83.1 & 3.15 & 93.8 & 3.22 & 104 & 3.28 & 115 & 3.32 & 213 & 3.59 \\
\hline $\mathrm{CO}, \mathrm{ppm}$ & & 0.01 & 3.66 & 0.01 & 3.66 & 0.01 & 3.66 & 0.01 & 3.65 & 0.01 & 3.65 & 0.02 & 3.67 \\
\hline NO, ppm & & 0.06 & 24.8 & 0.12 & 43.6 & 0.17 & 58.5 & 0.22 & 70.7 & 0.28 & 80.8 & 0.77 & 130 \\
\hline \multicolumn{2}{|l|}{ NO2, ppm } & 0.02 & 6.41 & 0.03 & 11.1 & 0.04 & 14.8 & 0.06 & 17.9 & 0.07 & 20.4 & 0.19 & 32.7 \\
\hline N2, \% & & 1.828 & 77.5 & 2.044 & 77.4 & 2,255 & 77.4 & 2,464 & 77.4 & 2,671 & 77.4 & 4,590 & 77.2 \\
\hline $\mathrm{SO} 2, \mathrm{ppm}$ & & $0(0)$ & 0.24 & 0.00 & 0.21 & 0.00 & 0.19 & 0.00 & 0.18 & 0.00 & 0.17 & 0.00 & 0.15 \\
\hline $\mathrm{HCl}_{1} \mathrm{ppm}$ & & 0.01 & 4.67 & 0.02 & 8.15 & 0.03 & 10.9 & 0.04 & 13.2 & 0.05 & 15.0 & 0.14 & 24.2 \\
\hline H2O. \% & & 114 & 4.84 & 128 & 4.84 & 141 & 884 & 154 & 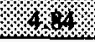 & 167 & 186 & 288 & \% \\
\hline \multicolumn{2}{|c|}{ THC as CH2. ppm } & 000 & 0.00 & 0.00 & 0.00 & 0.00 & 0.00 & 0.00 & 0.00 & 0.00 & 0.00 & 0.00 & 0.00 \\
\hline & Toual & 2.359 & & 2.639 & & 2.913 & & 3,184 & & 3,452 & $\therefore$ & 5,944 & \\
\hline \multicolumn{2}{|c|}{ Flowrate, scim } & & 2.159 & & 2.639 & & 2,913 & & 3,184 & & 3,452 & & 5,944 \\
\hline acfm & & & 2.581 & & 2.888 & & 3,188 & & 3,484 & & 3,777 & & 6,504 \\
\hline \multicolumn{2}{|c|}{$\mathrm{H} 20$ cond. from gas, $\mathrm{kg} / \mathrm{hr}$} & 919 & $\ldots$ & 1.028 & & 1,135 & & 1.240 & & 1,344 & & 2,315 & \\
\hline \multicolumn{2}{|c|}{$\mathrm{H} 2 \mathrm{O}$ cond. from gas. $\mathrm{gpm}$} & 4.04 & & 4.52 & & 4.99 & & 5.46 & & 5.92 & & 10.2 & \\
\hline \multicolumn{2}{|c|}{ Offgas molecular weight, wh basis } & & 28.6 & & 28.6 & & 28.6 & & 28.6 & & 28.6 & & 28.7 \\
\hline \multicolumn{2}{|c|}{ Offgas flowrate. Ib/hr } & & 10,505 & & 11.759 & & 12.985 & & 14,194 & & 15,393 & & 26,541 \\
\hline \multicolumn{2}{|c|}{ Offgas flowrate, $\mathrm{kg} / \mathrm{hr}$} & & 4.769 & & 5,339 & & 5,895 & & 6,444 & & 6,988 & & 12,050 \\
\hline & & & & & & & & & & & & & \\
\hline \multicolumn{14}{|c|}{ Operating Conditions } \\
\hline & Outlet Temperature, F & & 90.0 & & 90.0 & & 90.0 & & 90.0 & & 90.0 & & 90.0 \\
\hline & Outlet Temperature, C & & 32.2 & & 32.2 & & 32.2 & & 32.2 & & 32.2 & & 32.2 \\
\hline & Static pressure, in. H2O & & -19.00 & & -19.00 & & -19.00 & & -19.00 & & -19.00 & & -19.00 \\
\hline & Condenser water, gal/min & & 8.77 & & 9.8 & & 10.8 & & 11.8 & & 12.8 & & 22.1 \\
\hline \multirow[t]{3}{*}{ Notes: } & 1. Assumes cond. $\mathrm{H} 2 \mathrm{O} \mathrm{T}$ & $T$ of $60 \mathrm{~F}$, & d approacl & of $23 \mathrm{~F}$ & & & & & & & & & \\
\hline & 2. Shaded cells were calct & culated usir & an iterative & oal-seck fu & tion that $w$ & based on $\mathrm{c}$ & jing the va & $s$ in the it & ed cells u & II the desir & ralue was o & tained in the & haded cell. \\
\hline & & & & & & & & & & & & & \\
\hline
\end{tabular}


Table C-1. continued.

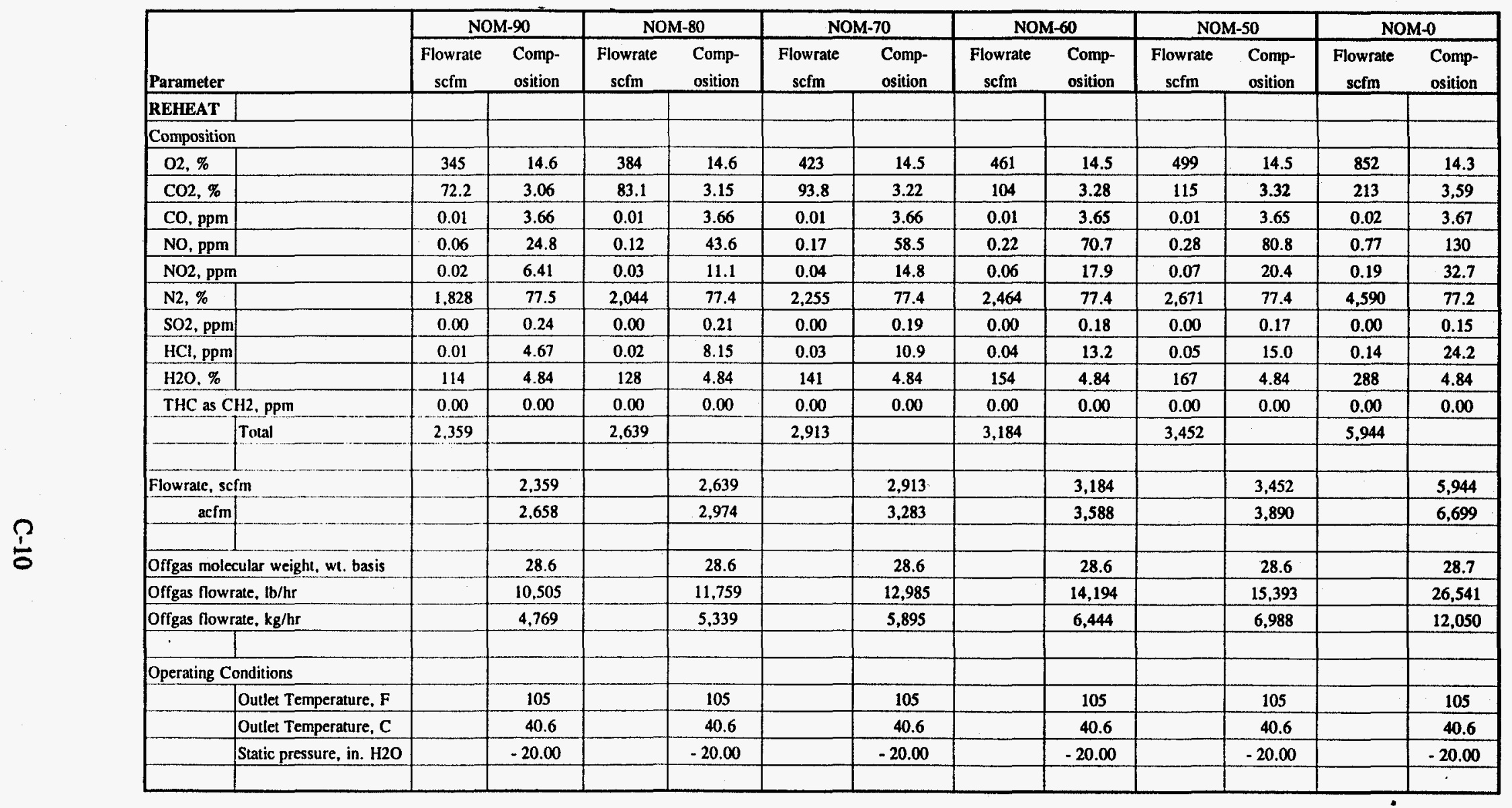


Table C-1. continued.

\begin{tabular}{|c|c|c|c|c|c|c|c|c|c|c|c|c|c|}
\hline \multirow{2}{*}{ Parameter } & & \multicolumn{2}{|c|}{ NOM-90 } & \multicolumn{2}{|c|}{ NOM-80 } & \multicolumn{2}{|c|}{ NOM-70 } & \multicolumn{2}{|c|}{ NOM-60 } & \multicolumn{2}{|c|}{ NOM-50 } & \multicolumn{2}{|c|}{ NOM-0 } \\
\hline & & $\begin{array}{c}\text { Flowrate } \\
\text { scfm }\end{array}$ & $\begin{array}{l}\text { Comp- } \\
\text { osition }\end{array}$ & $\begin{array}{c}\text { Flowrate } \\
\text { scfm }\end{array}$ & $\begin{array}{l}\text { Comp- } \\
\text { osition }\end{array}$ & $\begin{array}{c}\text { Flowrate } \\
\text { scfm }\end{array}$ & $\begin{array}{l}\text { Comp- } \\
\text { osition }\end{array}$ & $\begin{array}{c}\text { Flowrate } \\
\text { scfm }\end{array}$ & $\begin{array}{l}\text { Comp- } \\
\text { osition }\end{array}$ & $\begin{array}{c}\text { Flowrate } \\
\text { scfm }\end{array}$ & $\begin{array}{l}\text { Comp- } \\
\text { osition }\end{array}$ & $\begin{array}{c}\text { Flowrate } \\
\text { sctm }\end{array}$ & $\begin{array}{l}\text { Comp- } \\
\text { osition }\end{array}$ \\
\hline \multicolumn{14}{|c|}{ INDUCED DRAFT FAN } \\
\hline \multicolumn{14}{|l|}{ Composition } \\
\hline $02, \%$ & & 345 & 14.6 & 384 & 14.6 & 423 & 14.5 & 461 & 14.5 & 499 & 14.5 & 852 & 14.3 \\
\hline $\mathrm{CO} 2 \%$ & & 72.2 & 3.06 & 83.1 & 3.15 & 93.8 & 3.22 & 104 & 3.28 & 115 & 3.32 & 213 & 3.59 \\
\hline $\mathrm{co}, \mathrm{ppm}$ & & 0.01 & 3.66 & 0.01 & 3.66 & 0.01 & 3.66 & 0.01 & 3.65 & 0.01 & 3.65 & 0.02 & 3.67 \\
\hline NO, ppm & & 0.06 & 24.8 & 0.12 & 43.6 & 0.17 & 58.5 & 0.22 & 70.7 & 0.28 & 80.8 & 0.77 & 130 \\
\hline $\mathrm{NO} 2, \mathrm{ppm}$ & & 0.02 & 6.41 & 0.03 & 11.1 & 0.04 & 14.8 & 0.06 & 17.9 & 0.07 & 20.4 & 0.19 & 32.7 \\
\hline $\mathrm{N} 2, \%$ & & 1,828 & 77.5 & 2,044 & 77.4 & 2,255 & 77.4 & 2,464 & 77.4 & 2,671 & 77.4 & 4,590 & 77.2 \\
\hline SO2,ppm & & 0.00 & 0.24 & 0.00 & 0.21 & 0.00 & 0.19 & 0.00 & 0.18 & 0.00 & 0.17 & 0.00 & 0.15 \\
\hline $\mathrm{HCl}, \mathrm{ppm}$ & & 0.01 & 4.67 & 0.02 & 8.15 & 0.03 & 10.9 & 0.04 & 13.2 & 0.05 & 15.0 & 0.14 & 24.2 \\
\hline $\mathrm{H} 2 \mathrm{O}, \%$ & & 114 & 4.84 & 128 & 4.84 & 141 & 4.84 & 154 & 4.84 & 167 & 4.84 & 288 & 4.84 \\
\hline \multicolumn{2}{|c|}{ THC as $\mathrm{CH} 2$, ppm } & 0.00 & 0.00 & 0.00 & 0.00 & 0.00 & 0.00 & 0.00 & 0.00 & 0.00 & 0.00 & 0.00 & 0.00 \\
\hline & Total & 2,359 & & 2,639 & & 2,913 & & 3,184 & & 3,452 & & 5,944 & \\
\hline & & & & & & & & & & & & & \\
\hline \multicolumn{2}{|c|}{ Flowrate, scfm } & & 2,359 & & 2,639 & & 2,913 & & 3,184 & & 3.452 & & 5,944 \\
\hline acfm & & & 2,572 & & 2,878 & & 3,177 & & 3,472 & & 3.764 & & 6,482 \\
\hline & & & 28.6 & & 28.6 & & 28.6 & & 28.6 & & 28.6 & & 28.7 \\
\hline \multicolumn{2}{|c|}{ Offgas molecular weight, wt. basis } & & 10,505 & & 11,759 & & 12,985 & & 14,194 & & 15,393 & & 26,541 \\
\hline \multicolumn{2}{|c|}{\begin{tabular}{|l|} 
Offgas flowrate, $\mathrm{lb} / \mathrm{hr}$ \\
Offgas flowrate, $\mathrm{kg} / \mathrm{hr}$
\end{tabular}} & & 4,769 & & 5,339 & & 5,895 & & 6,444 & & 6,988 & & 12,050 \\
\hline & & & & & & & & & & & & & \\
\hline \multicolumn{14}{|c|}{ Operating Conditions } \\
\hline & Outlet Temperature, $\mathrm{F}$ & & 115 & & 115 & & 115 & & 115 & & 115 & & 115 \\
\hline & Outlet Temperature, C & & 46.1 & & 46.1 & & 46.1 & & 46.1 & & 46.1 & & 46.1 \\
\hline & & & & & & & & & & & & & \\
\hline
\end{tabular}




\section{Appendix D}

Estimated Elemental Compositions of the Surrogate Mixture Components

D-1 


$$
\text { D-2 }
$$


Table D-1. Estimated elemental compositions of the surrogate additives used to prepare the surrogate waste mixtures.

\begin{tabular}{|c|c|}
\hline Additive & Comp. \\
\hline Lime & \\
\hline $\mathrm{CaO}$ & 96.0 \\
\hline $\mathrm{SiO} 2$ & 1.50 \\
\hline $\mathrm{CO} 2$ & 0.80 \\
\hline $\mathrm{Fe} 2 \mathrm{O} 3$ & 0.12 \\
\hline Al2O3 & 0.45 \\
\hline $\mathrm{MgO}$ & 0.50 \\
\hline $\mathrm{SO} 3$ & 0.20 \\
\hline $\mathrm{MnO} 2$ & 0.05 \\
\hline $\mathrm{TiO} 2$ & 0.05 \\
\hline $\mathrm{Na2O}$ & 0.03 \\
\hline $\mathrm{P} 2 \mathrm{O5}$ & 0.02 \\
\hline $\mathbf{F}$ & 0.03 \\
\hline $\mathbf{P b}$ & 0.00 \\
\hline As & 0.00 \\
\hline Carbon steel & \\
\hline $\mathrm{Fe}$ & 99.8 \\
\hline $\mathrm{C}$ & 0.20 \\
\hline Stainless steel & \\
\hline $\mathrm{Fe}$ & 74.0 \\
\hline $\mathrm{Cr}$ & 18.0 \\
\hline $\mathrm{Ni}$ & 8.00 \\
\hline Aluminum & 100 \\
\hline Zirconium & \\
\hline $\mathrm{Zr}$ & 93.0 \\
\hline Mo & 4.70 \\
\hline $\mathrm{Si}$ & 1.50 \\
\hline $\mathrm{Fe}$ & 0.40 \\
\hline $\mathrm{Cr}$ & 0.30 \\
\hline Hf & 0.10 \\
\hline $\mathrm{Cu}$ & 0.09 \\
\hline Mn & 0.09 \\
\hline Copper & 100 \\
\hline Lead & 100 \\
\hline Polyethylene & \\
\hline C & 85.6 \\
\hline $\mathbf{H}$ & 14.4 \\
\hline \multicolumn{2}{|c|}{ Wood pellets (paper) } \\
\hline C & 34.5 \\
\hline H & 6.54 \\
\hline 0 & 48.9 \\
\hline $\mathbf{N}$ & 0.09 \\
\hline Water & 10.0 \\
\hline
\end{tabular}

\begin{tabular}{|c|c|}
\hline Additive & Comp. \\
\hline \multicolumn{2}{|l|}{ Rubber } \\
\hline C & 54.3 \\
\hline $\mathbf{H}$ & 5.70 \\
\hline $\mathrm{Cl}$ & 40.0 \\
\hline \multicolumn{2}{|l|}{ Oil Dri sorbent } \\
\hline $\mathrm{SiO} 2$ & 89.2 \\
\hline $\mathrm{A} 12 \mathrm{O} 3$ & 4.00 \\
\hline $\mathrm{Fe} 2 \mathrm{O} 3$ & 1.50 \\
\hline $\mathrm{CaO}$ & 0.50 \\
\hline $\mathrm{MgO}$ & 0.30 \\
\hline $\mathrm{Na} 2 \mathrm{O}$ & 0.25 \\
\hline $\mathrm{K} 2 \mathrm{O}$ & 0.25 \\
\hline Water & 4.00 \\
\hline \multicolumn{2}{|c|}{ Wood pellets (cloth) } \\
\hline C & 34.5 \\
\hline $\mathbf{H}$ & 6.54 \\
\hline 0 & 48.9 \\
\hline $\mathbf{N}$ & 0.09 \\
\hline Water & 10.0 \\
\hline \multicolumn{2}{|c|}{ Texaco Regal Oil } \\
\hline C & 85.6 \\
\hline H & 12.0 \\
\hline$S$ & 0.35 \\
\hline $\mathbf{0}$ & 0.60 \\
\hline $\mathbf{N}$ & 0.50 \\
\hline \multicolumn{2}{|l|}{ Hydraulic oil } \\
\hline c & 85.6 \\
\hline H & 12.0 \\
\hline $\mathbf{S}$ & 0.35 \\
\hline o & 0.60 \\
\hline $\mathbf{N}$ & 0.50 \\
\hline \multicolumn{2}{|c|}{ Wheel beanng grease } \\
\hline c & 85.6 \\
\hline H & 12.0 \\
\hline $\mathbf{S}$ & 0.35 \\
\hline 0 & 0.60 \\
\hline $\mathbf{N}$ & 0.50 \\
\hline \multicolumn{2}{|l|}{ PVC } \\
\hline c & 38.4 \\
\hline $\mathrm{H}$ & 4.85 \\
\hline Cl & 56.7 \\
\hline
\end{tabular}

\begin{tabular}{|c|c|}
\hline Additive & Comp. \\
\hline $\mathrm{NaNO} 3$ & 100 \\
\hline KNO3 & 100 \\
\hline $\mathrm{NaCl}$ & 100 \\
\hline $\mathrm{Na} 2 \mathrm{SO}_{4}$ & 100 \\
\hline $\mathrm{NaOH}$ & 100 \\
\hline Water & 100 \\
\hline Al(OH)3 & 100 \\
\hline $\mathrm{Fe}(\mathrm{OH}) 3$ & 100 \\
\hline $\mathrm{Ca}(\mathrm{OH}) 2$ & 100 \\
\hline $\mathrm{Mg}(\mathrm{OH}) 2$ & 100 \\
\hline $\mathrm{KOH}$ & 100 \\
\hline $\mathrm{H} 2 \mathrm{O}$ & 100 \\
\hline \multicolumn{2}{|c|}{ Portland Cement } \\
\hline $\mathrm{SiO} 2$ & 21.0 \\
\hline $\mathrm{Al} 2 \mathrm{O} 3$ & 5.80 \\
\hline $\mathrm{Fe} 2 \mathrm{O} 3$ & 2.40 \\
\hline $\mathrm{TiO} 2$ & 0.42 \\
\hline $\mathrm{Mn} 2 \mathrm{O} 3$ & 0.07 \\
\hline P2OS & 0.20 \\
\hline $\mathrm{SO} 3$ & 2.52 \\
\hline $\mathrm{CaO}$ & 64.0 \\
\hline $\mathrm{MgO}$ & 1.24 \\
\hline $\mathrm{Na} 2 \mathrm{O}$ & 0.25 \\
\hline $\mathrm{K} 2 \mathrm{O}$ & 0.61 \\
\hline Water & 1.49 \\
\hline \multicolumn{2}{|c|}{ Wood pellets (wood) } \\
\hline c & 34.5 \\
\hline $\mathbf{H}$ & 6.54 \\
\hline 0 & 48.9 \\
\hline $\mathbf{N}$ & 0.09 \\
\hline Water & 10.0 \\
\hline Microcel E & \\
\hline $\mathrm{SiO} 2$ & 56.0 \\
\hline $\mathrm{Al} 203$ & 3.80 \\
\hline $\mathrm{Fe} 2 \mathrm{O} 3$ & 1.00 \\
\hline $\mathrm{CaO}$ & 26.0 \\
\hline $\mathrm{MgO}$ & 0.70 \\
\hline $\mathrm{Na} 2 \mathrm{O}$ & 0.60 \\
\hline $\mathbf{K} 2 \mathrm{O}$ & 0.60 \\
\hline Water & 10.0 \\
\hline
\end{tabular}

\begin{tabular}{|c|c|}
\hline Additive & Comp. \\
\hline & \\
Soil & \\
Ag2O & 0.00 \\
Al2O3 & 10.41 \\
As2O & 0.00 \\
BaO & 0.01 \\
CaCO3 & 13.00 \\
CaO & 0.11 \\
CdO & 0.00 \\
CeO2 & 0.01 \\
Cr2O3 & 0.03 \\
Cs2O & 0.01 \\
CuO & 0.01 \\
Fe3O4 & 4.23 \\
HgO & 0.00 \\
K2O & 2.42 \\
MgO & 2.27 \\
Na2O & 1.28 \\
NiO & 0.00 \\
PO4 & 0.29 \\
PbO & 0.00 \\
SO4 & 0.04 \\
NeO & 0.00 \\
SiO & 0.19 \\
SiO2 & 57.76 \\
TiO2 & 0.43 \\
ZnO & 0.01 \\
ZrO2 & 0.01 \\
Cl & 0.00 \\
Mill scale & 10.10 \\
\hline & 0.21 \\
\hline
\end{tabular}




\section{Appendix $E$}

Reconciliation of the Test Plan to the BWID Technology Test Plan Guidance Document 



\section{Appendix E}

\section{Reconciliation of the Test Plan to the BWID Technology Test Plan Guidance Document}

This test plan was prepared according to the Buried Waste Integrated Demonstration Technology Test Plan (EGG-WTD-9800, Rev. 1), with some deviations that were pertinent to this specific test program. This test plan has been written as a guide for those LITCO and U.S. Bureau of Mines personnel to perform the test program and meet the objectives specified in the Technical Task Plan. The test plan was organized to reduce redundancy, maintain this focus, and minimize distraction during day-to-day use of this test plan during the test program. Several of the sections specified in the general guidance document are not relevant to this program and were excluded. Some other sections have been reorganized so the test plan is more directly focused on the test program.

For completeness, the contents of the test plan and the recommended sections in the guidance document are reconciled in Table E-1. 
Table E-1. Reconciliation of the test plan to the BWID technology test plan guidance document. Section of the guidance document (EGGWTD-9800, Rev.1, App. A)

Location where found in the test plan

ABSTRACT

ACRONYMS

1. INTRODUCTION

1.1 Technology Description and Background

1.2 Scope of Test

1.3 Objective of Test

1.4 Technology Agreement

1.5 The customer need that the technology is satisfying

2. ORGANIZATION AND RESPONSIBILITIES

2.1 Organizational Responsibilities chart

2.2 Responsibilities of performing organization

2.3 Personnel Support Requirements

2.4 Test Personnel Qualifications List

3. DESCRIPTION OF TEST

3.1 Key Input and Output Parameters

3.2 Test Matrix

3.3 Test Methods and Uncertainties

3.4 Test Procedures

3.5 Illustrations

3.6 Contingency Plans
ABSTRACT

ACRONYMS

1. INTRODUCTION

1.1 Technology Description and Background

1.2 Scope

1.3 Objectives

1.4 Technology Agreement

Customer needs are more completely defined in the Technical Task Plan (TTP), and will be satisfied by completion of the test objectives defined in the TTP and in Section 1.3.

\section{ORGANIZATION AND RESPONSIBILITIES}

The chart is redundant with the text.

Section 2.1

Section 2.1

Section 2.1, to the extent necessary.

3. DESCRIPTION OF TEST - This section was organized to describe the facility first, as necessary, to enable the reader to understand the rest of the test program.

3.2

3.2, 3.2.2, 5, 7

$3.2,5,7$

Scattered throughout sections as applicable to the discussion.

3.2 .3 
3.7 Performance Objectives

3.8 How Testing Meets Customer Need

4. SEQUENCE OF ACTIVITIES

5. SAMPLING AND DATA

5.1 Data Applicability to CERCLA Criteria

5.2 Data Collection Techniques

5.3 Sampling and Data Collection Procedures

5.4 Sampling and Analysis Requirements

6. DOCUMENT CONTROL

6.1 Data Documentation, Control, and Storage

6.2 Test Plan Modifications

6.3 Document Control for Other Documents

7. ANALYTICAL METHODS

8. DATA REDUCTION, VALIDATION, AND VERIFICATION

8.1 Data Reduction Scheme

8.2 Data Validation

8.3 Data Validation Responsibility
Not applicable

Customer needs are more completely defined in the Technical Task Plan (TIP) and will be satisfied by completion of the test objectives defined in the TTP and in Section 1.3.

\section{SEQUENCE OF ACTIVITIES \\ 5. MEASUREMENTS AND SAMPLE COLLECTION}

Not applicable at this point in the demonstration program.

Throughout Sections 3.2 and 5. Standard procedures are not described in detail but will follow the referenced procedures.

Throughout sections 3.2 and 5. Standard procedures are not described in detail but will follow the referenced procedures.

Section 5

6. DOCUMENT CONTROL

6.1 Data Documentation, Control, and Storage

6.2 Test Plan Change Control

6.3 Document Control for Other Documents

7. ANALYTICAL METHODS. Standard procedures are not described in detail but will follow the referenced procedures.

8. DATA REDUCTION, VALIDATION, AND VERIFICATION

8.1 Data Reduction Scheme and in procedures referenced in Sections 5.3.1, 5.3.2, 5.3.3, and 7 .

8.2 Data Validation and in procedures referenced in Sections 5.3.1, 5.3.2, 5.3.3, and 7 .

8.3 Data Validation Responsibilities 


\subsection{Acceptance Criteria}

9. QUALTTY ASSURANCE

9.1 Quality Level

9.2 Quality Control Methods

9.3 Routine Procedures for Assessing Precision, Accuracy and Completeness

9.4 Change Authorization

9.5 Internal Audit/Surveillance System

10. EQUIPMENT AND INSTRUMENTS

10.1 Equipment List

10.2 Equipment Range, Accuracy and Tolerance

10.3 Calibration and Standardization Procedures

10.4 Preventive Maintenance Schedule

10.5 Sensitivity to the Environment

11. SUPPLIES, UTILITIES AND

FACILITIES

12. HEALTH AND SAFETY

13. RESIDUALS MANAGEMENT

14. REFERENCES

15. APPENDICES
8.4 Acceptance Criteria for Test Data, especially Tables 8-1, 8-2, and 8-3.

9. QUALTTY ASSURANCE

9.1 Quality Level

9.2 Quality Control Methods

9.3 Routine Procedures, to the extent needed for this test plan.

Section 6.2

Section 9.4

Sections 3, 5, 7, and 10

Sections 5, 7, and 10.1 (to the degree necessary for this test plan).

Sections $5,7,8$, and 10.2 (to the degree necessary for this test plan).

Sections $5,7,8$, and 10.3 (to the degree necessary for this test plan).

Described in procedures referenced in Sections 5 and 7

Described in procedures referenced in Sections 5 and 7

11. SUPPLIES, UTILITIES, AND FACILITIES, and as described in procedures referenced in Sections 5 and 7

12. HEALTH AND SAFETY

13. RESIDUALS MANAGEMENT

14. REFERENCES

15. APPENDICES 